\title{
Synthesis of \\ Pyrido[2,3-e]pyrrolo[1,2-a]pyrazine Derivatives via Tandem Iminium Cyclization and Smiles Rearrangement
}

\author{
Jinbao Xiang, Hongxiang Xie, Dongsheng Wen, Qun Dang* and Xu Bai * \\ The Center for Combinatorial Chemistry and Drug Discovery, Jilin University, 75 \\ Haiwai St., Changchun, Jilin 130012, P. R. China \\ xbai@jlu.edu.cn or qdang@jlu.edu.cn
}

Table of Contents

$\begin{array}{ll}\text { Experimental Section-General Considerations } & \text { S2 }\end{array}$

Experimental Details and Spectroscopic Data $\quad$ S2

$\begin{array}{ll}\text { Figure Legends } & \text { S10 }\end{array}$

$\begin{array}{ll}\text { Spectra } & \text { S14 }\end{array}$

Tel: +86-431-85188955; Fax: +86-431-85188900; E-mail: xbai@jlu.edu.cn or qdang@jlu.edu.cn 


\section{General Considerations.}

Acetonitrile $\left(\mathrm{CH}_{3} \mathrm{CN}\right)$ was dried with $\mathrm{CaH}_{2}$ and distilled. Dichloromethane (DCM) was dried with $\mathrm{P}_{2} \mathrm{O}_{5}$ and distilled. All other commercial reagents were used as received without purification. Melting points were uncorrected. Mass spectra and HPLC data were recorded on a LC/MS system with ELSD. The ${ }^{1} \mathrm{H}$ and ${ }^{13} \mathrm{C}$ NMR data were obtained on a $300 \mathrm{MHz}$ NMR spectrometer with TMS as the internal standard and $\mathrm{CDCl}_{3}$ as the solvent unless otherwise stated. Multiplicities are indicated as the following: s, singlet; $\mathrm{d}$, doublet; $\mathrm{t}$, triplet; $\mathrm{q}$, quartet; $\mathrm{m}$, multiplet; dd, doubled doublet; br, broad. Coupling constants ( $J$ values) are quoted in Hertz.

2-Chloro-3-(1H-pyrrol-1-yl)pyridine 5: 2,5-Dimethoxytetrahydrofuran (17.1 mL, $0.132 \mathrm{mmol}$ ) was added to the stirred solution of 3-amino-2-chloropyridine 4 (17.0 g, $0.132 \mathrm{~mol})$ in acetic acid $(100 \mathrm{~mL})$. The resulting solution was stirred for $1.5 \mathrm{~h}$ at reflux. Then acetic acid was evaporated in vacuo to give the crude product. Purification by flash column chromatography (Petroleum ether/DCM 1:1, v/v) afforded $21.7 \mathrm{~g}(92 \%)$ of 5 as a white solid. mp: $32-33{ }^{\circ} \mathrm{C} .{ }^{1} \mathrm{H}$ NMR $\delta 8.40(\mathrm{~d}, 1 \mathrm{H}, J=$ 4.5), $7.68(\mathrm{~d}, 1 \mathrm{H}, J=7.5), 7.38-7.34(\mathrm{~m}, 1 \mathrm{H}), 6.94(\mathrm{~s}, 2 \mathrm{H}), 6.38(\mathrm{~s}, 2 \mathrm{H})$; MS (ESI): $m / z 179.0\left[\mathrm{M}+\mathrm{H}^{+}\right]$.

2-Hydroxyethoxy-3-pyrrol-1-yl-pyridine 6: To a stirred solution of 2-chloro-3-(1H-pyrrol-1-yl)pyridine 5 (4.55 g, $25.5 \mathrm{mmol})$ and ethyleneglycol (14.0 $\mathrm{mL}, 255 \mathrm{mmol})$ in DMSO (40 mL) was added $\mathrm{KOH}(3.43 \mathrm{~g}, 61.2 \mathrm{mmol})$. After stirring for $12 \mathrm{~h}$ at $50{ }^{\circ} \mathrm{C}$, water $(100 \mathrm{~mL})$ was added, and the reaction mixture was extracted with EtOAc $(5 \times 50 \mathrm{~mL})$. The combined organic layer was washed with 
water $(150 \mathrm{~mL})$. The water layer was extracted with EtOAc $(100 \mathrm{~mL})$. The combined EtOAc layer was dried over $\mathrm{MgSO}_{4}$ and concentrated in vacuo. The residue was purified by flash column chromatography (Petroleum ether/EtOAc 4:1, v/v) afforded $4.89 \mathrm{~g}(94 \%)$ of 6 as a white solid. mp: $26-27{ }^{\circ} \mathrm{C} .{ }^{1} \mathrm{H}$ NMR $\delta 8.05(\mathrm{~d}, 1 \mathrm{H}, J=5.1)$, $7.60(\mathrm{~d}, 1 \mathrm{H}, J=9.6), 7.06-6.99(\mathrm{~m}, 3 \mathrm{H}), 6.34(\mathrm{~s}, 2 \mathrm{H}), 4.55(\mathrm{t}, 2 \mathrm{H}, J=4.5), 3.98-3.93$ $(\mathrm{m}, 2 \mathrm{H}), 3.57(\mathrm{t}, 1 \mathrm{H}, J=6.0) ;{ }^{13} \mathrm{C} \mathrm{NMR} \delta 156.5,143.5,132.7,125.2,121.6,117.5$, 109.7, 69.1, 61.8; MS (ESI): $m / z, 205.1\left[\mathrm{M}+\mathrm{H}^{+}\right]$.

2-(3-(1H-pyrrol-1-yl)pyridin-2-yloxy)acetaldehyde 1: A solution of DMSO (2.02 $\mathrm{mL}, 5.88 \mathrm{mmol})$ in $\mathrm{CH}_{2} \mathrm{Cl}_{2}(6 \mathrm{~mL})$ was added dropwise to a stirred solution of oxalyl chloride $(0.900 \mathrm{~mL}, 11.8 \mathrm{mmol})$ in $\mathrm{CH}_{2} \mathrm{Cl}_{2}(12 \mathrm{~mL})$ at $-78^{\circ} \mathrm{C}$ under $\mathrm{N}_{2}$. After $30 \mathrm{~min}$, a solution of alcohol $6(1.20 \mathrm{~g}, 5.88 \mathrm{mmol})$ in $\mathrm{CH}_{2} \mathrm{Cl}_{2}(6 \mathrm{~mL})$ was added dropwise over $35 \mathrm{~min}$. The resulting solution was stirred for $2 \mathrm{~h}$ at $-70{ }^{\circ} \mathrm{C}$ and for $1.5 \mathrm{~h}$ at -55 to $-45^{\circ} \mathrm{C} . \mathrm{Et}_{3} \mathrm{~N}(10 \mathrm{~mL})$ was added dropwise at $-60{ }^{\circ} \mathrm{C}$. After stirring for an additional 10 min, the reaction mixture was allowed to warm to room temperature. The resulting solution was stirred for $15 \mathrm{~min}$ at room temperature, diluted with $\mathrm{CH}_{2} \mathrm{Cl}_{2}(30 \mathrm{~mL})$, washed with $1 \% \mathrm{HCl}, \mathrm{NaHCO}_{3}(\mathrm{aq})$ and water, dried over $\mathrm{MgSO}_{4}$ and concentrated in vacuo to give the crude aldehyde 1 . The residue was purified by flash column chromatography (Petroleum ether/EtOAc 6:1, v/v) afforded $0.998 \mathrm{~g}(84 \%)$ of $\mathbf{1}$ as a white solid. mp: $21-23{ }^{\circ} \mathrm{C} .{ }^{1} \mathrm{H}$ NMR $\delta 9.75(\mathrm{~s}, 1 \mathrm{H}), 8.01(\mathrm{~d}, 1 \mathrm{H}, J=3.6), 7.63(\mathrm{~d}, 1 \mathrm{H}$, $J=7.8), 7.11(\mathrm{t}, 2 \mathrm{H}, J=2.4), 7.06-6.99(\mathrm{~m}, 1 \mathrm{H}), 6.37(\mathrm{t}, 2 \mathrm{H}, J=2.1), 4.94(\mathrm{~s}, 2 \mathrm{H})$.

TFA-catalyzed reactions for the synthesis of pyrido[2,3-e]pyrrolo[1,2-a] pyrazine 3: The solution of aldehyde $1(101 \mathrm{mg}, 0.5 \mathrm{mmol})$ in $\mathrm{CH}_{3} \mathrm{CN}(10 \mathrm{~mL})$ was 
added dropwise into the solution of an aromatic amine $(0.75 \mathrm{mmol})$ and TFA ( $4 \mu \mathrm{L}$, $0.05 \mathrm{mmol})$ in $\mathrm{CH}_{3} \mathrm{CN}(10 \mathrm{~mL})$ at $80{ }^{\circ} \mathrm{C}$ over 2.5 or $3.0 \mathrm{~h}$. The resulting solution was stirred for the corresponding time at reflux. The solvent was removed in vacuo to give the crude product. Purification by flash chromatography (Petroleum ether/EtOAc = 4:1, v/v) afforded the desired products.

\section{5,6-Dihydro-6-hydroxymethyl-5-(2-methoxylphenyl)pyrido[2,3-e]pyrrolo[1,2-a]p}

yrazine 3a: $46 \%$. mp: $180-181{ }^{\circ} \mathrm{C} .{ }^{1} \mathrm{H}$ NMR (DMSO- $\left.d 6\right) \delta 7.81(\mathrm{~d}, 1 \mathrm{H}, J=7.5), 7.63$ $(\mathrm{d}, 1 \mathrm{H}, J=4.5), 7.52(\mathrm{~s}, 1 \mathrm{H}), 7.47(\mathrm{~d}, 1 \mathrm{H}, J=7.5), 7.31(\mathrm{t}, 1 \mathrm{H}, J=7.5), 7.11(\mathrm{~d}, 1 \mathrm{H}, J$ = 8.1), $7.01(\mathrm{t}, 1 \mathrm{H}, J=7.5), 6.68-6.64(\mathrm{~m}, 1 \mathrm{H}), 6.33(\mathrm{~s}, 1 \mathrm{H}), 6.06(\mathrm{~s}, 1 \mathrm{H}), 4.83(\mathrm{br}$, 2H), $3.68(\mathrm{~s}, 3 \mathrm{H}), 3.51(\mathrm{~d}, 2 \mathrm{H}, J=4.5) ;{ }^{13} \mathrm{C}$ NMR (DMSO-d6) $\delta 155.3,147.2,142.2$, $132.1,130.6,128.0,125.9,120.3,120.1,119.6,114.5,113.2,112.5,111.4,105.2$ 63.6, 58.6, 55.5; MS (ESI): $m / z, 308.2\left[\mathrm{M}+\mathrm{H}^{+}\right]$.

\section{5,6-Dihydro-6-hydroxymethyl-5-(4-methoxylphenyl)pyrido[2,3-e $]$ pyrrolo[1,2-a]p} yrazine 3b: $62 \%$. mp: $67-68{ }^{\circ} \mathrm{C} .{ }^{1} \mathrm{H}$ NMR $\delta 7.88(\mathrm{~d}, 1 \mathrm{H}, J=4.5), 7.51(\mathrm{~d}, 1 \mathrm{H}, J=$ 8.1), $7.28(\mathrm{~d}, 2 \mathrm{H}, J=8.1), 7.20(\mathrm{~s}, 1 \mathrm{H}), 6.93(\mathrm{~d}, 2 \mathrm{H}, J=9.0), 6.74-6.70(\mathrm{~s}, 1 \mathrm{H}), 6.41$ $(\mathrm{m}, 1 \mathrm{H}), 6.09(\mathrm{~s}, 1 \mathrm{H}), 5.01(\mathrm{t}, 1 \mathrm{H}, J=5.1), 3.81(\mathrm{~s}, 3 \mathrm{H}), 3.81-3.62(\mathrm{~m}, 2 \mathrm{H}), 1.92(\mathrm{br}$, $1 \mathrm{H}) ;{ }^{13} \mathrm{C}$ NMR $\delta 157.1,146.9,143.3,136.7,127.5,125.0,121.0,120.5,114.4,114.3$, 111.7, 105.4, 63.9, 60.2, 55.3; MS (ESI): $m / z 308.1\left[\mathrm{M}+\mathrm{H}^{+}\right]$.

\section{5,6-Dihydro-6-hydroxymethyl-5-(2-methylphenyl)pyrido[2,3-e]pyrrolo[1,2-a]pyr}

azine 3c: $26 \%$. mp: $48-49{ }^{\circ} \mathrm{C} .{ }^{1} \mathrm{H}$ NMR $\delta 7.83(\mathrm{~d}, 1 \mathrm{H}, J=4.5), 7.50(\mathrm{~d}, 1 \mathrm{H}, J=7.2)$, 7.31-7.24 (m, 5H), $6.66(\mathrm{t}, 1 \mathrm{H}, J=6.0), 6.44(\mathrm{~s}, 1 \mathrm{H}), 6.13(\mathrm{~s}, 1 \mathrm{H}), 4.78(\mathrm{br}, 1 \mathrm{H})$, 3.84-3.66 (m, 2H), $2.26(\mathrm{~s}, 1 \mathrm{H}), 2.08(\mathrm{~s}, 3 \mathrm{H}) ; \mathrm{MS}(\mathrm{ESI}): \mathrm{m} / \mathrm{z} 292.3\left[\mathrm{M}+\mathrm{H}^{+}\right]$. 
5,6-Dihydro-6-hydroxymethyl-5-(3-methylphenyl)pyrido[2,3-e]pyrrolo[1,2-a]pyr azine 3d: $81 \%$. mp: $155-157{ }^{\circ} \mathrm{C} .{ }^{1} \mathrm{H}$ NMR $\delta 7.95(\mathrm{~d}, 1 \mathrm{H}, J=5.1), 7.57(\mathrm{~d}, 1 \mathrm{H}, J=$ 7.5), 7.29-7.16 (m, 4H), $6.98(\mathrm{~d}, 1 \mathrm{H}, J=7.2), 6.84-6.79(\mathrm{~m}, 1 \mathrm{H}), 6.41(\mathrm{~s}, 1 \mathrm{H}), 6.09$ (s, $1 \mathrm{H}), 5.01(\mathrm{t}, 1 \mathrm{H}, J=6.0), 3.83-3.58(\mathrm{~m}, 2 \mathrm{H}), 2.35(\mathrm{~s}, 3 \mathrm{H}), 2.06(\mathrm{t}, 1 \mathrm{H}, J=6.6) ;{ }^{13} \mathrm{C}$ NMR $\delta 146.2,144.5,143.5,138.9,128.9,125.6,125.3,124.9,122.1,121.5,121.1$, 115.4, 114.5, 111.8, 105.7, 64.2, 59.8, 21.5; MS (ESI): $\mathrm{m} / z, 292.2\left[\mathrm{M}+\mathrm{H}^{+}\right]$.

5,6-Dihydro-6-hydroxymethyl-5-(4-methylphenyl)pyrido[2,3-e]pyrrolo[1,2-a]pyr azine 3e: $80 \%$. mp: $121-122{ }^{\circ} \mathrm{C} .{ }^{1} \mathrm{H}$ NMR $\delta 7.91(\mathrm{~d}, 1 \mathrm{H}, J=4.5), 7.55(\mathrm{~d}, 1 \mathrm{H}, J=$ 6.6), 7.27-7.17 (m, 5H), 6.79-6.75 (m, 1H), $6.41(\mathrm{t}, 1 \mathrm{H}, J=3.0), 6.09(\mathrm{~d}, 1 \mathrm{H}, J=2.1)$, $5.06(\mathrm{t}, 1 \mathrm{H}, J=6.0), 3.79-3.60(\mathrm{~m}, 2 \mathrm{H}), 2.35(\mathrm{~s}, 3 \mathrm{H}), 2.11(\mathrm{br}, 1 \mathrm{H}) ;{ }^{13} \mathrm{C} \mathrm{NMR} \delta 146.4$, $143.2,141.7,134.4,129.6,125.3,124.7,121.7,120.8,114.9,114.2,111.7,105.5$, 64.0, 59.8, 20.8; MS (ESI): $m / z, 292.2\left[\mathrm{M}+\mathrm{H}^{+}\right]$.

5,6-Dihydro-6-hydroxymethyl-5-phenylpyrido[2,3-e]pyrrolo[1,2-a]pyrazine $3 f$ : 90\%. mp: $65-66{ }^{\circ} \mathrm{C} .{ }^{1} \mathrm{H}$ NMR $\delta 7.95(\mathrm{~d}, 1 \mathrm{H}, J=5.1), 7.58(\mathrm{~d}, 1 \mathrm{H}, J=6.6), 7.36(\mathrm{~d}$, $4 \mathrm{H}, J=3.6), 7.20-7.14(\mathrm{~m}, 2 \mathrm{H}), 6.85-6.81(\mathrm{~m}, 1 \mathrm{H}), 6.41(\mathrm{t}, 1 \mathrm{H}, J=3.0), 6.10(\mathrm{~d}, 1 \mathrm{H}$, $J=2.4), 5.13(\mathrm{t}, 1 \mathrm{H}, J=6.0), 3.79-3.61(\mathrm{~m}, 2 \mathrm{H}), 2.07(\mathrm{br}, 1 \mathrm{H}) ;{ }^{13} \mathrm{C} \mathrm{NMR} \delta 146.0$ $144.6,143.4,129.0,125.4,124.4,124.0,122.4,121.3,115.7,114.5,111.8,105.7$, 64.2, 59.8; MS (ESI): $m / z, 278.2\left[\mathrm{M}+\mathrm{H}^{+}\right]$.

5,6-Dihydro-6-hydroxymethyl-5-(4-bromophenyl)pyrido[2,3-e]pyrrolo[1,2-a]pyr azine 3g: $88 \%$. mp: $173-174{ }^{\circ} \mathrm{C} .{ }^{1} \mathrm{H}$ NMR $\delta 7.95(\mathrm{dd}, 1 \mathrm{H}, J=5.4,1.5), 7.60(\mathrm{dd}, 1 \mathrm{H}$, $J=7.2,1.5), 7.46-7.41(\mathrm{~m}, 2 \mathrm{H}), 7.28-7.19(\mathrm{~m}, 3 \mathrm{H}), 6.89-6.85(\mathrm{~m}, 1 \mathrm{H}), 6.41(\mathrm{t}, 1 \mathrm{H}, J$ $=3.0), 6.10(\mathrm{dd}, 1 \mathrm{H}, J=3.6,1.5), 5.09(\mathrm{t}, 1 \mathrm{H}, J=6.0), 3.76-3.58(\mathrm{~m}, 2 \mathrm{H}), 2.26(\mathrm{br}$, 
$1 \mathrm{H}) ;{ }^{13} \mathrm{C}$ NMR $\delta 145.5,143.7,143.4,131.9,125.4,125.1,122.6,121.5,117.0,116.3$, 114.6, 112.0, 105.9, 64.2, 59.7; MS (ESI): $m / z 356.0\left[\mathrm{M}+\mathrm{H}^{+}\right]$.

5,6-Dihydro-6-hydroxymethyl-5-(3-chlorophenyl)pyrido[2,3-e]pyrrolo[1,2-a]pyra zine 3i: $95 \%$. mp: $68-69{ }^{\circ} \mathrm{C} .{ }^{1} \mathrm{H}$ NMR $\delta 7.99(\mathrm{dd}, 1 \mathrm{H}, J=4.8,1.5), 7.62(\mathrm{dd}, 1 \mathrm{H}, J=$ 7.8, 1.5), 7.38-7.36 (m, 1H), 7.28-7.26 (m, 2H), 7.21-7.19 (m, 1H), 7.18-7.08 (m, 1H), 6.93-6.89 (m, 1H), $6.41(\mathrm{t}, 1 \mathrm{H}, J=3.3), 6.11(\mathrm{dd}, 1 \mathrm{H}, J=3.0,1.2), 5.13(\mathrm{t}, 1 \mathrm{H}, J=$ 6.0), 3.78-3.59 (m, 2H), $2.29(\mathrm{br}, 1 \mathrm{H}) ;{ }^{13} \mathrm{C}$ NMR (DMSO-d6) $\delta 146.0,145.2,142.8$, $132.8,130.0,126.0,123.7,123.2,122.4,122.2,121.9,116.5,115.2,111.4,105.5$, 63.8, 59.1; MS (ESI): $m / z 312.1\left[\mathrm{M}+\mathrm{H}^{+}\right]$.

5,6-Dihydro-6-hydroxymethyl-5-(4-chlorophenyl)pyrido[2,3-e]pyrrolo[1,2-a]pyra zine 3j: $97 \%$. mp: $164-165{ }^{\circ} \mathrm{C} .{ }^{1} \mathrm{H}$ NMR $\delta 7.96(\mathrm{dd}, 1 \mathrm{H}, J=5.1,1.2), 7.60(\mathrm{dd}, 1 \mathrm{H}, J$ $=8.1,1.5), 7.32-7.29(\mathrm{~m}, 4 \mathrm{H}), 7.20(\mathrm{~s}, 1 \mathrm{H}), 6.89-6.85(\mathrm{~m}, 1 \mathrm{H}), 6.42(\mathrm{t}, 1 \mathrm{H}, J=3.0)$,

6.11-6.10 (m, 1H), $5.09(\mathrm{t}, 1 \mathrm{H}, J=6.0), 3.78-3.59(\mathrm{~m}, 2 \mathrm{H}) ;{ }^{13} \mathrm{C} \mathrm{NMR} \delta 145.6,143.5$, $143.1,129.4,129.0,125.1,122.5,121.5,116.2,114.6,112.0,105.9,64.2,59.8 ; \mathrm{MS}$ (ESI): $m / z 312.2\left[\mathrm{M}+\mathrm{H}^{+}\right]$. Anal. Calcd for $\mathrm{C}_{17} \mathrm{H}_{14} \mathrm{ClN}_{3} \mathrm{O}: \mathrm{C}, 65.49 ; \mathrm{H}, 4.53 ; \mathrm{N}, 13.48$. Found: C, 65.58; H, 4.73; N, 13.32 .

\section{5,6-Dihydro-6-hydroxymethyl-5-(4-acetylphenyl)pyrido[2,3-e $]$ pyrrolo[1,2-a]pyra}

zine 3k: $80 \%$. mp: $79-80{ }^{\circ} \mathrm{C} .{ }^{1} \mathrm{H}$ NMR $\delta 8.03(\mathrm{~d}, 1 \mathrm{H}, J=5.1), 7.91(\mathrm{~d}, 2 \mathrm{H}, J=8.7)$, $7.65(\mathrm{~d}, 1 \mathrm{H}, J=8.1), 7.40(\mathrm{~d}, 2 \mathrm{H}, J=8.1), 7.18(\mathrm{~s}, 1 \mathrm{H}), 7.01-6.97(\mathrm{~m}, 1 \mathrm{H}), 6.39(\mathrm{t}$, $1 \mathrm{H}, J=3.0), 6.10(\mathrm{~s}, 1 \mathrm{H}), 5.25(\mathrm{t}, 1 \mathrm{H}, J=6.6), 3.73-3.59(\mathrm{~m}, 2 \mathrm{H}), 2.55(\mathrm{~s}, 3 \mathrm{H}), 2.44$ (br, 1H); ${ }^{13} \mathrm{C}$ NMR $\delta 196.8,149.2,144.5,143.6,131.7,129.5,125.3,123.7,122.3$, 120.7, 118.0, 114.8, 112.0, 106.4, 64.2, 59.0, 26.4; MS (ESI): $m / z 320.1\left[\mathrm{M}+\mathrm{H}^{+}\right]$. 
Anal. Calcd for $\mathrm{C}_{19} \mathrm{H}_{17} \mathrm{~N}_{3} \mathrm{O}_{2}$ : C, 71.46; H, 5.37; N, 13.16. Found: C, 71.40; H, 5.60; N, 13.10.

\section{5,6-Dihydro-6-hydroxymethyl-5-(4-cyanophenyl)pyrido[2,3-e]pyrrolo[1,2-a]pyra}

zine 31: 58\%. mp: 209-210 ${ }^{\circ} \mathrm{C} .{ }^{1} \mathrm{H}$ NMR $\delta 8.06(\mathrm{~d}, 1 \mathrm{H}, J=4.5), 7.69(\mathrm{~d}, 1 \mathrm{H}, J=8.1)$, $7.57(\mathrm{~d}, 2 \mathrm{H}, J=9.0), 7.45(\mathrm{~d}, 2 \mathrm{H}, J=8.7), 7.20(\mathrm{~s}, 1 \mathrm{H}), 7.07-7.03(\mathrm{~m}, 1 \mathrm{H}), 6.41(\mathrm{t}$, $1 \mathrm{H}, J=3.0), 6.13(\mathrm{~d}, 1 \mathrm{H}, J=2.7), 5.25(\mathrm{t}, 1 \mathrm{H}, J=7.2), 3.74-3.62(\mathrm{~m}, 2 \mathrm{H}), 2.34(\mathrm{t}$, $1 \mathrm{H}, J=6.6) ;{ }^{13} \mathrm{C}$ NMR (DMSO- $d 6$ ) $\delta 153.9,149.0,148.2,137.8,131.1,128.6,128.0$, 126.7, 124.6, 123.5, 120.7, 116.6, 111.1, 108.6, 68.9, 63.3; MS (ESI): $m / z 303.1$ $\left[\mathrm{M}+\mathrm{H}^{+}\right]$. Anal. Calcd for $\mathrm{C}_{18} \mathrm{H}_{14} \mathrm{~N}_{4} \mathrm{O}: \mathrm{C}, 71.51 ; \mathrm{H}, 4.67 ; \mathrm{N}, 18.53$. Found: $\mathrm{C}, 71.40 ; \mathrm{H}$, $4.78 ; \mathrm{N}, 18.39$.

\section{5,6-Dihydro-6-hydroxymethyl-5-(4-nitrophenyl)pyrido[2,3-e]pyrrolo[1,2-a]pyraz}

ine 3m: $38 \%$. mp: $187-188{ }^{\circ} \mathrm{C} .{ }^{1} \mathrm{H}$ NMR $\delta 8.17(\mathrm{~d}, 2 \mathrm{H}, J=8.7), 8.10(\mathrm{~d}, 1 \mathrm{H}, J=5.1)$, $7.71(\mathrm{~d}, 1 \mathrm{H}, J=8.1), 7.47(\mathrm{~d}, 2 \mathrm{H}, J=9.6), 7.20(\mathrm{~s}, 1 \mathrm{H}), 7.12-7.08(\mathrm{~m}, 1 \mathrm{H}), 6.42(\mathrm{t}$, $1 \mathrm{H}, J=3.0), 6.15(\mathrm{~s}, 1 \mathrm{H}), 5.32(\mathrm{t}, 1 \mathrm{H}, J=6.0), 3.69(\mathrm{~s}, 2 \mathrm{H}), 2.38(\mathrm{br}, 1 \mathrm{H}) ;{ }^{13} \mathrm{C} \mathrm{NMR}$ (DMSO-d6) $\delta 150.8,143.4,143.1,140.8,125.8,124.4,123.8,123.1,120.4,119.1$, 115.7, 111.4, 106.1, 63.7, 58.1; MS (ESI): $m / z 323.1\left[\mathrm{M}+\mathrm{H}^{+}\right]$. Anal. Calcd for $\mathrm{C}_{17} \mathrm{H}_{14} \mathrm{~N}_{4} \mathrm{O}_{3}: \mathrm{C}, 63.35 ; \mathrm{H}, 4.38 ; \mathrm{N}, 17.38$. Found: $\mathrm{C}, 63.50 ; \mathrm{H}, 4.45 ; \mathrm{N}, 17.26$.

Pyrido[2,3-b]pyrrolo[1,2-d][1,4]oxazepine 7: 17\%. mp: 96-97 ${ }^{\circ} \mathrm{C} .{ }^{1} \mathrm{H}$ NMR $\delta 8.15$ (d, 1H, $J=4.2), 7.65(\mathrm{~d}, 1 \mathrm{H}, J=7.5), 7.20-7.16(\mathrm{~m}, 1 \mathrm{H}), 6.99(\mathrm{~s}, 1 \mathrm{H}), 6.52(\mathrm{~d}, 1 \mathrm{H}, J=$ 5.7), $6.36(\mathrm{~d}, 1 \mathrm{H}, J=3.0), 6.22(\mathrm{~s}, 1 \mathrm{H}), 6.01(\mathrm{~d}, 1 \mathrm{H}, J=5.7)$; MS (ESI): $m / z 185.1$ $\left[\mathrm{M}+\mathrm{H}^{+}\right]$. 
4-Chlorophenylamino-6,7-dihydropyrido[2,3-b]pyrrolo[1,2-d][1,4]oxazepine $2 \mathrm{j}$ :

48\%. mp: $143-145{ }^{\circ} \mathrm{C} .{ }^{1} \mathrm{H}$ NMR $\delta 8.24(\mathrm{~d}, 1 \mathrm{H}, J=5.1), 7.76(\mathrm{~d}, 1 \mathrm{H}, J=7.8)$, 7.26-7.22 (m, 1H), $7.08(\mathrm{~d}, 2 \mathrm{H}, J=8.7), 6.98(\mathrm{~s}, 1 \mathrm{H}), 6.50(\mathrm{~d}, 2 \mathrm{H}, J=8.7), 6.24(\mathrm{t}$, $1 \mathrm{H}, J=3.0), 6.22(\mathrm{~s}, 1 \mathrm{H}), 4.86-4.80(\mathrm{~m}, 2 \mathrm{H}), 4.51-4.44(\mathrm{~m}, 1 \mathrm{H}), 3.88(\mathrm{~d}, 1 \mathrm{H}, J=7.5)$; ${ }^{13} \mathrm{C}$ NMR $\delta 155.4,145.5,144.6,131.7,131.4,129.0,128.1,123.1,121.1,120.7$, 114.8, 110.2, 108.5, 77.2, 50.4; MS (ESI): $m / z 312.1\left[\mathrm{M}+\mathrm{H}^{+}\right]$.

$\mathrm{TiCl}_{4}$-promoted reaction for the synthesis of pyrido[2,3-e]pyrrolo[1,2-a] pyrazine 3: To a stirred solution of 2-(3-(1H-pyrrol-1-yl)pyridin-2-yloxy)acetaldehyde 1 (101 $\mathrm{mg}, 0.5 \mathrm{mmol})$ in DCM $(20 \mathrm{~mL})$ was added the aliphatic amine $(0.55 \mathrm{mmol})$. After 10 min, $\mathrm{TiCl}_{4}(132 \mu \mathrm{L}, 1.2 \mathrm{mmol})$ was added. The resulting solution was stirred for the corresponding time at ambient temperature, quenched with saturated aqueous $\mathrm{NaHCO}_{3}$, extracted with $\mathrm{DCM}(30 \mathrm{~mL})$, dried over $\mathrm{MgSO}_{4}$, and concentrated in vacuo. Purification by flash chromatography (Petroleum ether/EtOAc $=3: 1, \mathrm{v} / \mathrm{v}$ ) afforded the desired products.

5-(n-Butyl)-5,6-dihydro-6-hydroxymethylpyrido[2,3-e]pyrrolo[1,2-a]pyrazine 3n: 66\%. ${ }^{1} \mathrm{H}$ NMR $\delta 7.88(\mathrm{~d}, 1 \mathrm{H}, J=4.2), 7.35(\mathrm{~d}, 1 \mathrm{H}, J=7.5), 7.10(\mathrm{~s}, 1 \mathrm{H}), 6.59-6.55$ $(\mathrm{m}, 1 \mathrm{H}), 6.33(\mathrm{t}, 1 \mathrm{H}, J=3.0), 6.05(\mathrm{~d}, 1 \mathrm{H}, J=3.0), 4.69-4.65(\mathrm{~m}, 1 \mathrm{H}), 4.27-4.20(\mathrm{~m}$, 1H), 3.71-3.49 (m, 2H), 3.15-3.06 (m, 1H), 1.64-1.55 (m, 2H), 1.39-1.29 (m, 2H), $0.88(\mathrm{t}, 3 \mathrm{H}, J=7.2) ;{ }^{13} \mathrm{C}$ NMR $\delta 147.2,143.0,125.0,120.3,119.3,114.2,112.1$, 111.4, 105.2, 63.9, 57.2, 46.7 29.9, 20.0, 13.9; MS (ESI): $m / z .258 .1\left[\mathrm{M}+\mathrm{H}^{+}\right]$.

\section{5,6-Dihydro-6-hydroxymethyl-5-(iso-propyl)pyrido[2,3-e]pyrrolo[1,2-a]pyrazine}

3o: $61 \%$. mp: $79-80{ }^{\circ} \mathrm{C} .{ }^{1} \mathrm{H}$ NMR $\delta 7.99(\mathrm{dd}, 1 \mathrm{H}, J=5.1,1.5), 7.46(\mathrm{dd}, 1 \mathrm{H}, J=7.8$, 
1.5), 7.15-7.14 (m, 1H), 6.69-6.65 (m, 1H), $6.38(\mathrm{t}, 1 \mathrm{H}, J=3.0), 6.10(\mathrm{dd}, 1 \mathrm{H}, J=3.9$, 1.5), 4.85-4.73 (m, 2H), 3.63-3.57 (m, 1H), 3.40-3.33 (m, 1H), $1.41(\mathrm{~d}, 3 \mathrm{H}, J=6.6)$, $1.24(\mathrm{~d}, 3 \mathrm{H}, J=6.6) ;{ }^{13} \mathrm{C}$ NMR $\delta 147.7,143.3,125.4,121.0,120.0,114.7,112.8$, 111.6, 105.1, 64.9, 51.3, 48.4, 21.9, 20.6; MS (ESI): $m / z 244.2\left[\mathrm{M}+\mathrm{H}^{+}\right]$.

\section{5-Cyclohexyl-5,6-dihydro-6-hydroxymethylpyrido[2,3-e]pyrrolo[1,2-a]pyrazine}

3p: 38\%. ${ }^{1} \mathrm{H}$ NMR $\delta 7.99(\mathrm{dd}, 1 \mathrm{H}, J=5.1,1.5), 7.44(\mathrm{~d}, 1 \mathrm{H}, J=7.5), 7.12(\mathrm{~s}, 1 \mathrm{H})$, 6.68-6.64 (m, 1H), $6.37(\mathrm{t}, 1 \mathrm{H}, J=3.0), 6.07(\mathrm{~d}, 1 \mathrm{H}, J=1.2), 4.75-4.70(\mathrm{~m}, 1 \mathrm{H})$, 4.49-4.41 (m, 1H), 3.53 (br, 1H), 3.32 (t, 1H, $J=9.0), 2.14-2.10(\mathrm{~m}, 1 \mathrm{H}), 1.90-1.87$

$(\mathrm{m}, 1 \mathrm{H}), 1.79-1.48(\mathrm{~m}, 6 \mathrm{H}), 1.43-1.12(\mathrm{~m}, 2 \mathrm{H}) ;{ }^{13} \mathrm{C}$ NMR $\delta 147.7,143.3,125.7$, $121.3,120.1,114.6,113.0,111.6,104.9,65.0,56.9,51.8,32.4,31.2,26.1,26.0,25.7$; MS (ESI): $m / z$ 284.1 [M+H'].

5-Benzyl-5,6-dihydro-6-hydroxymethylpyrido[2,3-e]pyrrolo[1,2-a]pyrazine $3 q$ : 31\%. ${ }^{1} \mathrm{H}$ NMR $\delta 7.94(\mathrm{~d}, 1 \mathrm{H}, J=5.1), 7.45(\mathrm{~d}, 1 \mathrm{H}, J=8.1), 7.32-7.25(\mathrm{~m}, 5 \mathrm{H}), 7.17$ $(\mathrm{s}, 1 \mathrm{H}), 6.70-6.66(\mathrm{~m}, 1 \mathrm{H}), 6.37(\mathrm{t}, 1 \mathrm{H}, J=3.0), 6.01(\mathrm{~d}, 1 \mathrm{H}, J=1.5), 5.60(\mathrm{~d}, 1 \mathrm{H}, J=$ 15.3), $4.64(\mathrm{t}, 1 \mathrm{H}, J=5.1), 4.46(\mathrm{~d}, 1 \mathrm{H}, J=15.3), 3.68-3.60(\mathrm{~m}, 2 \mathrm{H}) ;{ }^{13} \mathrm{C} \mathrm{NMR} \delta$ $147.5,143.0,137.9,128.6,127.7,127.2,125.0,120.3,119.7,114.3,113.0,111.5$, 105.4, 63.7, 56.0, 49.4; MS (ESI): $m / z 292.1\left[\mathrm{M}+\mathrm{H}^{+}\right]$. 
Figure Legends:

Fig. S-1: LC-MS-ELSD of 2-chloro-3-(1H-pyrrol-1-yl)pyridine 5.

Fig. S-2: ${ }^{1} \mathrm{H}$ Spectra of 2-chloro-3-(1H-pyrrol-1-yl)pyridine 5.

Fig. S-3: LC-MS-ELSD of 2-hydroxyethoxy-3-pyrrol-1-yl-pyridine 6.

Fig. S-4: ${ }^{1} \mathrm{H}$ Spectra of 2-hydroxyethoxy-3-pyrrol-1-yl-pyridine 6.

Fig. S-5: ${ }^{13} \mathrm{C}$ Spectra of 2-hydroxyethoxy-3-pyrrol-1-yl-pyridine 6.

Fig. S-6: ${ }^{1} \mathrm{H}$ Spectra of 2-(3-(1H-pyrrol-1-yl)pyridin-2-yloxy)acetaldehyde 1.

Fig. S-7: LC-MS-ELSD of 5,6-dihydro-6-hydroxymethyl-5-(2-methoxylphenyl) pyrido[2,3-e]pyrrolo[1,2-a]pyrazine 3a.

Fig. S-8: ${ }^{1} \mathrm{H}$ Spectra of 5,6-dihydro-6-hydroxymethyl-5-(2-methoxylphenyl) pyrido[2,3-e $]$ pyrrolo[1,2-a]pyrazine 3a.

Fig. S-9: ${ }^{13} \mathrm{C}$ Spectra of 5,6-dihydro-6-hydroxymethyl-5-(2-methoxylphenyl) pyrido[2,3-e]pyrrolo[1,2-a]pyrazine 3a.

Fig. S-10: LC-MS-ELSD of 5,6-dihydro-6-hydroxymethyl-5-(4-methoxylphenyl) pyrido[2,3-e $]$ pyrrolo[1,2-a]pyrazine $3 \mathrm{~b}$.

Fig. S-11: ${ }^{1} \mathrm{H}$ Spectra of 5,6-dihydro-6-hydroxymethyl-5-(4-methoxylphenyl) pyrido[2,3-e]pyrrolo[1,2-a]pyrazine $3 \mathrm{~b}$.

Fig. S-12: ${ }^{13} \mathrm{C}$ Spectra of 5,6-dihydro-6-hydroxymethyl-5-(4-methoxylphenyl) pyrido[2,3-e $]$ pyrrolo[1,2-a]pyrazine $3 \mathrm{~b}$.

Fig. S-13: LC-MS-ELSD of 5,6-dihydro-6-hydroxymethyl-5-(2-methylphenyl) pyrido[2,3-e $]$ pyrrolo[1,2-a]pyrazine $3 c$.

Fig. S-14: ${ }^{1} \mathrm{H}$ Spectra of 5,6-Dihydro-6-hydroxymethyl-5-(2-methylphenyl) pyrido[2,3-e]pyrrolo[1,2-a]pyrazine $3 \mathrm{c}$.

Fig. S-15: LC-MS-ELSD of 5,6-dihydro-6-hydroxymethyl-5-(3-methylphenyl) pyrido $[2,3-e]$ pyrrolo $[1,2-a]$ pyrazine $3 \mathrm{~d}$.

Fig. S-16: ${ }^{1} \mathrm{H}$ Spectra of 5,6-dihydro-6-hydroxymethyl-5-(3-methylphenyl) pyrido[2,3-e]pyrrolo[1,2-a]pyrazine 3d.

Fig. S-17: ${ }^{13} \mathrm{C}$ Spectra of 5,6-dihydro-6-hydroxymethyl-5-(3-methylphenyl) pyrido[2,3-e]pyrrolo[1,2-a]pyrazine 3d. 
Fig. S-18: LC-MS-ELSD of 5,6-dihydro-6-hydroxymethyl-5-(4-methylphenyl) pyrido[2,3-e]pyrrolo[1,2-a]pyrazine $3 \mathrm{e}$.

Fig. S-19: ${ }^{1} \mathrm{H}$ Spectra of 5,6-dihydro-6-hydroxymethyl-5-(4-methylphenyl) pyrido[2,3-e $]$ pyrrolo[1,2-a]pyrazine $3 \mathrm{e}$.

Fig. S-20: ${ }^{13} \mathrm{C}$ Spectra of 5,6-dihydro-6-hydroxymethyl-5-(4-methylphenyl) pyrido[2,3-e $]$ pyrrolo[1,2-a]pyrazine $3 \mathrm{e}$.

Fig. S-21: LC-MS-ELSD of 5,6-dihydro-6-hydroxymethyl-5-phenylpyrido[2,3-e] pyrrolo[1,2-a]pyrazine $3 f$.

Fig. S-22: ${ }^{1} \mathrm{H}$ Spectra of 5,6-dihydro-6-hydroxymethyl-5-phenylpyrido[2,3-e] pyrrolo[1,2-a]pyrazine 3f.

Fig. S-23: ${ }^{13} \mathrm{C}$ Spectra of 5,6-dihydro-6-hydroxymethyl-5-phenylpyrido[2,3-e ] pyrrolo[1,2-a]pyrazine 3f.

Fig. S-24: LC-MS-ELSD of 5,6-dihydro-6-hydroxymethyl-5-(4-bromophenyl) pyrido[2,3-e]pyrrolo[1,2-a]pyrazine 3g.

Fig. S-25: ${ }^{1} \mathrm{H}$ Spectra of 5,6-dihydro-6-hydroxymethyl-5-(4-bromophenyl) pyrido[2,3-e]pyrrolo[1,2-a]pyrazine 3g.

Fig. S-26: ${ }^{13} \mathrm{C}$ Spectra of 5,6-dihydro-6-hydroxymethyl-5-(4-bromophenyl) pyrido[2,3-e]pyrrolo[1,2-a]pyrazine 3g.

Fig. S-27: LC-MS-ELSD of 5,6-dihydro-6-hydroxymethyl-5-(3-chlorophenyl) pyrido[2,3-e]pyrrolo[1,2-a]pyrazine $3 \mathrm{i}$.

Fig. S-28: ${ }^{1} \mathrm{H}$ Spectra of 5,6-dihydro-6-hydroxymethyl-5-(3-chlorophenyl) pyrido[2,3-e]pyrrolo[1,2-a]pyrazine $3 \mathrm{i}$.

Fig. S-29: ${ }^{13} \mathrm{C}$ Spectra of 5,6-dihydro-6-hydroxymethyl-5-(3-chlorophenyl) pyrido[2,3-e]pyrrolo[1,2-a]pyrazine $3 \mathrm{i}$.

Fig. S-30: LC-MS-ELSD of 5,6-dihydro-6-hydroxymethyl-5-(4-chlorophenyl) pyrido[2,3-e]pyrrolo[1,2-a]pyrazine $3 \mathbf{j}$.

Fig. S-31: ${ }^{1} \mathrm{H}$ Spectra of 5,6-dihydro-6-hydroxymethyl-5-(4-chlorophenyl) pyrido[2,3-e $]$ pyrrolo[1,2-a]pyrazine $3 \mathrm{j}$.

Fig. S-32: ${ }^{13} \mathrm{C}$ Spectra of 5,6-dihydro-6-hydroxymethyl-5-(4-chlorophenyl) pyrido[2,3-e $]$ pyrrolo[1,2-a]pyrazine $3 \mathbf{j}$. 
Fig. S-33: LC-MS-ELSD of 5,6-dihydro-6-hydroxymethyl-5-(4-acetylphenyl) pyrido[2,3-e]pyrrolo[1,2-a]pyrazine $3 \mathrm{k}$.

Fig. S-34: ${ }^{1} \mathrm{H}$ Spectra of 5,6-dihydro-6-hydroxymethyl-5-(4-acetylphenyl) pyrido[2,3-e]pyrrolo[1,2-a]pyrazine $3 \mathrm{k}$.

Fig. S-35: ${ }^{13} \mathrm{C}$ Spectra of 5,6-dihydro-6-hydroxymethyl-5-(4-acetylphenyl) pyrido[2,3-e]pyrrolo[1,2-a]pyrazine $3 \mathrm{k}$.

Fig. S-36: LC-MS-ELSD of 5,6-dihydro-6-hydroxymethyl-5-(4-cyanophenyl) pyrido[2,3-e]pyrrolo[1,2-a]pyrazine 31 .

Fig. S-37: ${ }^{1} \mathrm{H}$ Spectra of 5,6-dihydro-6-hydroxymethyl-5-(4-cyanophenyl) pyrido[2,3-e]pyrrolo[1,2-a]pyrazine 31 .

Fig. S-38: ${ }^{13} \mathrm{C}$ Spectra of 5,6-dihydro-6-hydroxymethyl-5-(4-cyanophenyl) pyrido[2,3-e $]$ pyrrolo[1,2-a]pyrazine 31 .

Fig. S-39: LC-MS-ELSD of 5,6-dihydro-6-hydroxymethyl-5-(4-nitrophenyl) pyrido[2,3-e]pyrrolo[1,2-a]pyrazine $3 \mathrm{~m}$.

Fig. S-40: ${ }^{1} \mathrm{H} \quad$ Spectra of 5,6-dihydro-6-hydroxymethyl-5-(4-nitrophenyl) pyrido[2,3-e]pyrrolo[1,2-a]pyrazine $3 \mathrm{~m}$.

Fig. S-41: ${ }^{13} \mathrm{C}$ Spectra of 5,6-dihydro-6-hydroxymethyl-5-(4-nitrophenyl) pyrido[2,3-e]pyrrolo[1,2-a]pyrazine $3 \mathrm{~m}$.

Fig. S-42: LC-MS-ELSD of pyrido[2,3-b]pyrrolo[1,2-d][1,4]oxazepine 7.

Fig. S-43: ${ }^{1} \mathrm{H}$ Spectra of pyrido[2,3-b]pyrrolo[1,2-d][1,4] oxazepine 7.

Fig. S-44: LC-MS-ELSD of 4-chlorophenylamino-6,7-dihydropyrido[2,3-b] pyrrolo[1,2-d][1,4]oxazepine $2 \mathrm{j}$.

Fig. S-45: ${ }^{1} \mathrm{H}$ Spectra of 4-chlorophenylamino-6,7-dihydropyrido[2,3-b] pyrrolo[1,2-d][1,4]oxazepine $2 \mathrm{j}$.

Fig. S-46: ${ }^{13} \mathrm{C}$ Spectra of 4-chlorophenylamino-6,7-dihydropyrido[2,3-b] pyrrolo[1,2-d][1,4]oxazepine $2 \mathrm{j}$.

Fig. S-47: LC-MS-ELSD of 5-(n-butyl)-5,6-dihydro-6-hydroxymethylpyrido [2,3-e]pyrrolo[1,2-a]pyrazine 3n.

Fig. S-48: ${ }^{1} \mathrm{H}$ Spectra of 5-(n-butyl)-5,6-dihydro-6-hydroxymethylpyrido [2,3-e]pyrrolo[1,2-a]pyrazine 3n. 
Fig. S-49: ${ }^{13} \mathrm{C}$ Spectra of 5-(n-butyl)-5,6-dihydro-6-hydroxymethylpyrido [2,3-e]pyrrolo[1,2-a]pyrazine $3 \mathrm{n}$.

Fig. S-50: LC-MS-ELSD of 5,6-dihydro-6-hydroxymethyl-5-(iso-propyl)pyrido [2,3-e]pyrrolo[1,2-a]pyrazine 30.

Fig. S-51: ${ }^{1} \mathrm{H}$ Spectra of 5,6-dihydro-6-hydroxymethyl-5-(iso-propyl)pyrido [2,3-e]pyrrolo[1,2-a]pyrazine 30 .

Fig. S-52: ${ }^{13} \mathrm{C}$ Spectra of 5,6-dihydro-6-hydroxymethyl-5-(iso-propyl)pyrido [2,3-e]pyrrolo[1,2-a]pyrazine 30 .

Fig. S-53: LC-MS-ELSD of 5-cyclohexyl-5,6-dihydro-6-hydroxymethylpyrido $[2,3-e]$ pyrrolo $[1,2-a]$ pyrazine $3 p$.

Fig. S-54: ${ }^{1} \mathrm{H}$ Spectra of 5-cyclohexyl-5,6-dihydro-6-hydroxymethylpyrido [2,3-e]pyrrolo[1,2-a]pyrazine 3p.

Fig. S-55: ${ }^{13} \mathrm{C}$ Spectra of 5-cyclohexyl-5,6-dihydro-6-hydroxymethylpyrido $[2,3-e]$ pyrrolo $[1,2-a]$ pyrazine $3 p$.

Fig. S-56: LC-MS-ELSD of 5-benzyl-5,6-dihydro-6-hydroxymethylpyrido [2,3-e]pyrrolo[1,2-a]pyrazine 3q.

Fig. S-57: ${ }^{1} \mathrm{H}$ Spectra of 5-benzyl-5,6-dihydro-6-hydroxymethylpyrido [2,3-e]pyrrolo[1,2-a]pyrazine 3q.

Fig. S-58: ${ }^{13} \mathrm{C}$ Spectra of 5-benzyl-5,6-dihydro-6-hydroxylmethypyrido $[2,3-e]$ pyrrolo $[1,2-a]$ pyrazine $3 q$. 


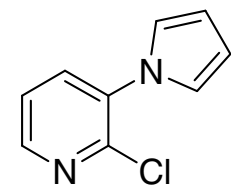

$\mathrm{C}_{9} \mathrm{H}_{7} \mathrm{CIN}_{2}$

Exact Mass: 178.03

5 Mol. Wt.: 178.62

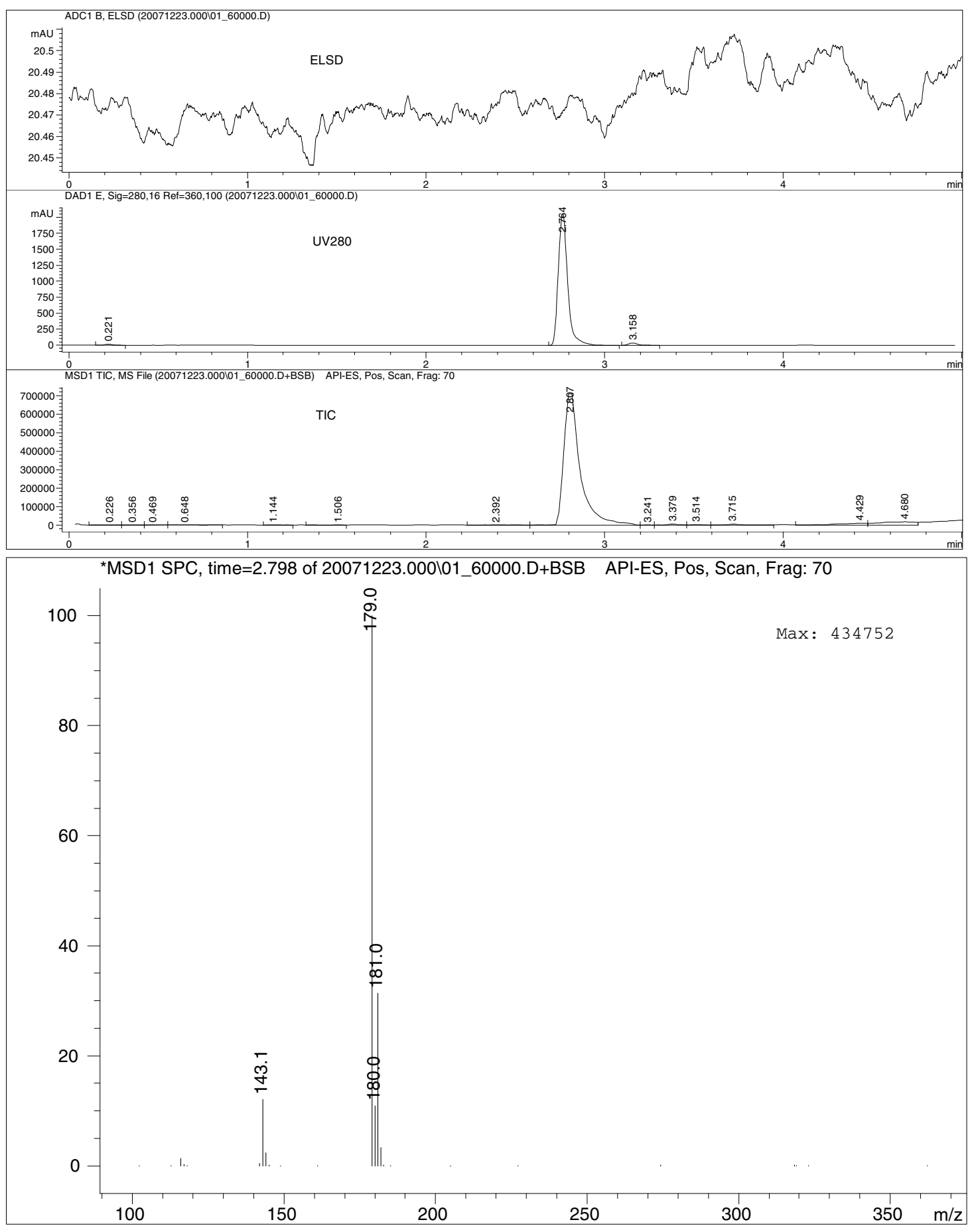

Fig. S-1: LC-MS-ELSD of 2-chloro-3-(1H-pyrrol-1-yl)pyridine 5. 


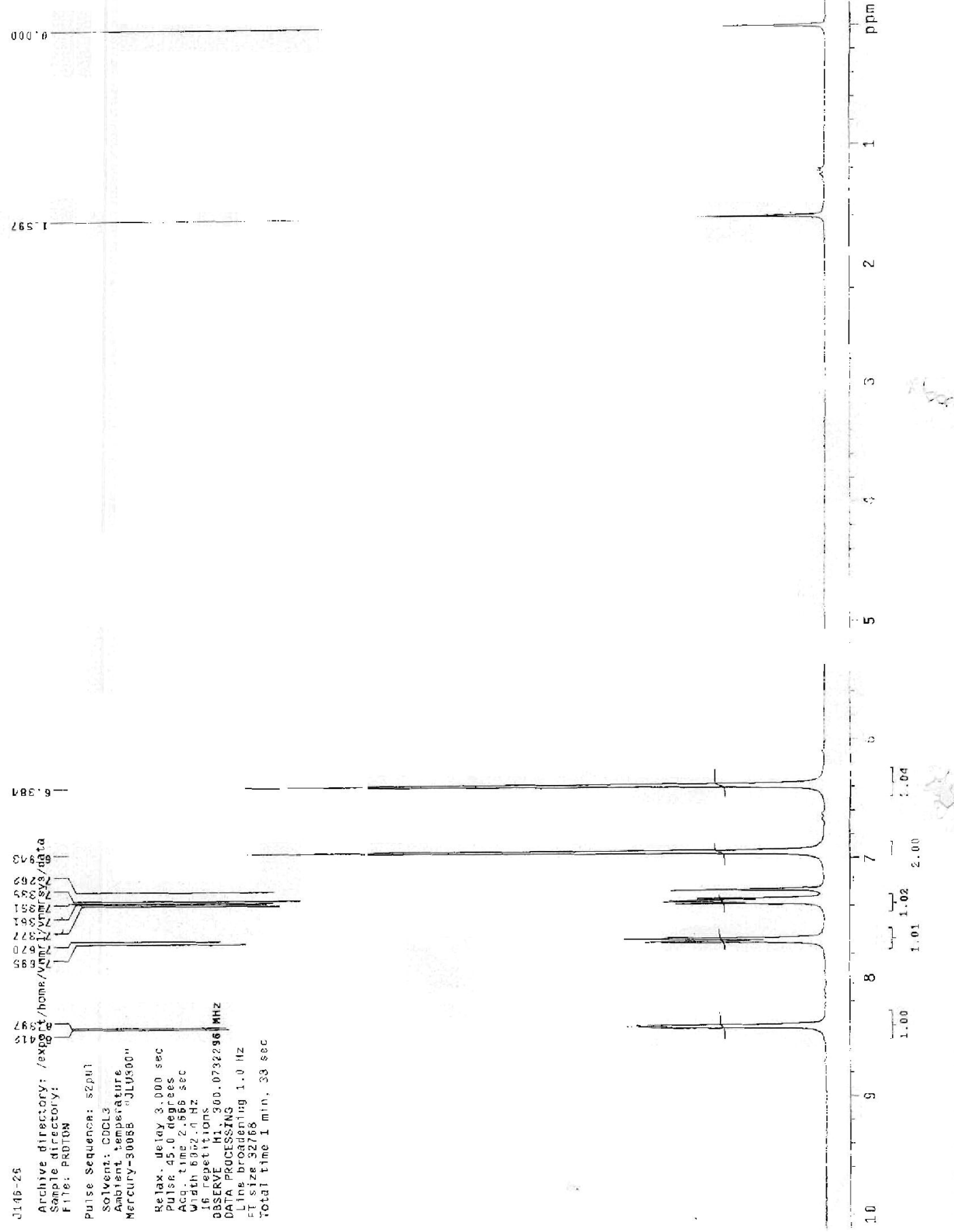

Fig. S-2: ${ }^{1} \mathrm{H}$ Spectra of 2-chloro-3-(1H-pyrrol-1-yl)pyridine 5. 


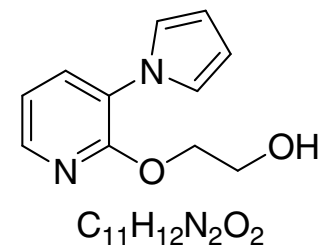

Exact Mass: 204.09

6 Mol. Wt.: 204.23
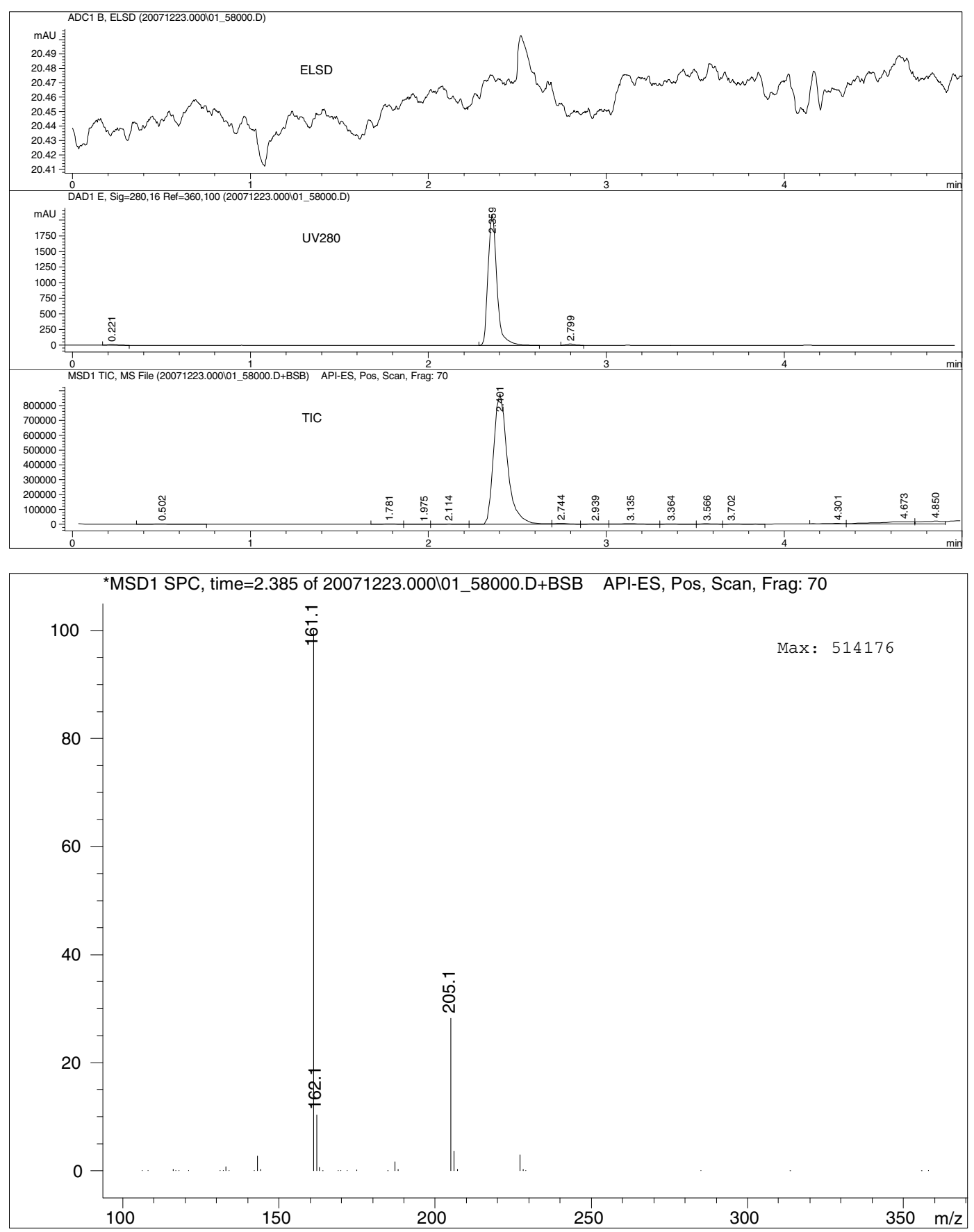

Fig. S-3: LC-MS-ELSD of 2-hydroxyethoxy-3-pyrrol-1-yl-pyridine 6. 


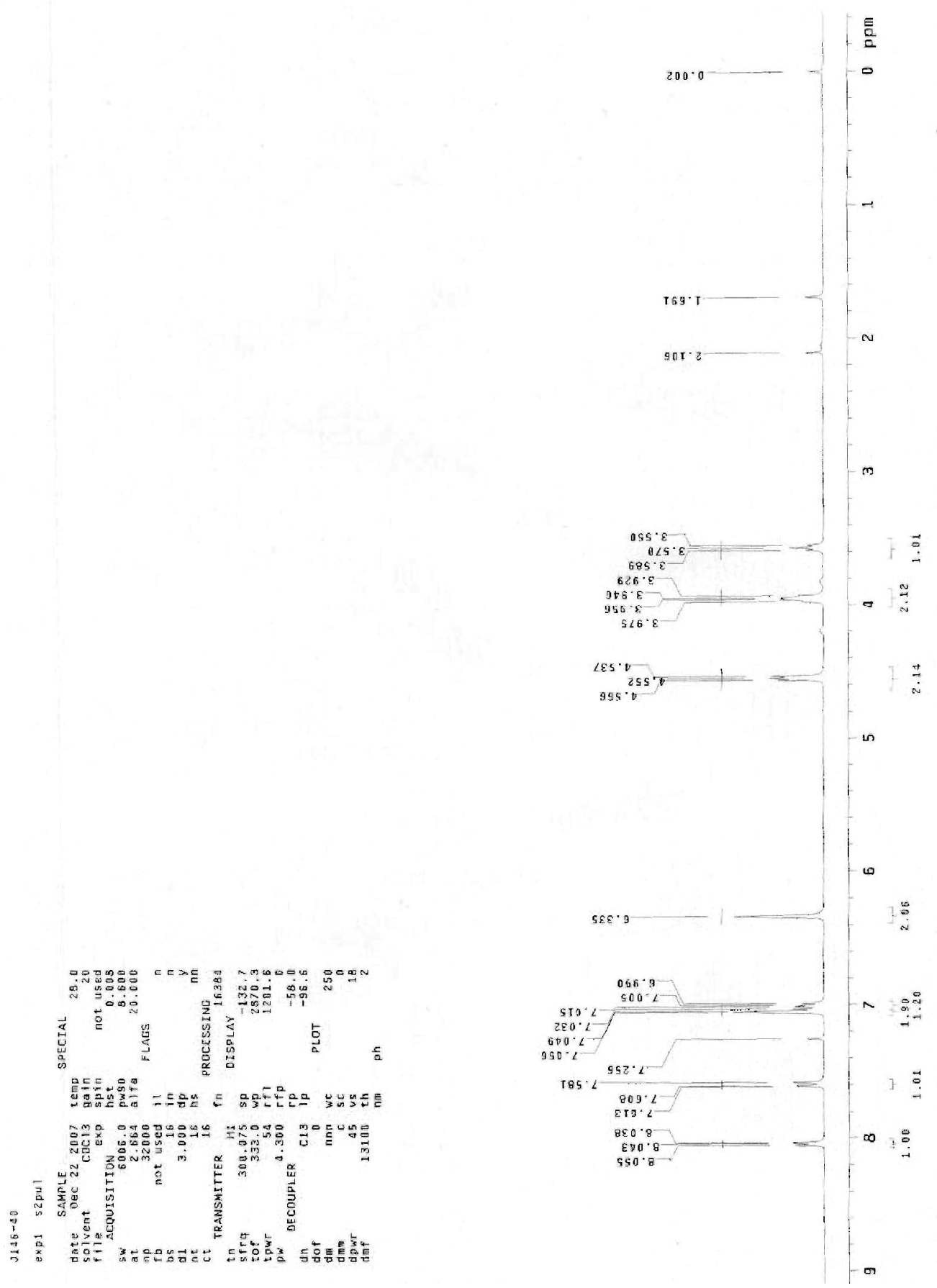

Fig. S-4: ${ }^{1} \mathrm{H}$ Spectra of 2-hydroxyethoxy-3-pyrrol-1-yl-pyridine 6. 


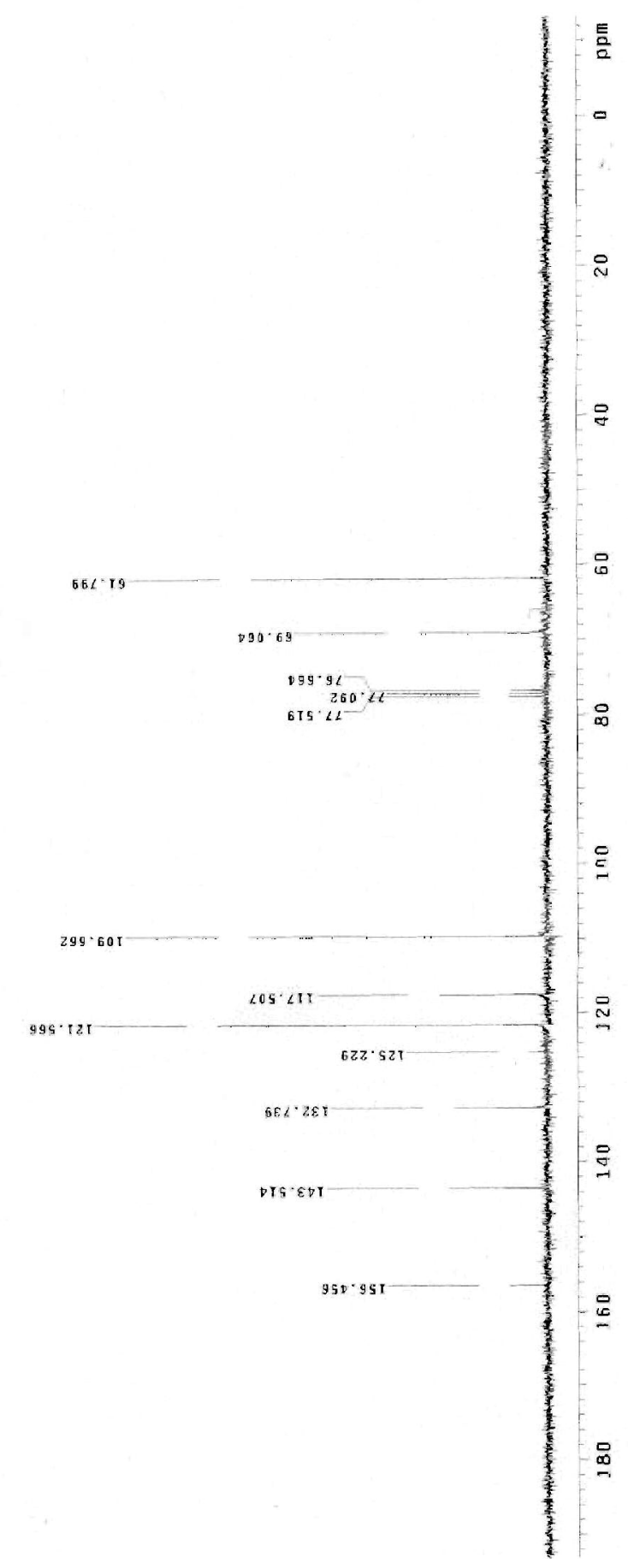

Fig. S-5: ${ }^{13}$ C Spectra of 2-hydroxyethoxy-3-pyrrol-1-yl-pyridine 6. 


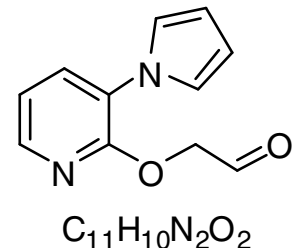

Exact Mass: 202.07

1 Molecular Weight: 202.21

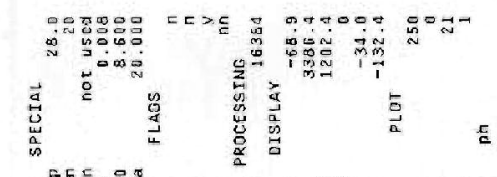

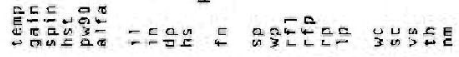
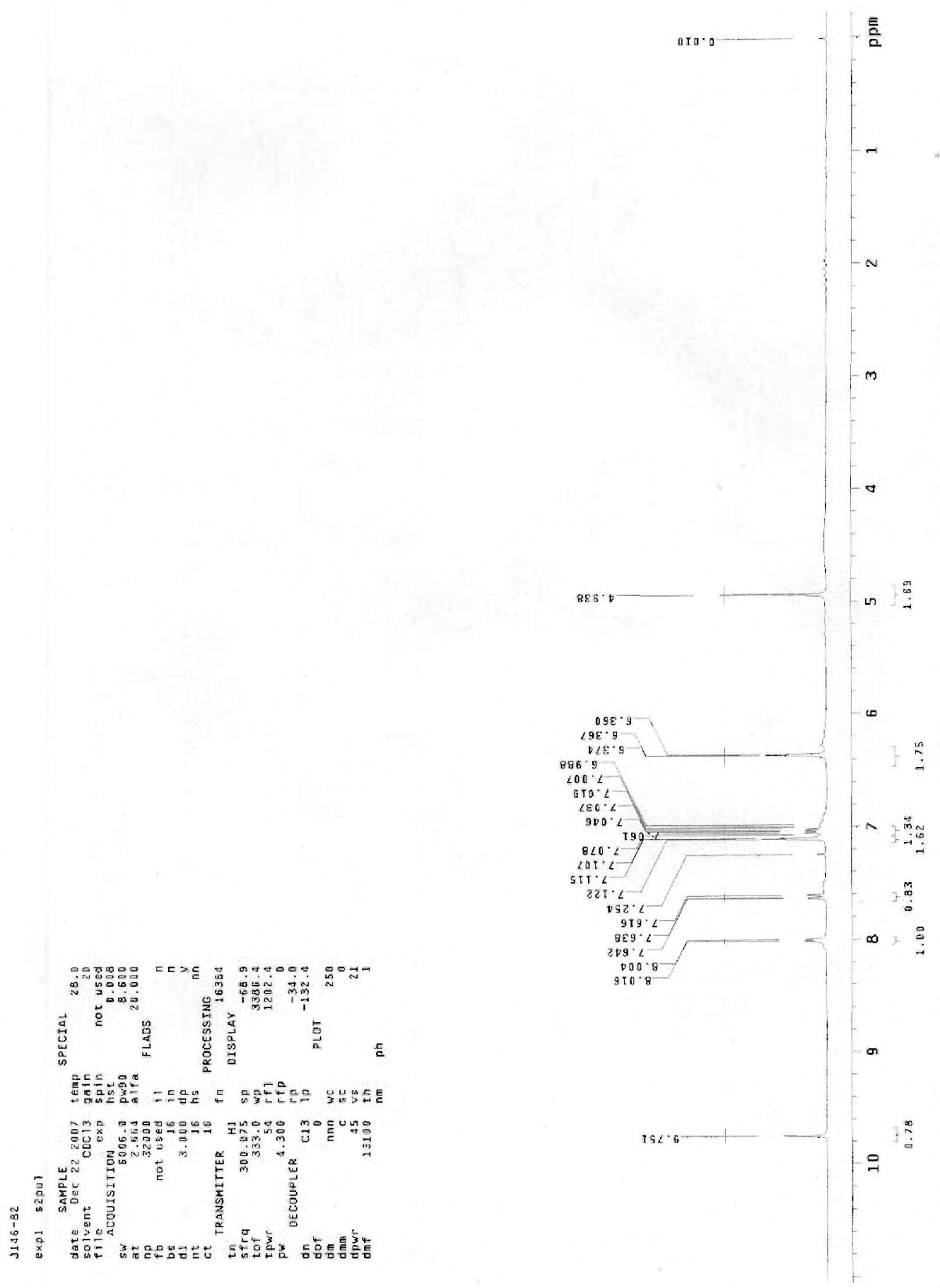

Fig. S-6: ${ }^{1} \mathrm{H}$ Spectra of 2-(3-(1H-pyrrol-1-yl)pyridin-2-yloxy)acetaldehyde 1. 


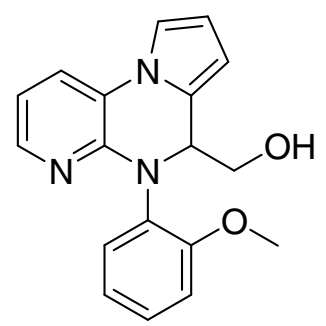

$\mathrm{C}_{18} \mathrm{H}_{17} \mathrm{~N}_{3} \mathrm{O}_{2}$

Exact Mass: 307.13

3a Mol. Wt.: 307.35
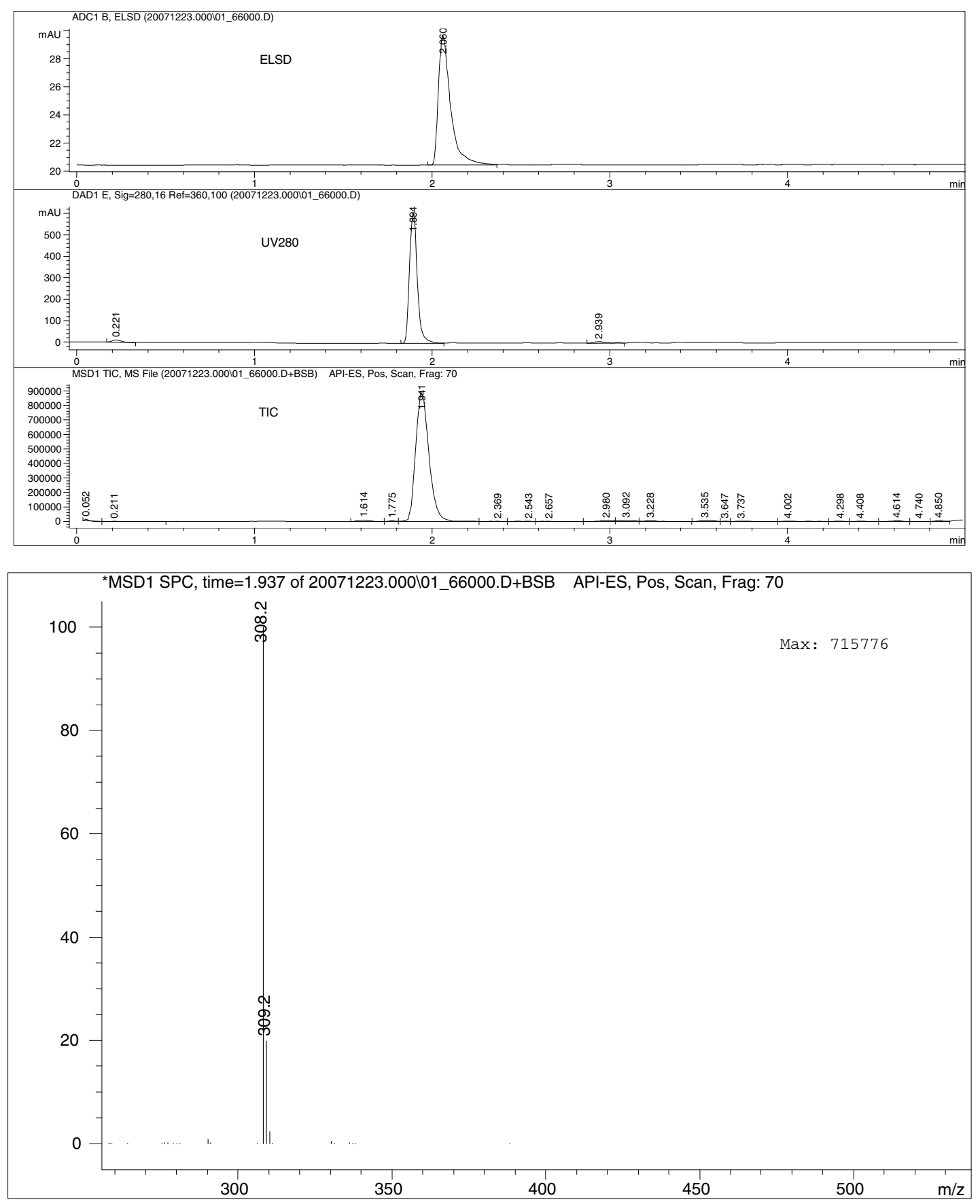

Fig. S-7: LC-MS-ELSD of 5,6-dihydro-6-hydroxymethyl-5-(2-methoxyphenyl) pyrido[2,3-e $]$ pyrrolo[1,2-a]pyrazine 3a. 


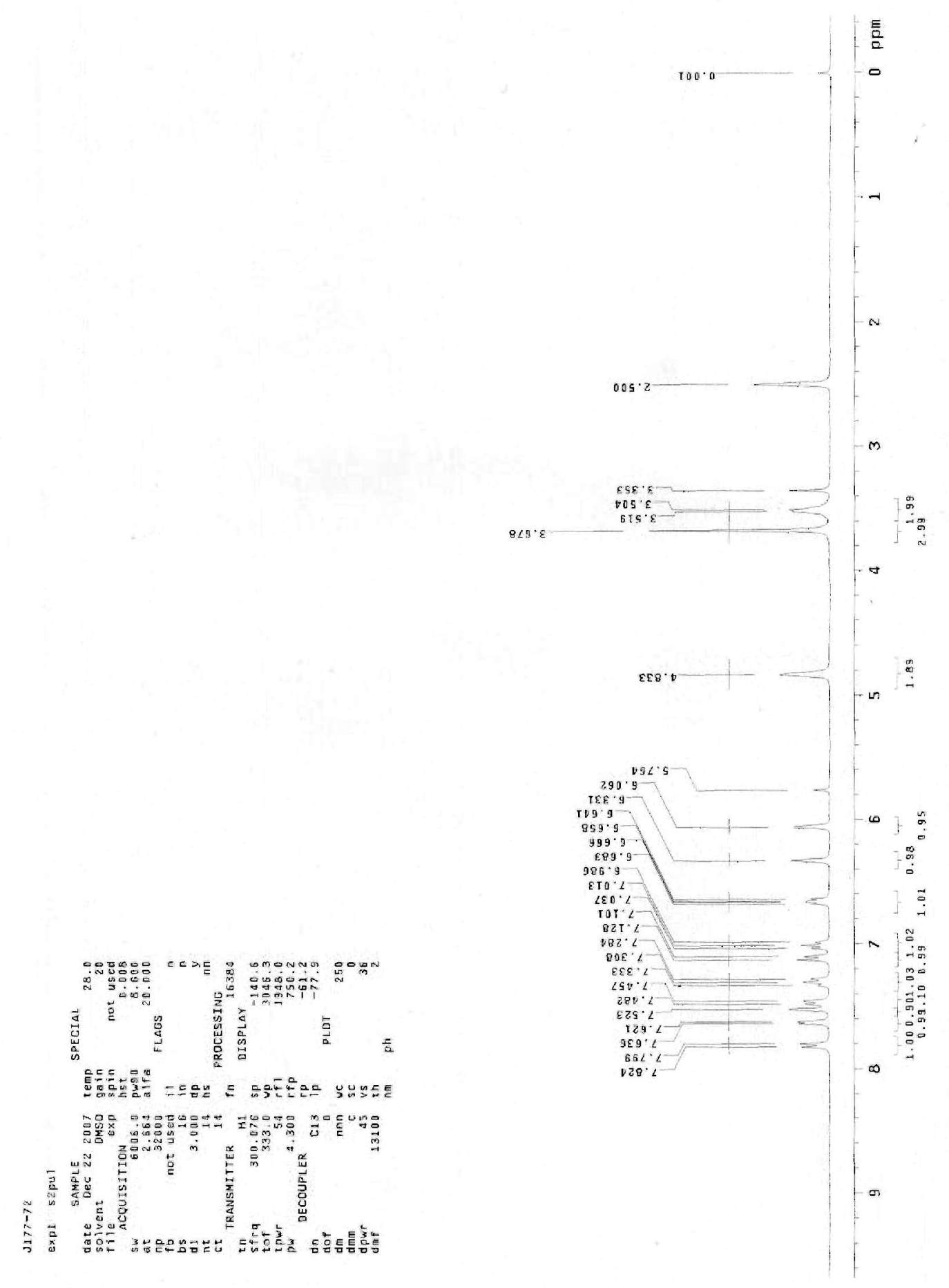

Fig. S-8: ${ }^{1} \mathrm{H}$ Spectra of 5,6-dihydro-6-hydroxymethyl-5-(2-methoxyphenyl) pyrido[2,3-e $]$ pyrrolo[1,2-a]pyrazine 3a. 


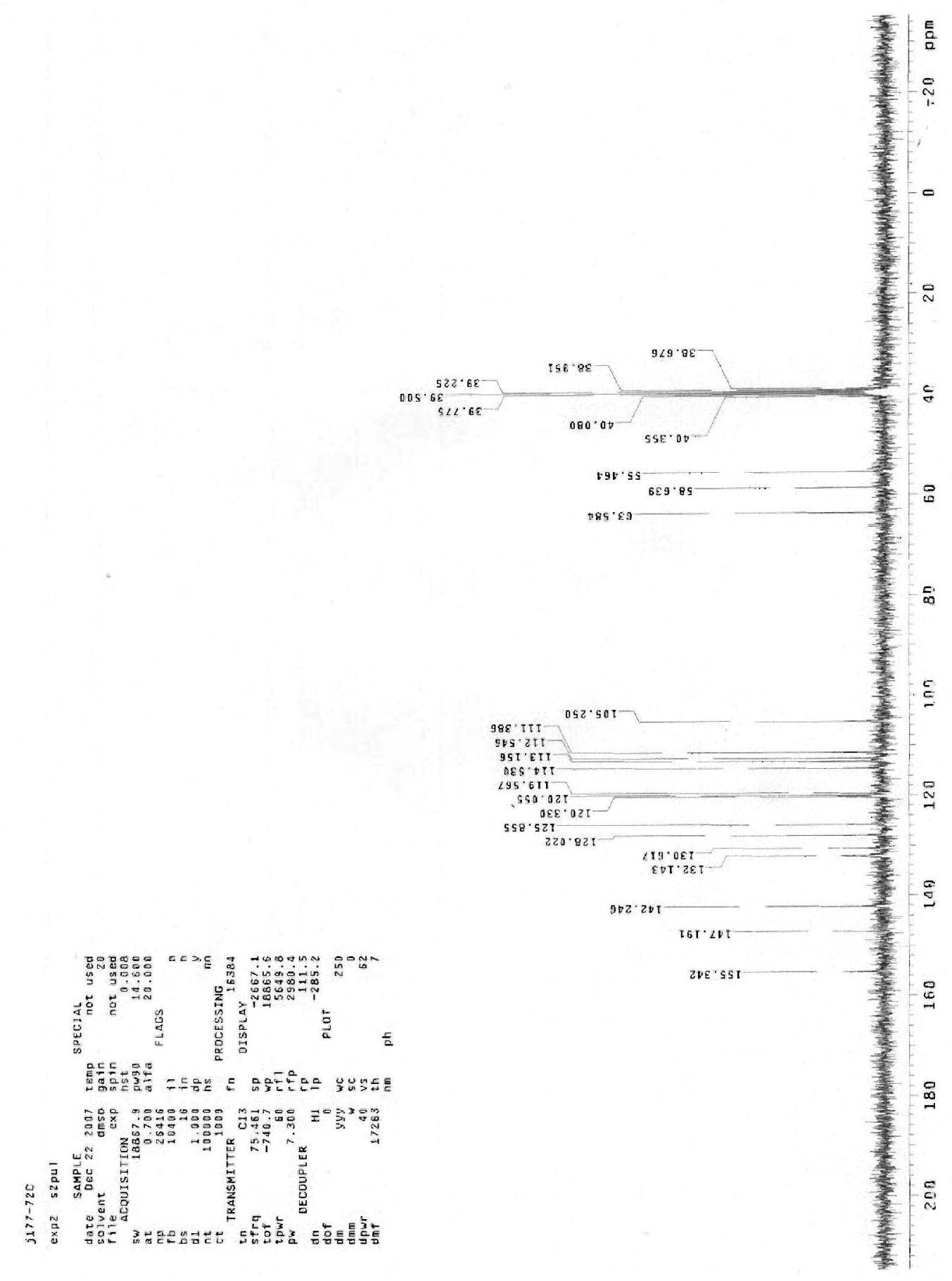

Fig. S-9: ${ }^{13} \mathrm{C}$ Spectra of 5,6-dihydro-6-hydroxymethyl-5-(2-methoxyphenyl) pyrido[2,3-e $]$ pyrrolo[1,2-a]pyrazine 3a. 


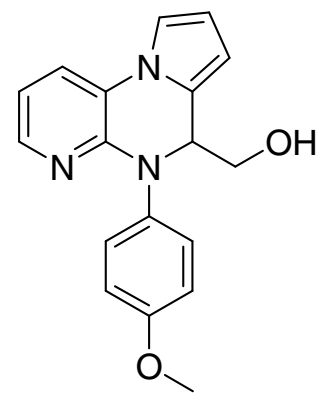

$\mathrm{C}_{18} \mathrm{H}_{17} \mathrm{~N}_{3} \mathrm{O}_{2}$

Exact Mass: 307.13

3b Mol. Wt.: 307.35
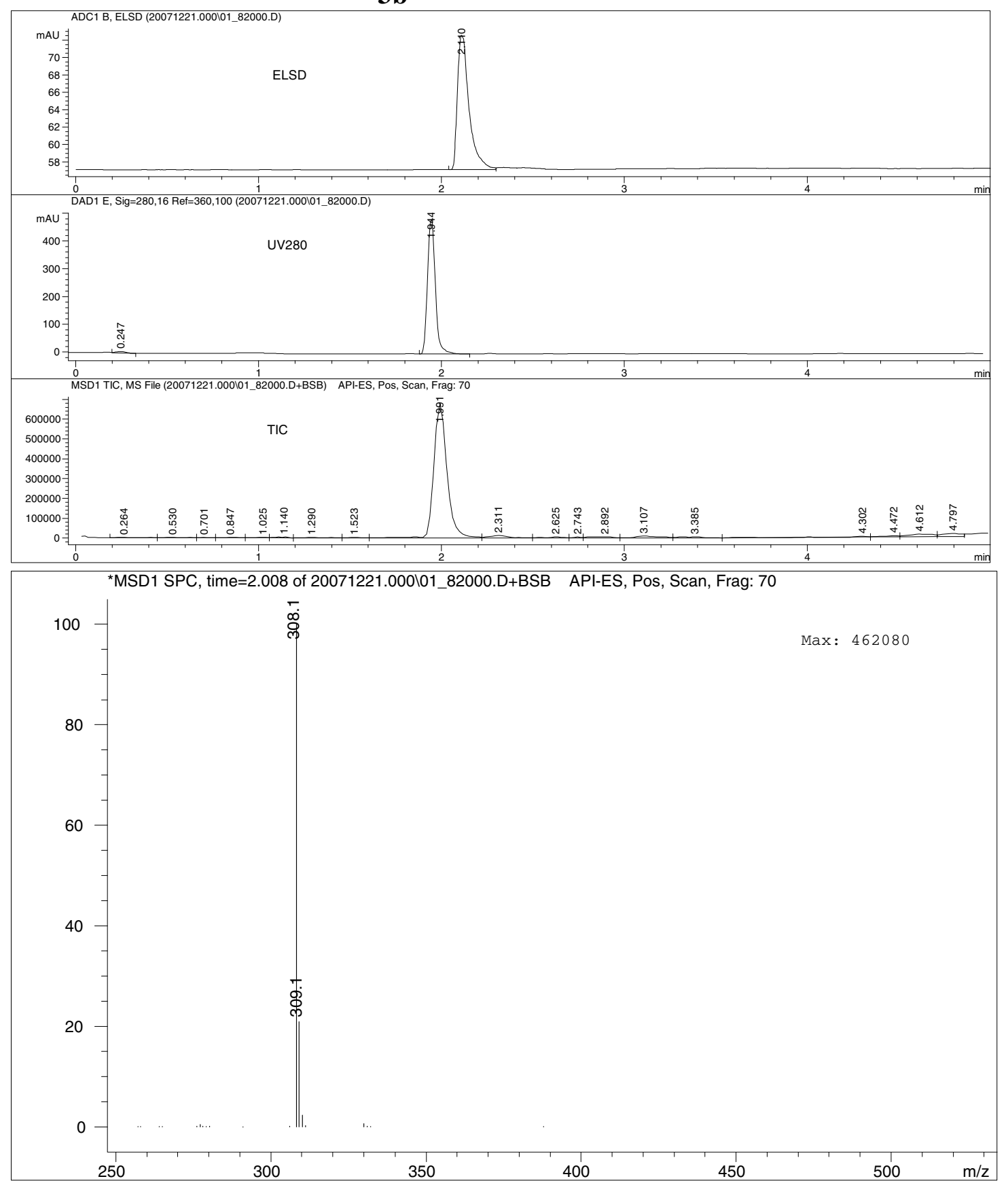

Fig. S-10: LC-MS-ELSD of 5,6-dihydro-6-hydroxymethyl-5-(4-methoxyphenyl) pyrido[2,3-e $]$ pyrrolo[1,2-a]pyrazine $3 b$. 


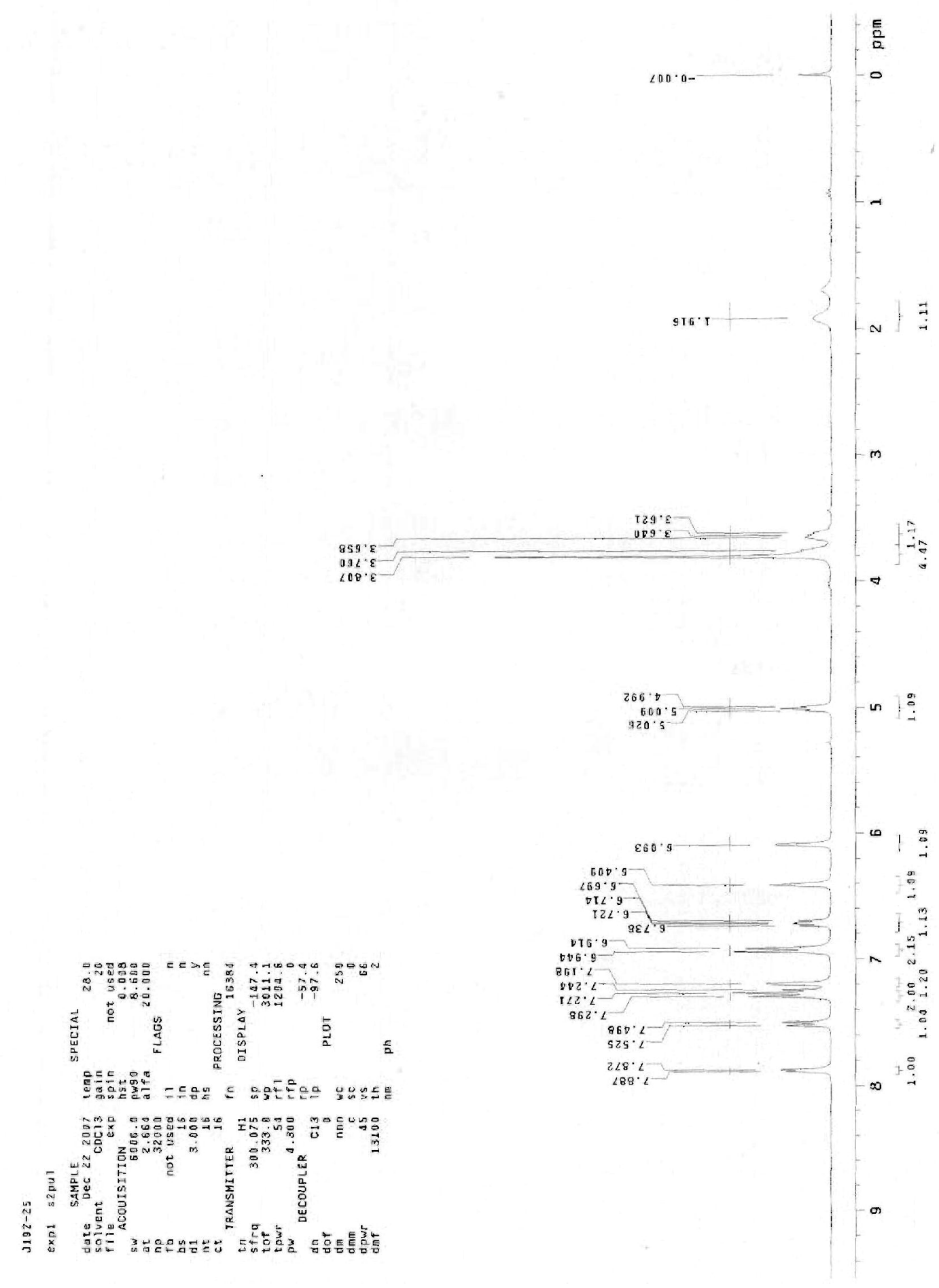

Fig. S-11: ${ }^{1} \mathrm{H}$ Spectra of 5,6-dihydro-6-hydroxymethyl-5-(4-methoxyphenyl) pyrido[2,3-e]pyrrolo[1,2-a]pyrazine $3 b$. 


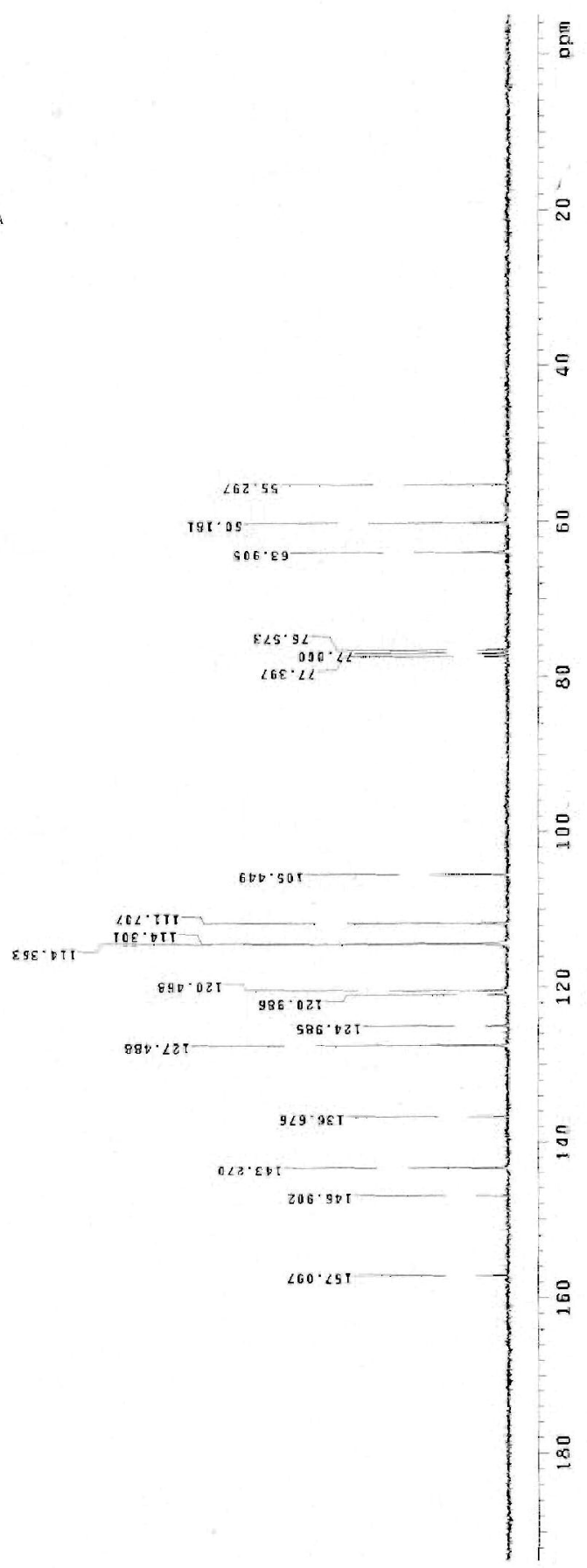

Fig. S-12: ${ }^{13} \mathrm{C}$ Spectra of 5,6-dihydro-6-hydroxymethyl-5-(4-methoxyphenyl) pyrido[2,3-e]pyrrolo[1,2-a]pyrazine $3 \mathrm{~b}$. 


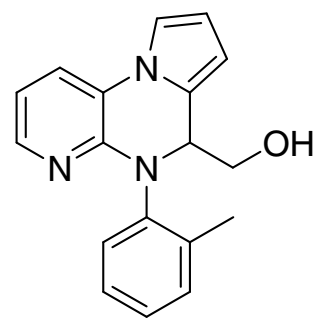

$\mathrm{C}_{18} \mathrm{H}_{17} \mathrm{~N}_{3} \mathrm{O}$

Exact Mass: 291.14

3c Mol. Wt.: 291.35
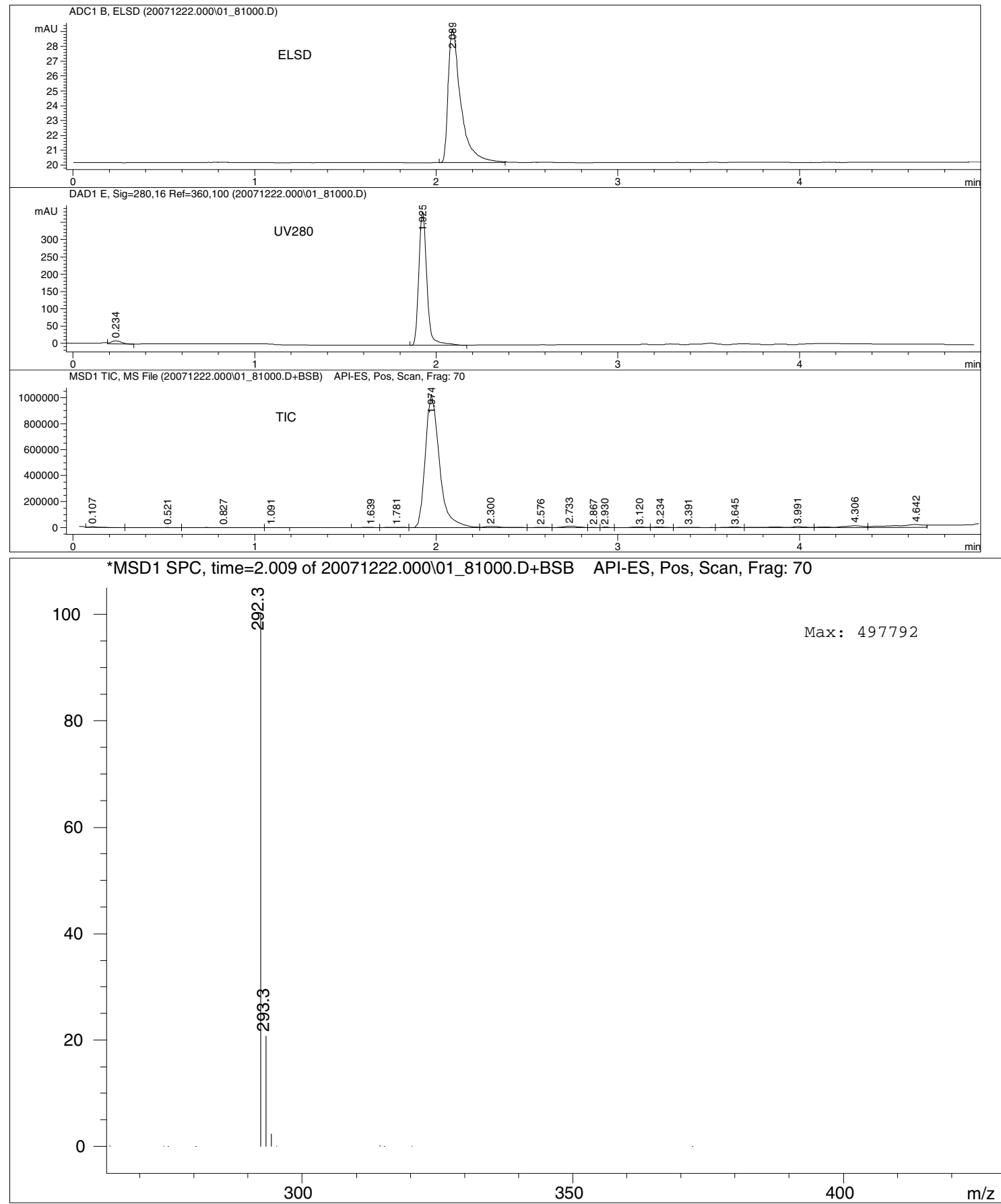

Fig. S-13: LC-MS-ELSD of 5,6-dihydro-6-hydroxymethyl-5-(2-methylphenyl) pyrido[2,3-e]pyrrolo[1,2-a]pyrazine 3c. 


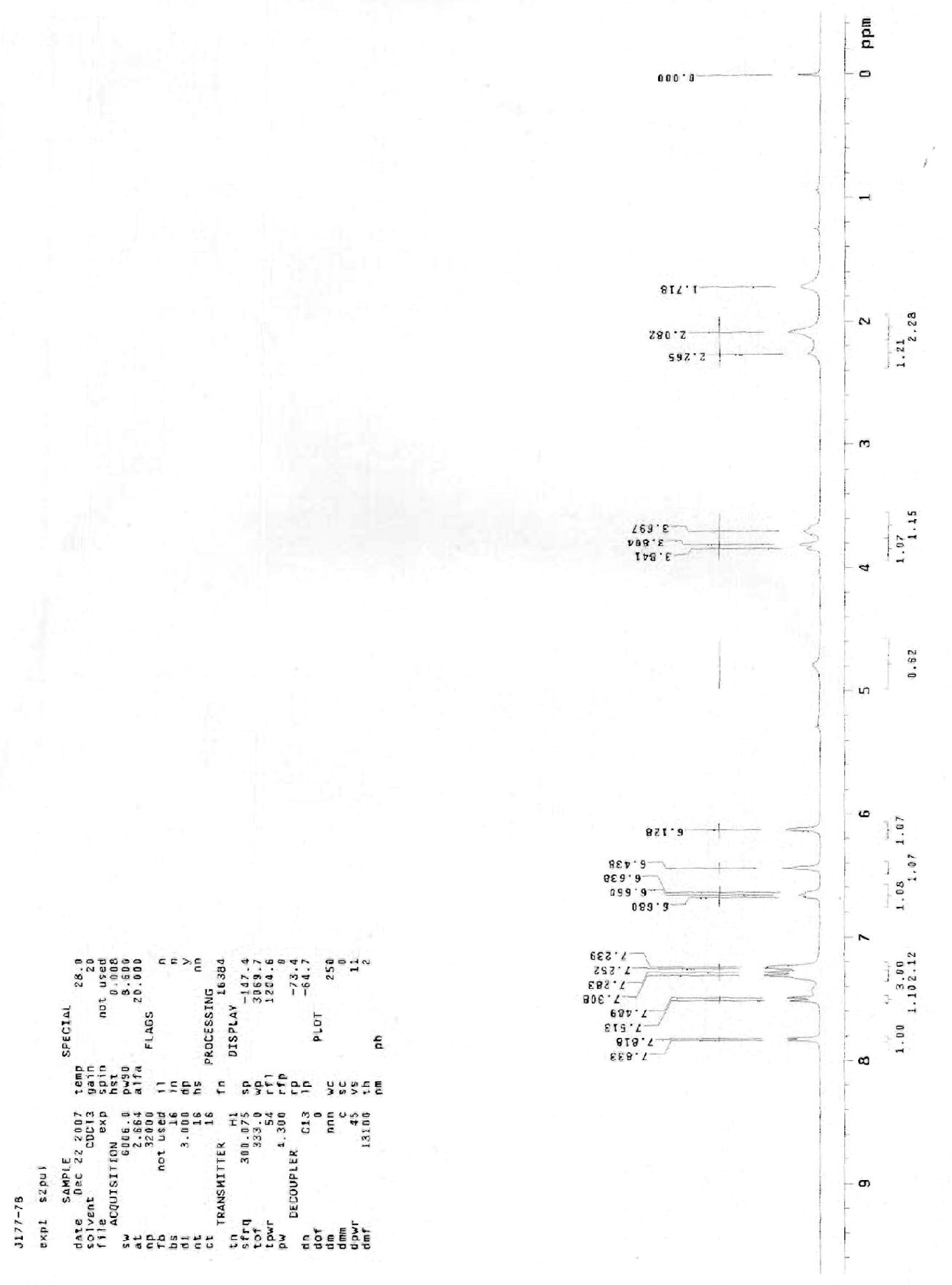

Fig. S-14: ${ }^{1} \mathrm{H}$ Spectra of 5,6-Dihydro-6-hydroxymethyl-5-(2-methylphenyl) pyrido $[2,3-e]$ pyrrolo $[1,2-a]$ pyrazine $3 \mathrm{c}$. 


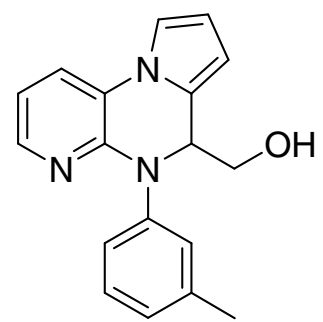

$\mathrm{C}_{18} \mathrm{H}_{17} \mathrm{~N}_{3} \mathrm{O}$

Exact Mass: 291.14

3d Mol. Wt.: 291.35
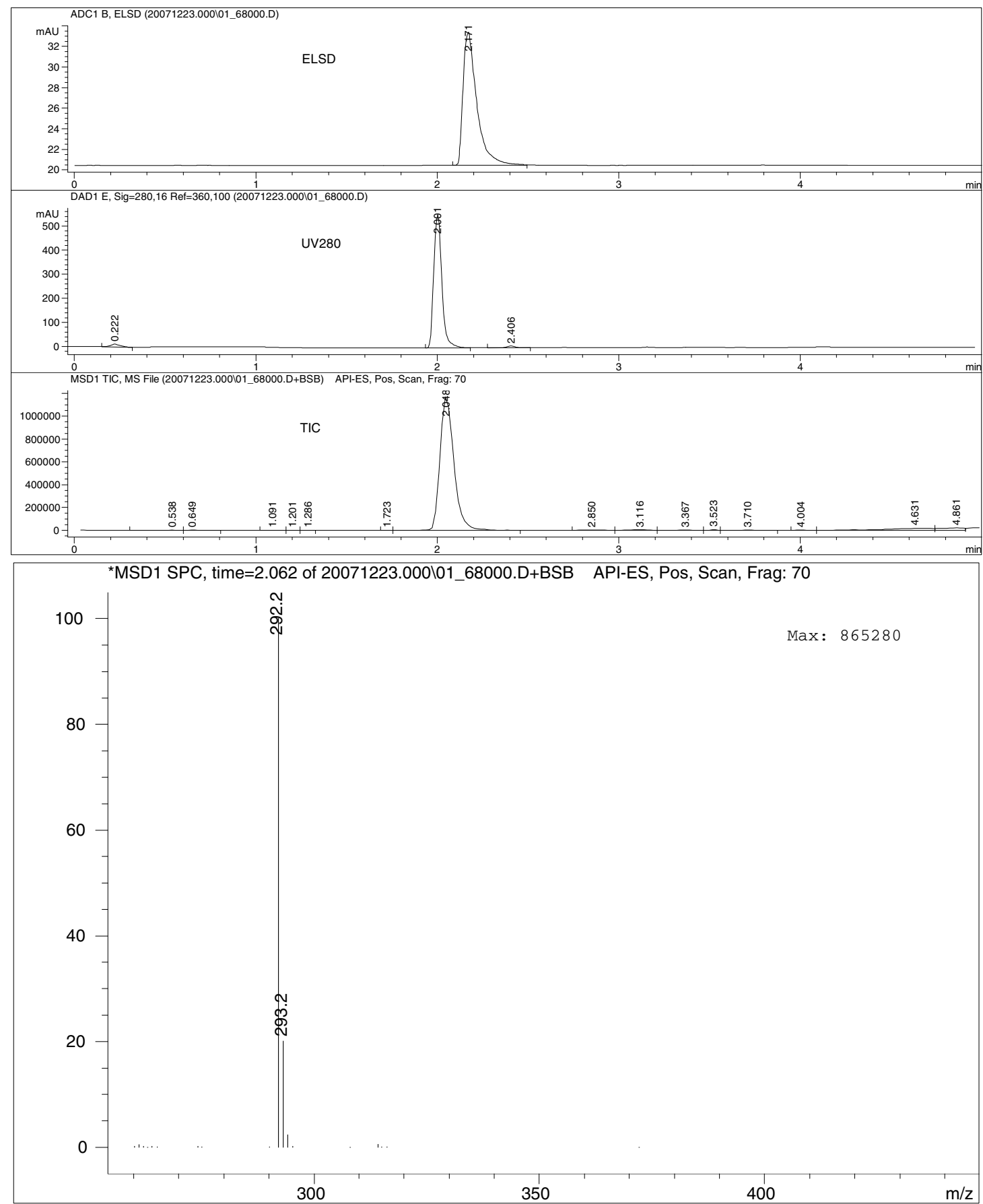

Fig. S-15: LC-MS-ELSD of 5,6-dihydro-6-hydroxymethyl-5-(3-methylphenyl) pyrido[2,3-e $]$ pyrrolo[1,2-a]pyrazine $3 \mathrm{~d}$. 


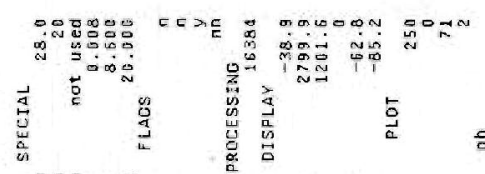

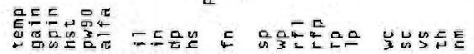

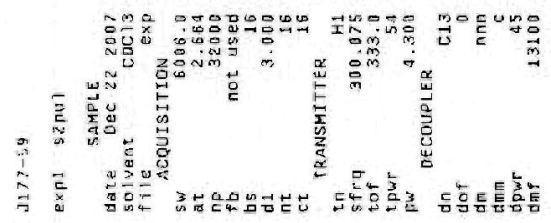

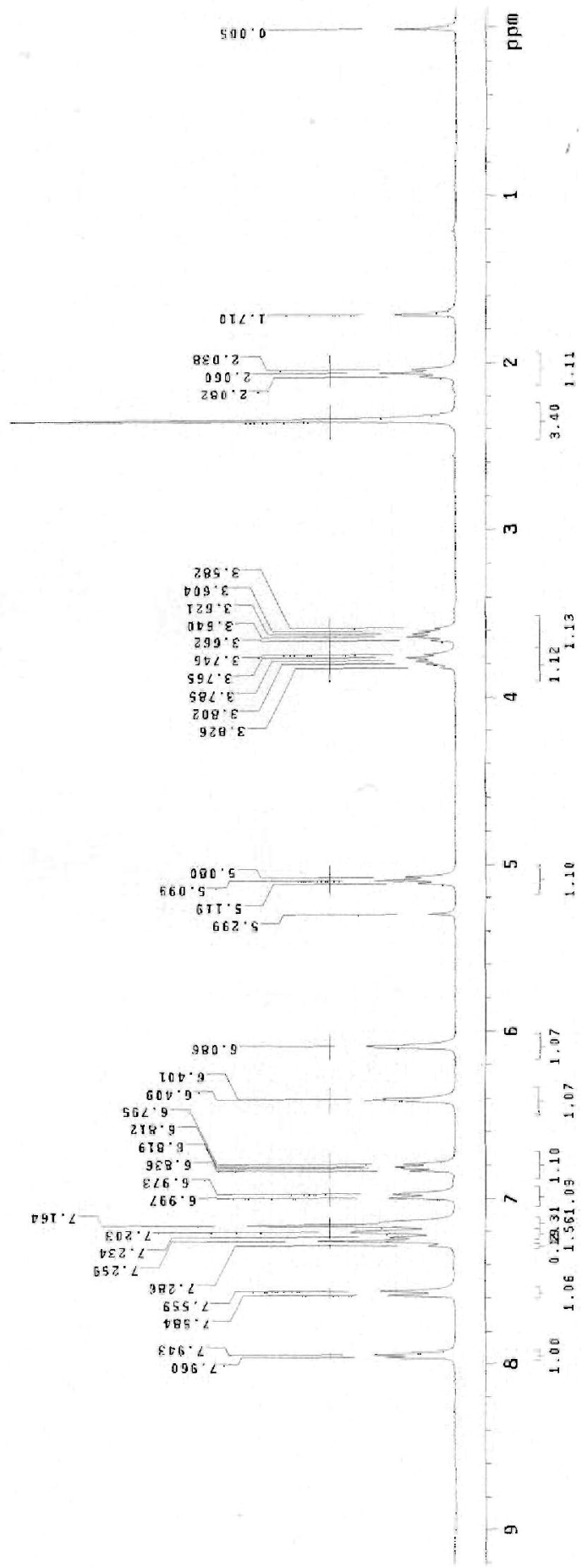

Fig. S-16: ${ }^{1} \mathrm{H}$ Spectra of 5,6-dihydro-6-hydroxymethyl-5-(3-methylphenyl) pyrido[2,3-e]pyrrolo[1,2-a]pyrazine 3d. 

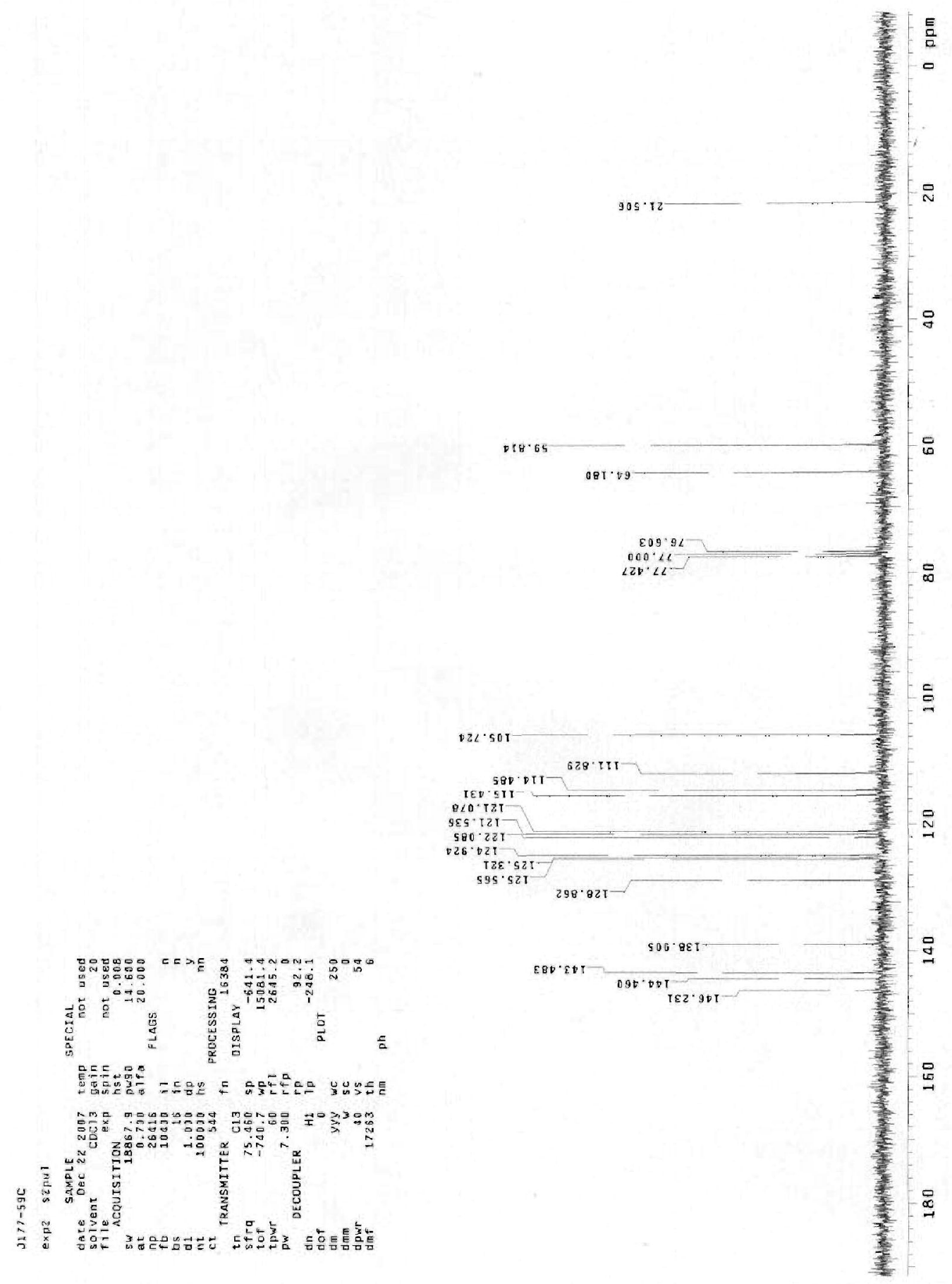

Fig. S-17: ${ }^{13} \mathrm{C}$ Spectra of 5,6-dihydro-6-hydroxymethyl-5-(3-methylphenyl) pyrido[2,3-e]pyrrolo[1,2-a]pyrazine 3d. 


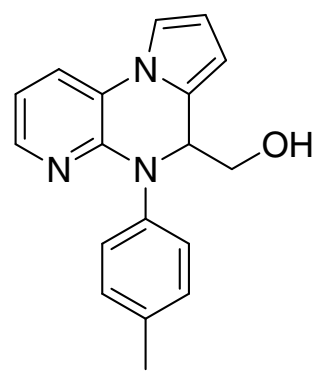

$\mathrm{C}_{18} \mathrm{H}_{17} \mathrm{~N}_{3} \mathrm{O}$

Exact Mass: 291.14

3e Mol. Wt.: 291.35

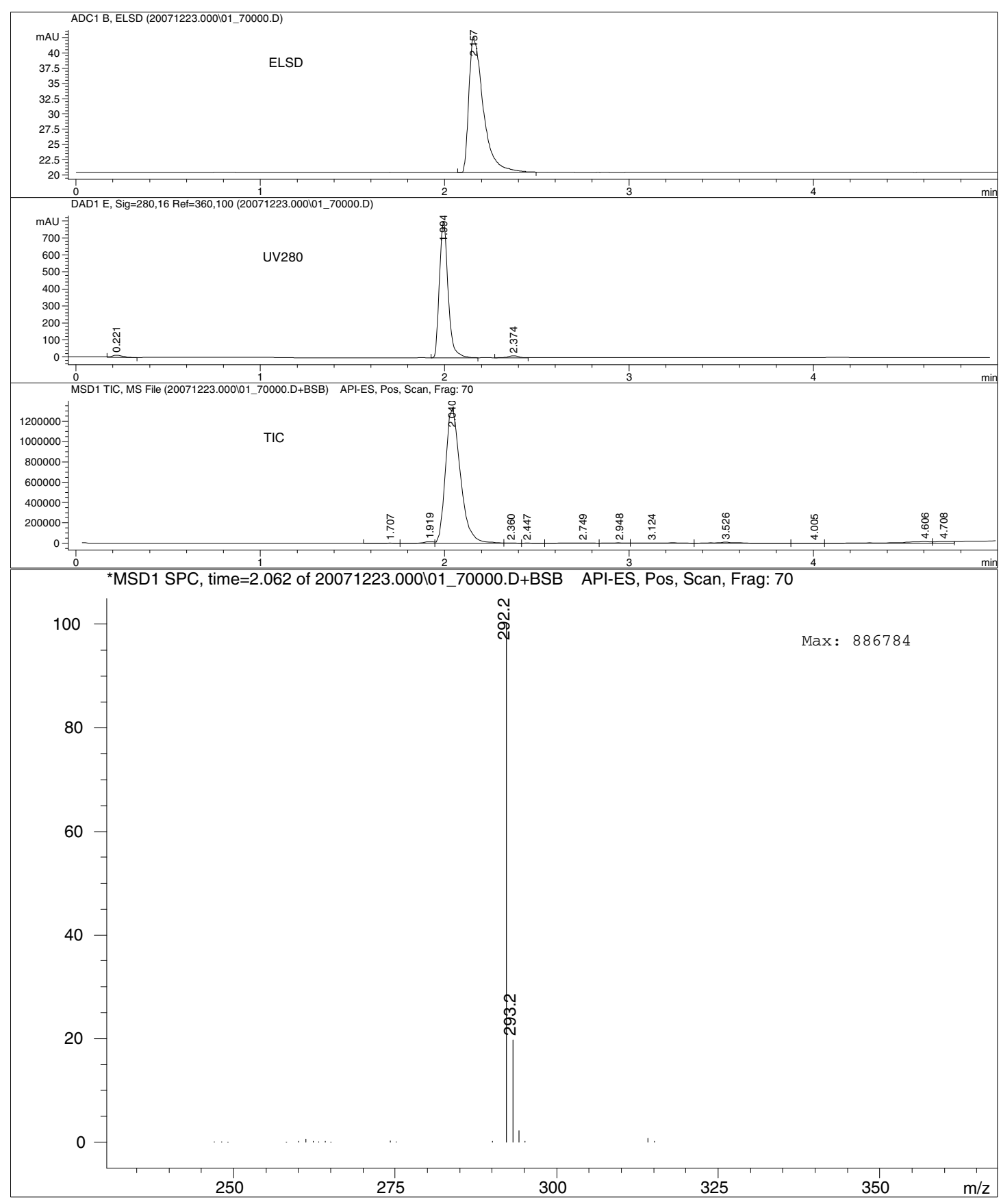

Fig. S-18: LC-MS-ELSD of 5,6-dihydro-6-hydroxymethyl-5-(4-methylphenyl) pyrido[2,3-e]pyrrolo[1,2-a]pyrazine 3e. 


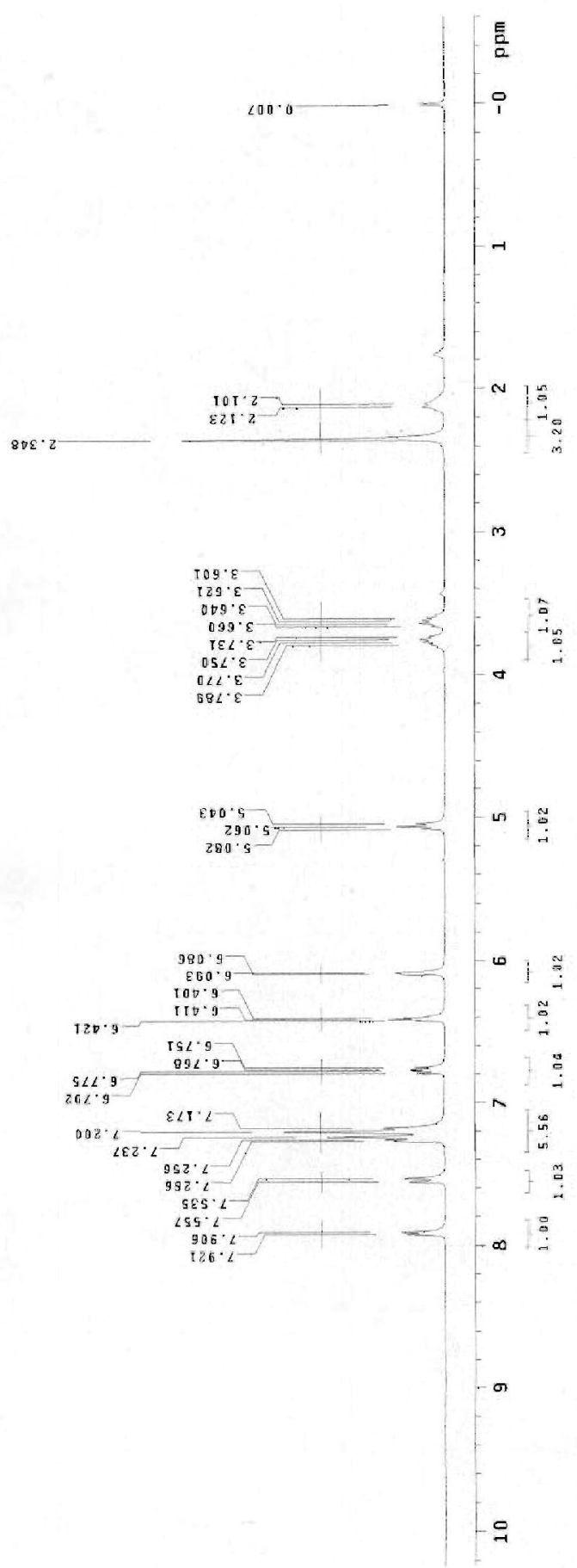

Fig. S-19: ${ }^{1} \mathrm{H}$ Spectra of 5,6-dihydro-6-hydroxymethyl-5-(4-methylphenyl) pyrido[2,3-e]pyrrolo[1,2-a]pyrazine $3 \mathrm{e}$. 


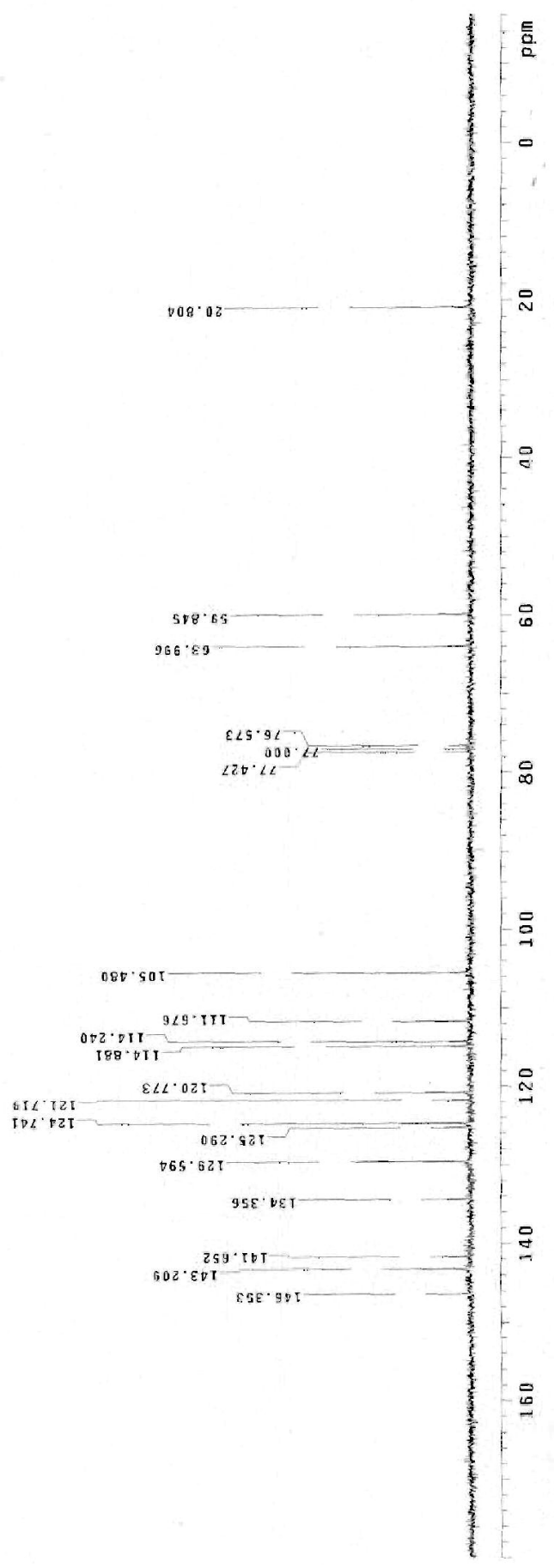

Fig. S-20: ${ }^{13} \mathrm{C}$ Spectra of 5,6-dihydro-6-hydroxymethyl-5-(4-methylphenyl) pyrido $[2,3-e]$ pyrrolo $[1,2-a]$ pyrazine $3 \mathrm{e}$. 


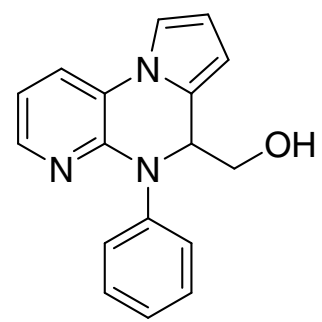

$\mathrm{C}_{17} \mathrm{H}_{15} \mathrm{~N}_{3} \mathrm{O}$

Exact Mass: 277.12

3f Mol. Wt.: 277.32

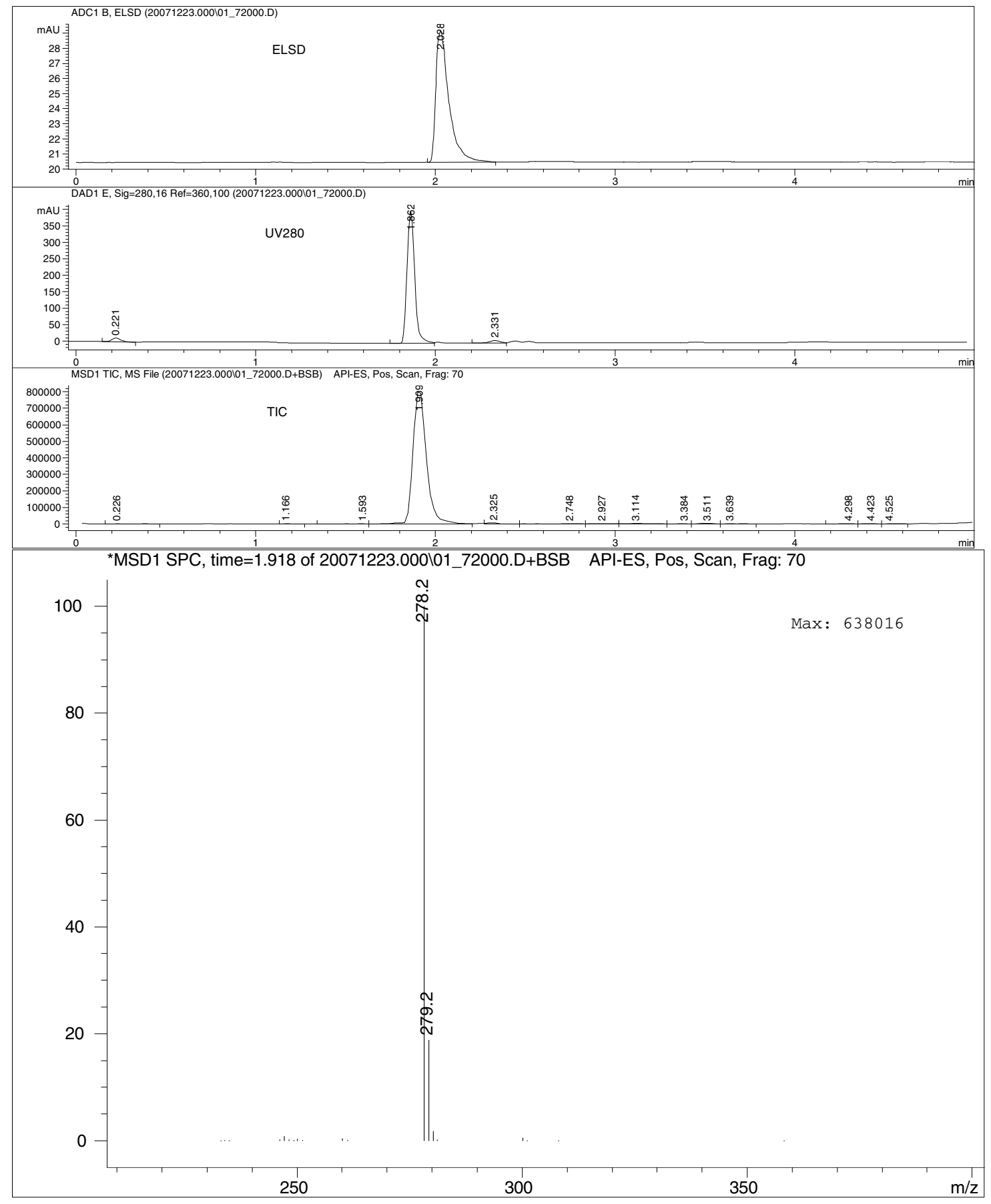

Fig. S-21: LC-MS-ELSD of 5,6-dihydro-6-hydroxymethyl-5-phenylpyrido[2,3-e] pyrrolo[1,2-a]pyrazine $3 f$. 


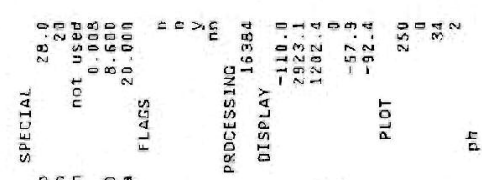

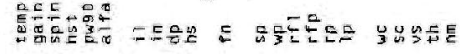

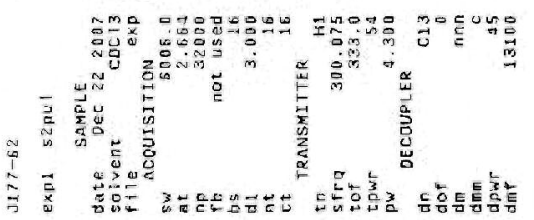

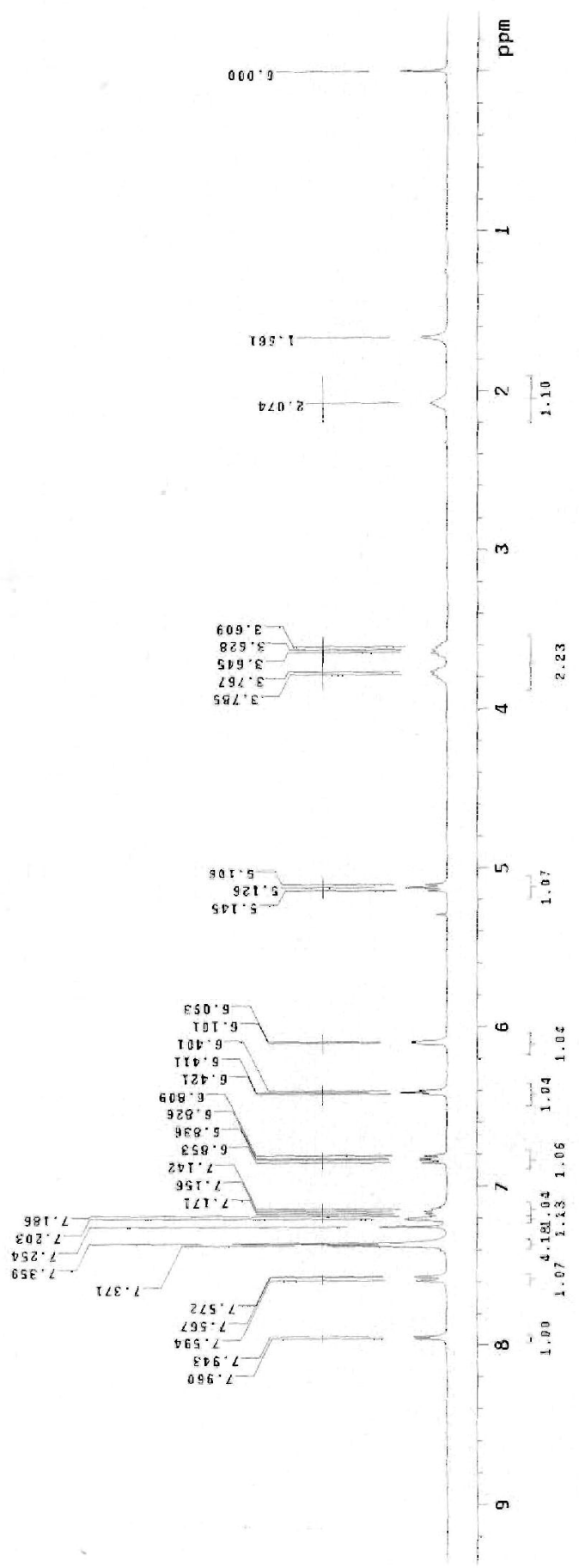

Fig. S-22: ${ }^{1} \mathrm{H}$ Spectra of 5,6-dihydro-6-hydroxymethyl-5-phenylpyrido[2,3-e $]$ pyrrolo[1,2-a]pyrazine $3 f$. 

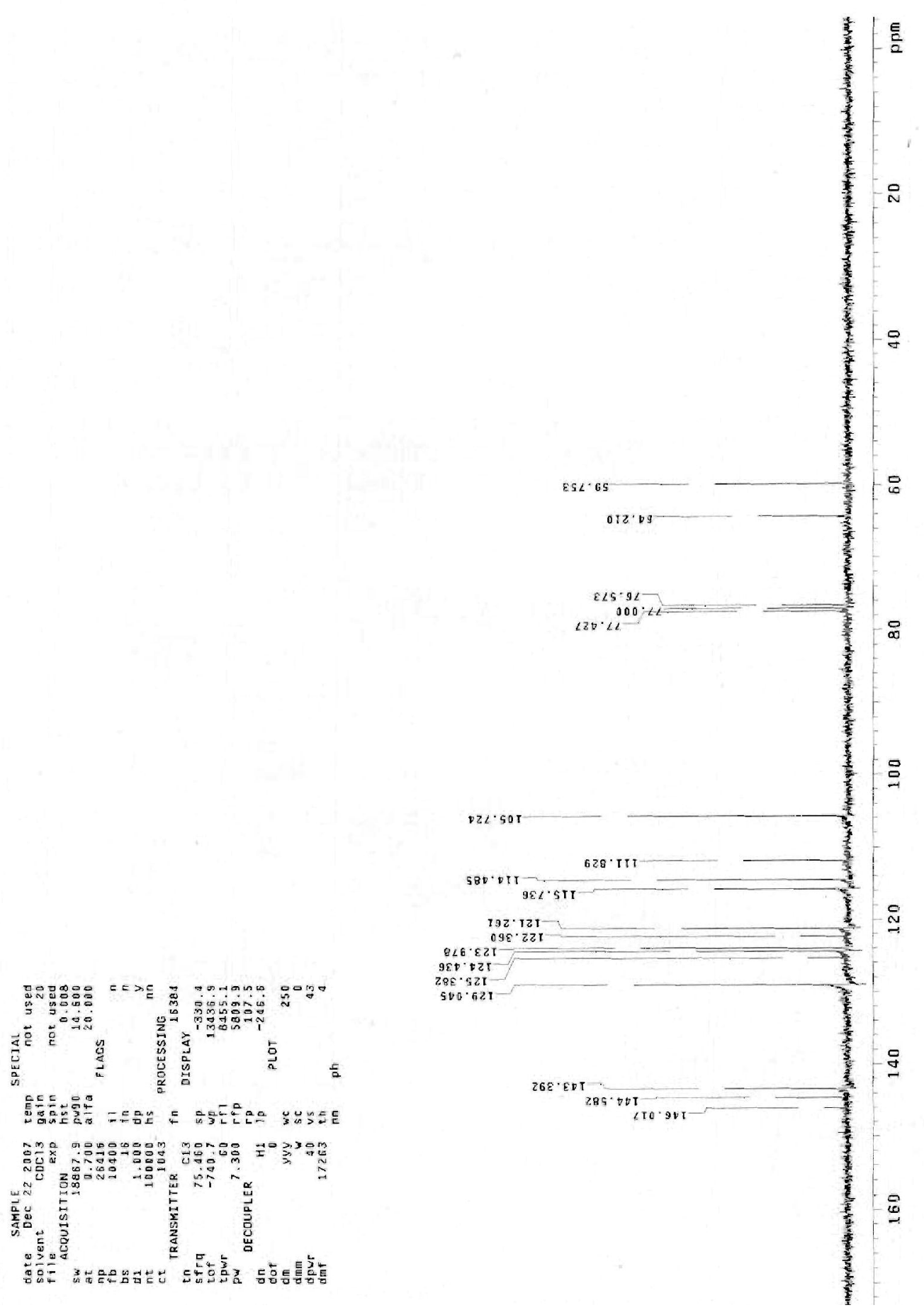

Fig. S-23: ${ }^{13} \mathrm{C}$ Spectra of 5,6-dihydro-6-hydroxymethyl-5-phenylpyrido[2,3-e] pyrrolo[1,2-a]pyrazine $3 f$. 


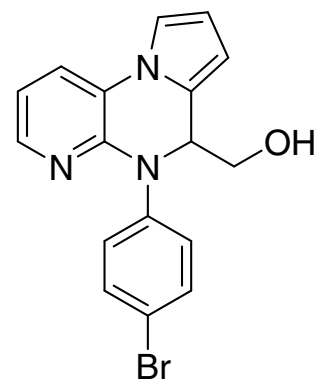

$\mathrm{C}_{17} \mathrm{H}_{14} \mathrm{BrN}_{3} \mathrm{O}$

Exact Mass: 355.03

$3 g$ Mol. Wt.: 356.22

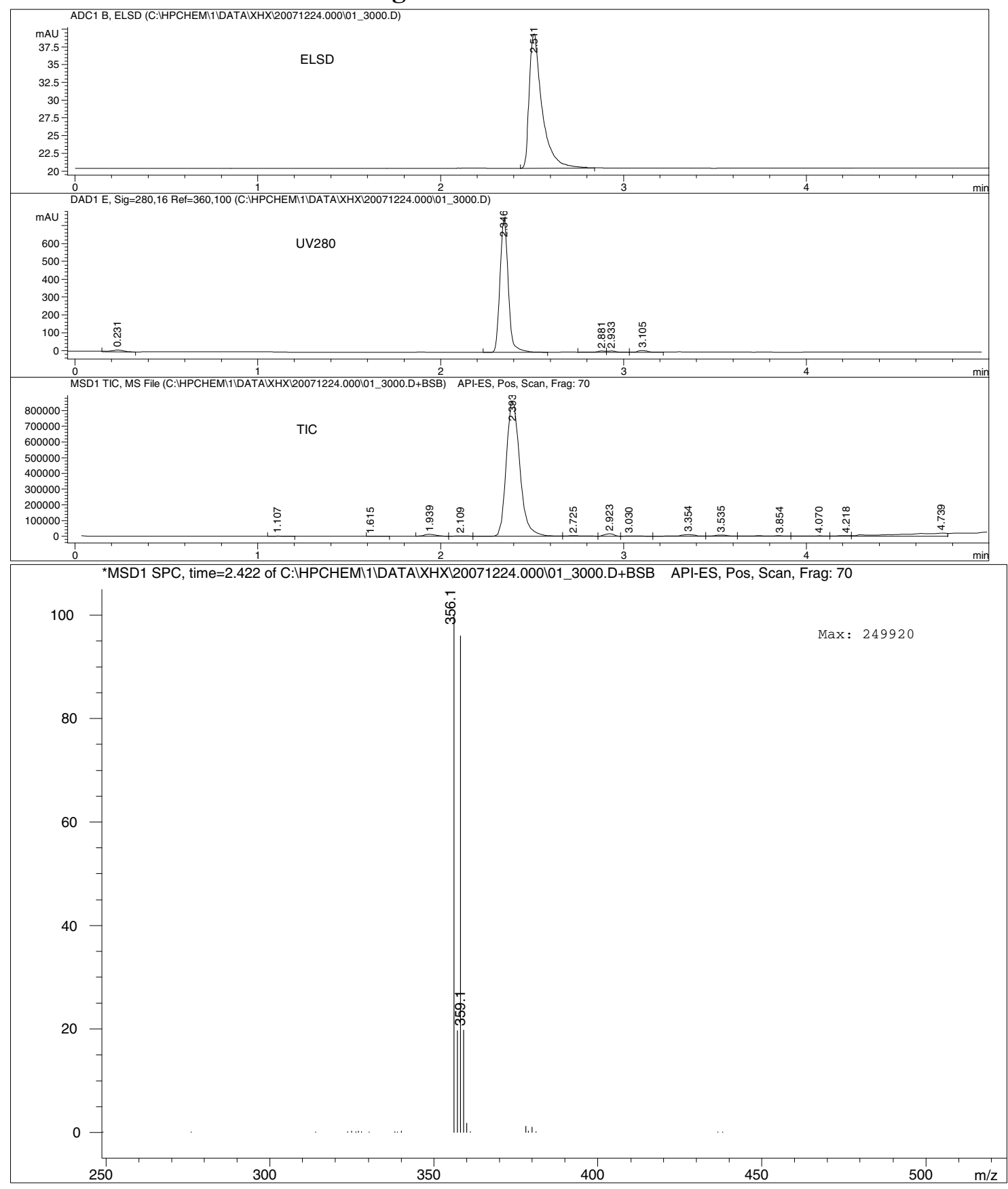

Fig. S-24: LC-MS-ELSD of 5,6-dihydro-6-hydroxymethyl-5-(4-bromophenyl) pyrido[2,3-e $]$ pyrrolo[1,2-a]pyrazine $3 \mathrm{~g}$. 


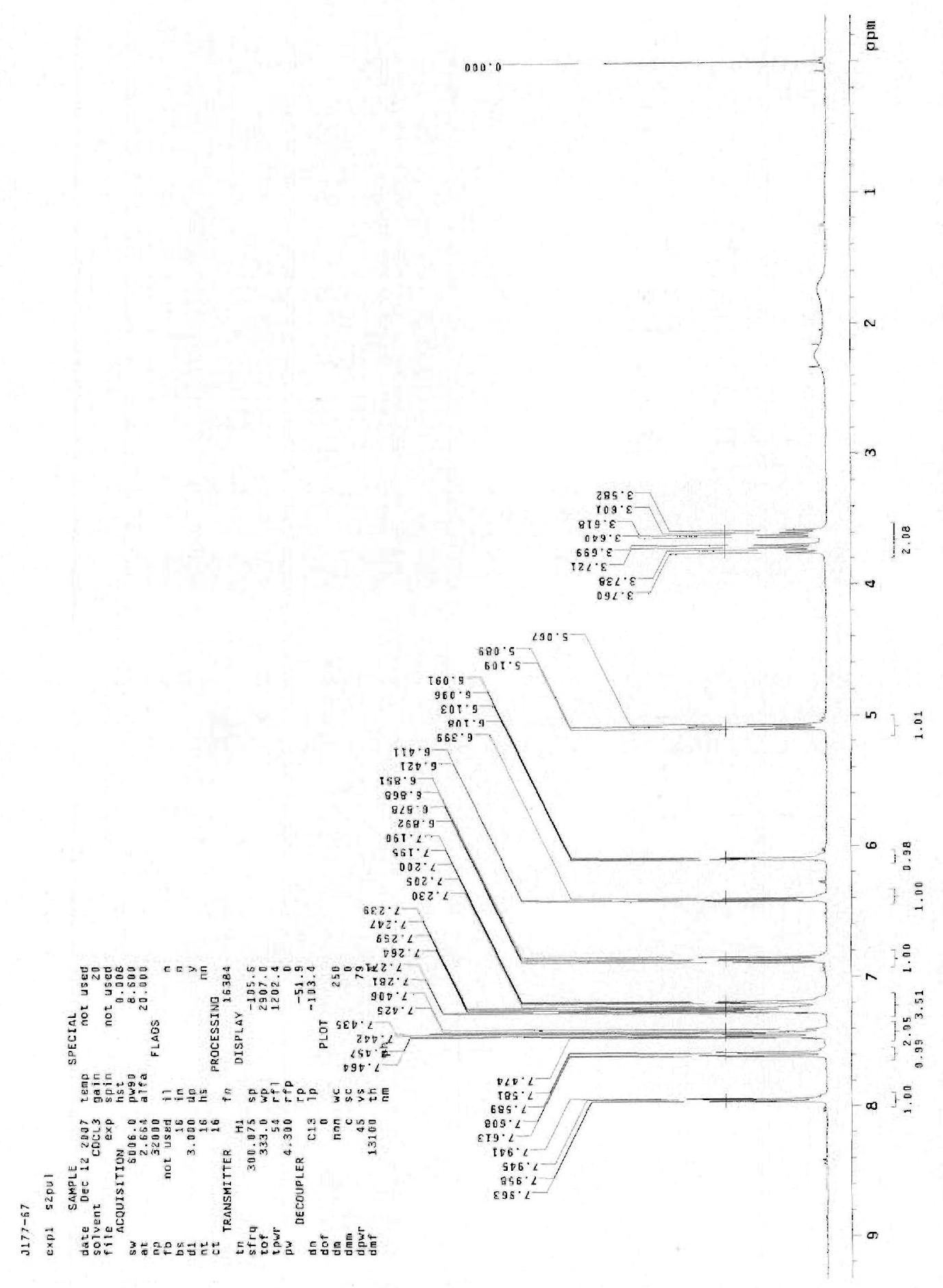

Fig. S-25: ${ }^{1} \mathrm{H}$ Spectra of 5,6-dihydro-6-hydroxymethyl-5-(4-bromophenyl) pyrido[2,3-e $]$ pyrrolo[1,2-a]pyrazine 3g. 


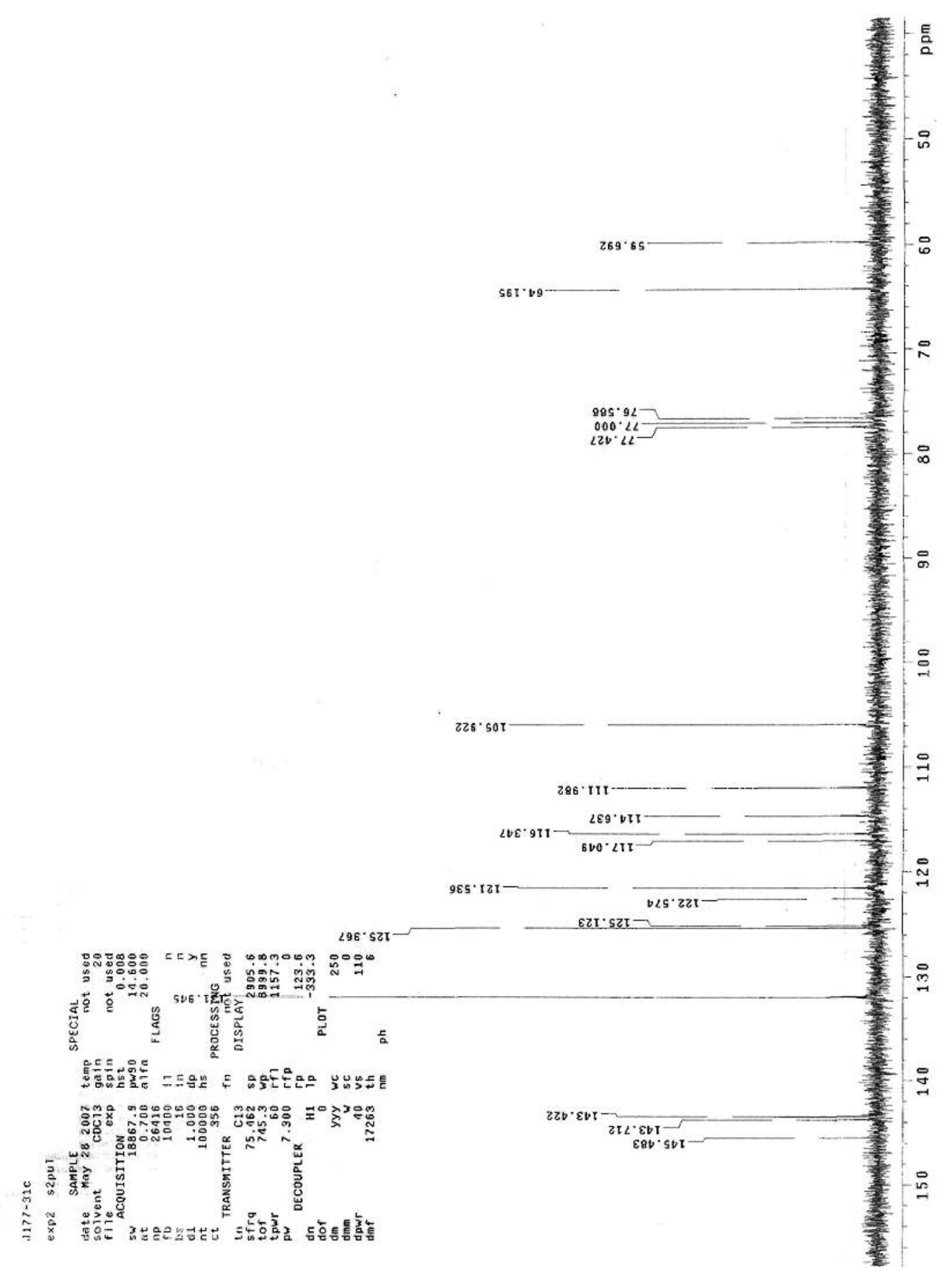

Fig. S-26: ${ }^{13} \mathrm{C}$ Spectra of 5,6-dihydro-6-hydroxymethyl-5-(4-bromophenyl) pyrido[2,3-e $]$ pyrrolo[1,2-a]pyrazine $3 \mathrm{~g}$. 


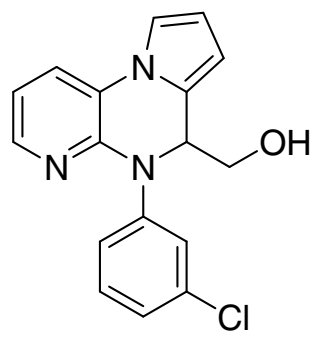

$\mathrm{C}_{17} \mathrm{H}_{14} \mathrm{ClN}_{3} \mathrm{O}$

Exact Mass: 311.08

3i Mol. Wt.: 311.77

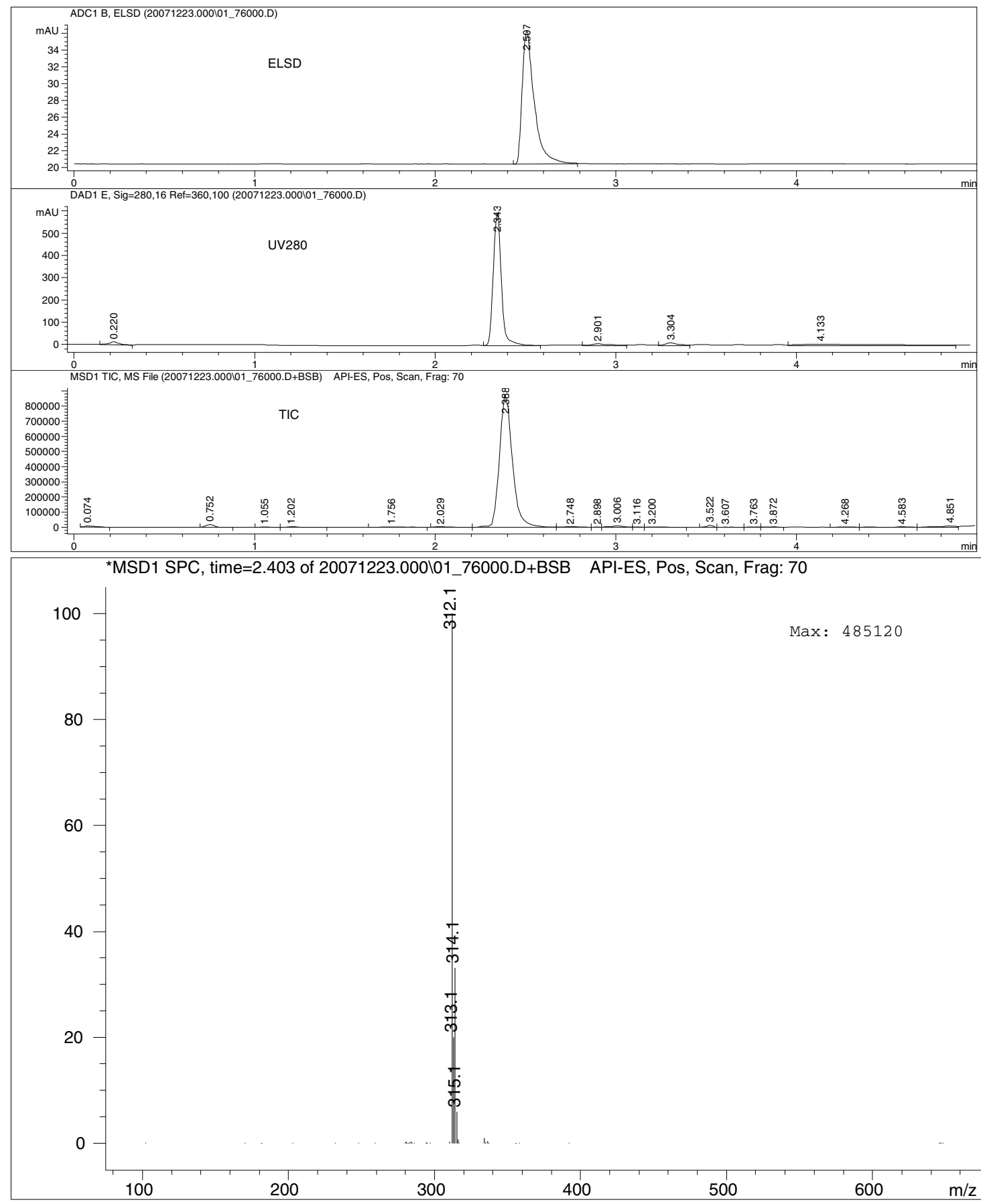

Fig. S-27: LC-MS-ELSD of 5,6-dihydro-6-hydroxymethyl-5-(3-chlorophenyl) pyrido[2,3-e]pyrrolo[1,2-a]pyrazine 3i. 


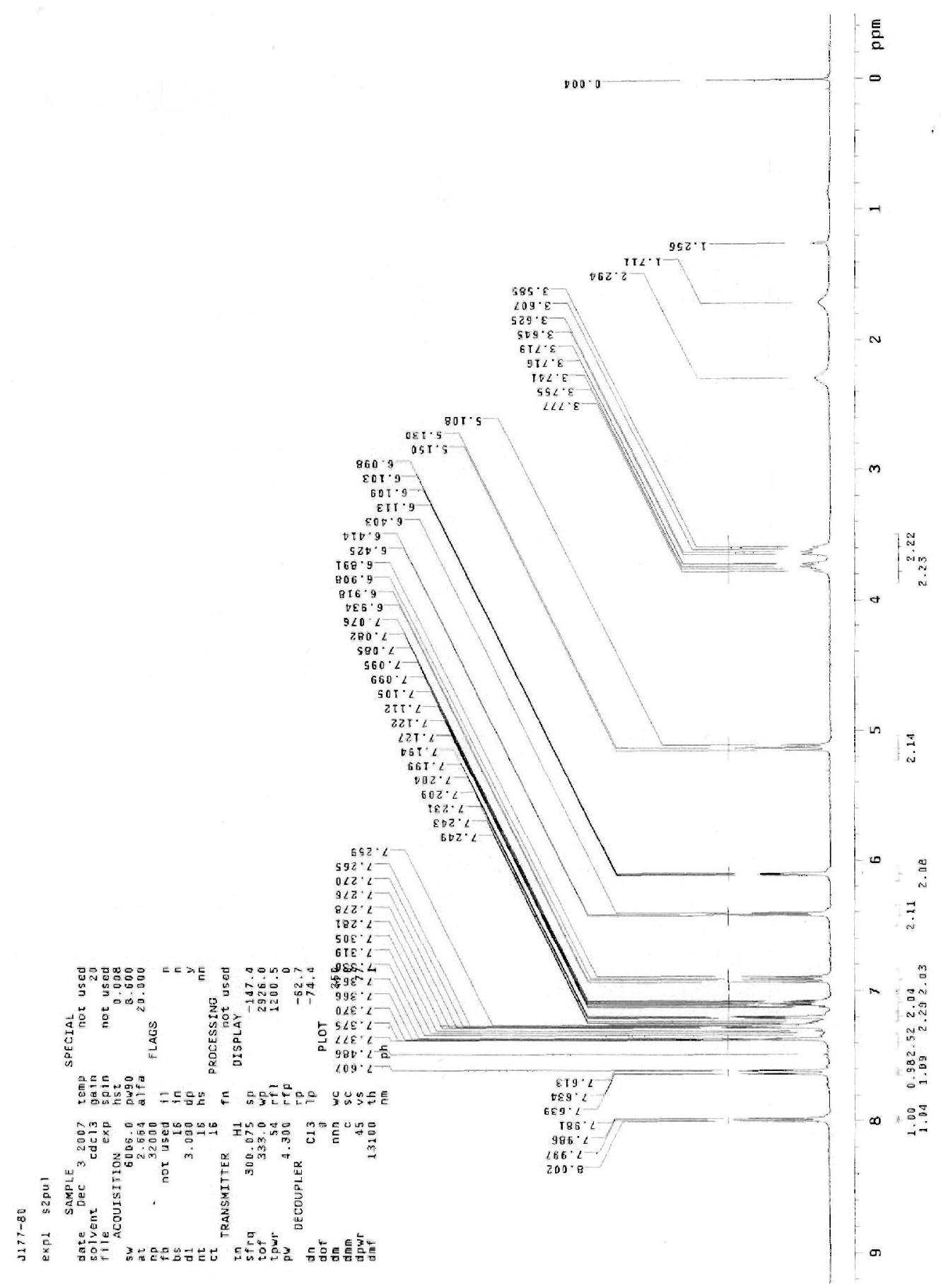

Fig. S-28: ${ }^{1} \mathrm{H}$ Spectra of 5,6-dihydro-6-hydroxymethyl-5-(3-chlorophenyl) pyrido[2,3-e $]$ pyrrolo[1,2-a]pyrazine $3 i$. 


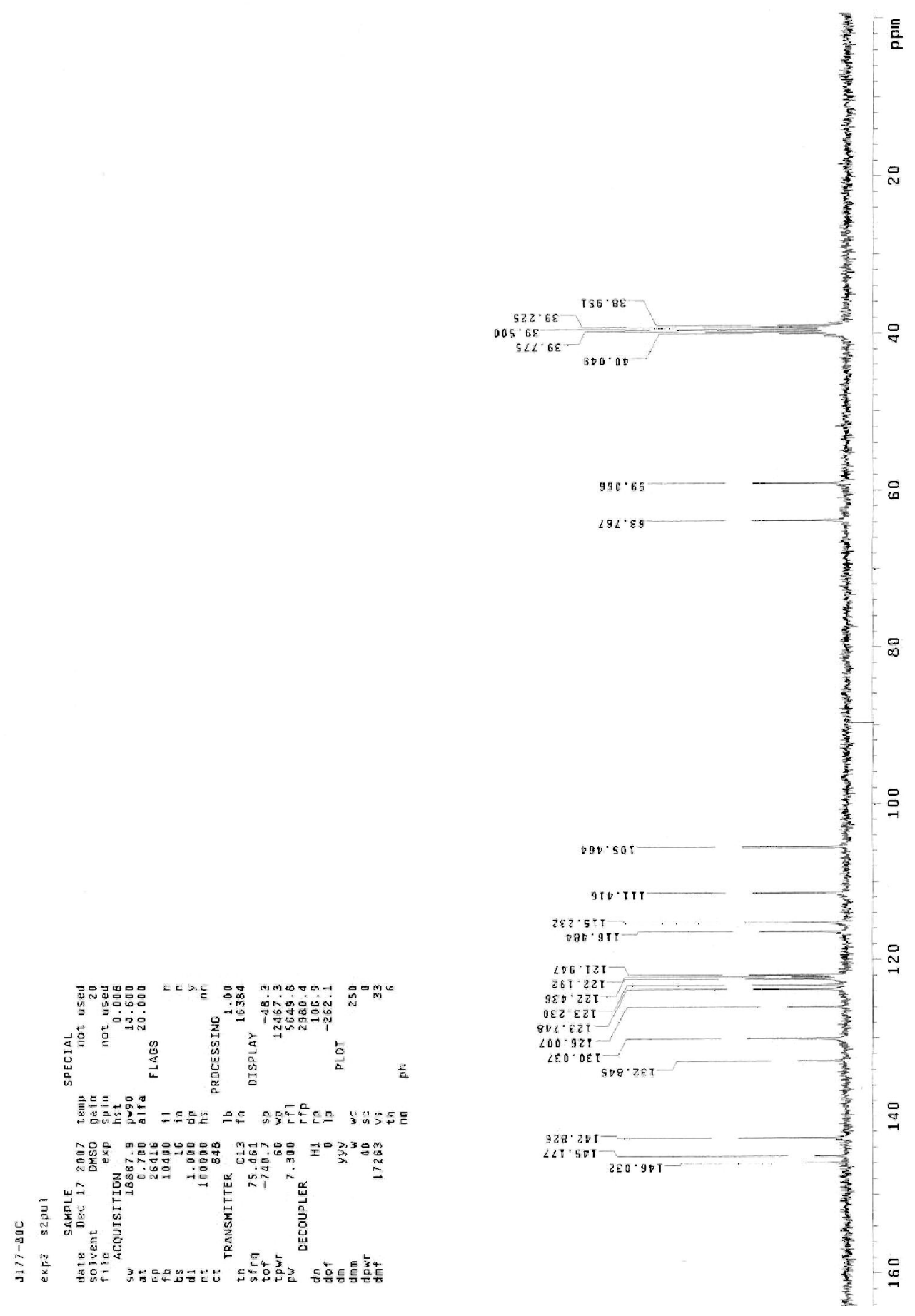

Fig. S-29: ${ }^{13} \mathrm{C}$ Spectra of 5,6-dihydro-6-hydroxymethyl-5-(3-chlorophenyl) pyrido[2,3-e]pyrrolo[1,2-a]pyrazine $3 i$. 


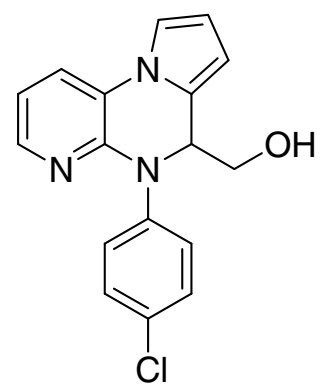

$\mathrm{C}_{17} \mathrm{H}_{14} \mathrm{CIN}_{3} \mathrm{O}$

Exact Mass: 311.08

3j Mol. Wt.: 311.77

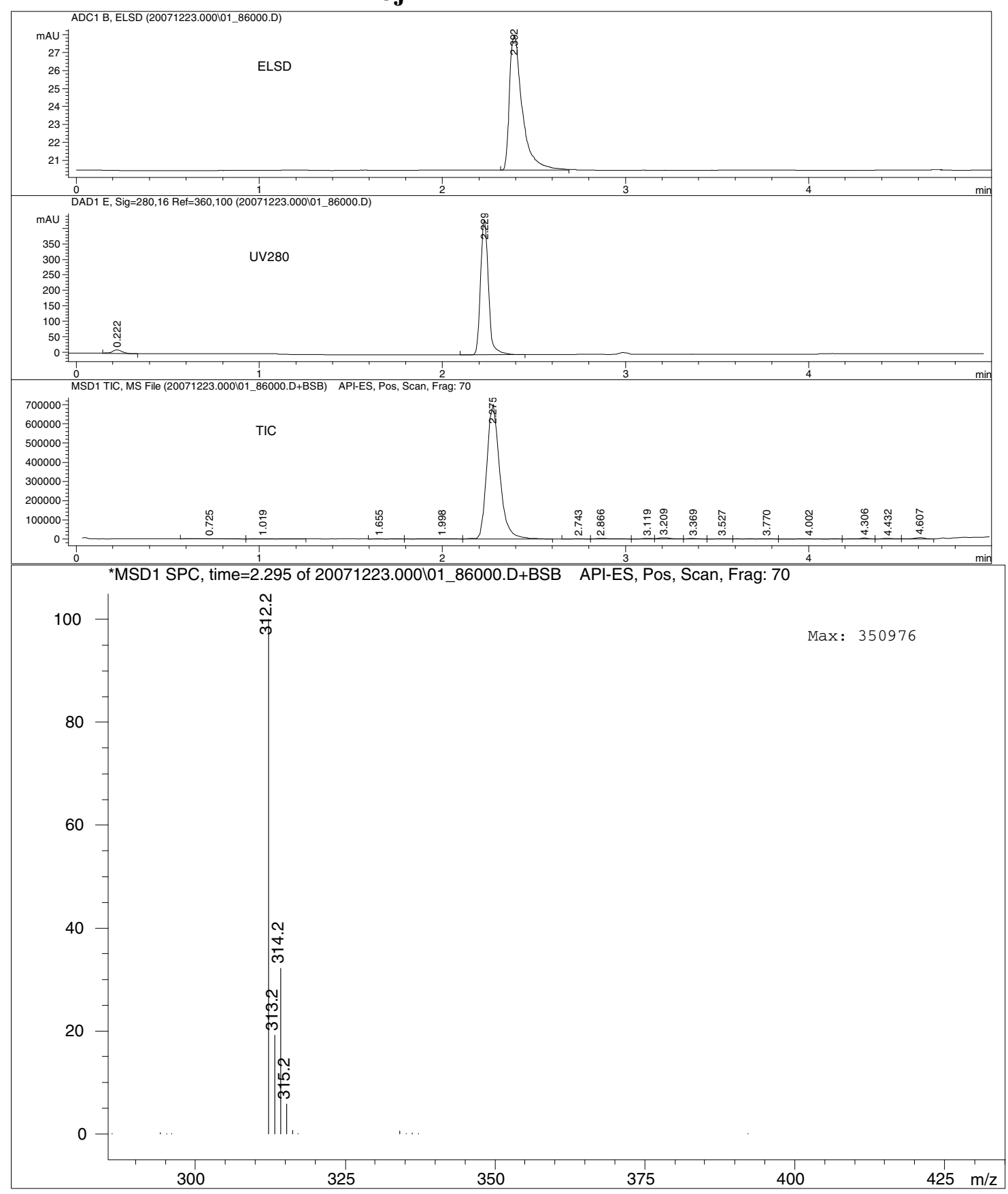

Fig. S-30: LC-MS-ELSD of 5,6-dihydro-6-hydroxymethyl-5-(4-chlorophenyl) pyrido[2,3-e]pyrrolo[1,2-a]pyrazine $3 \mathbf{j}$. 


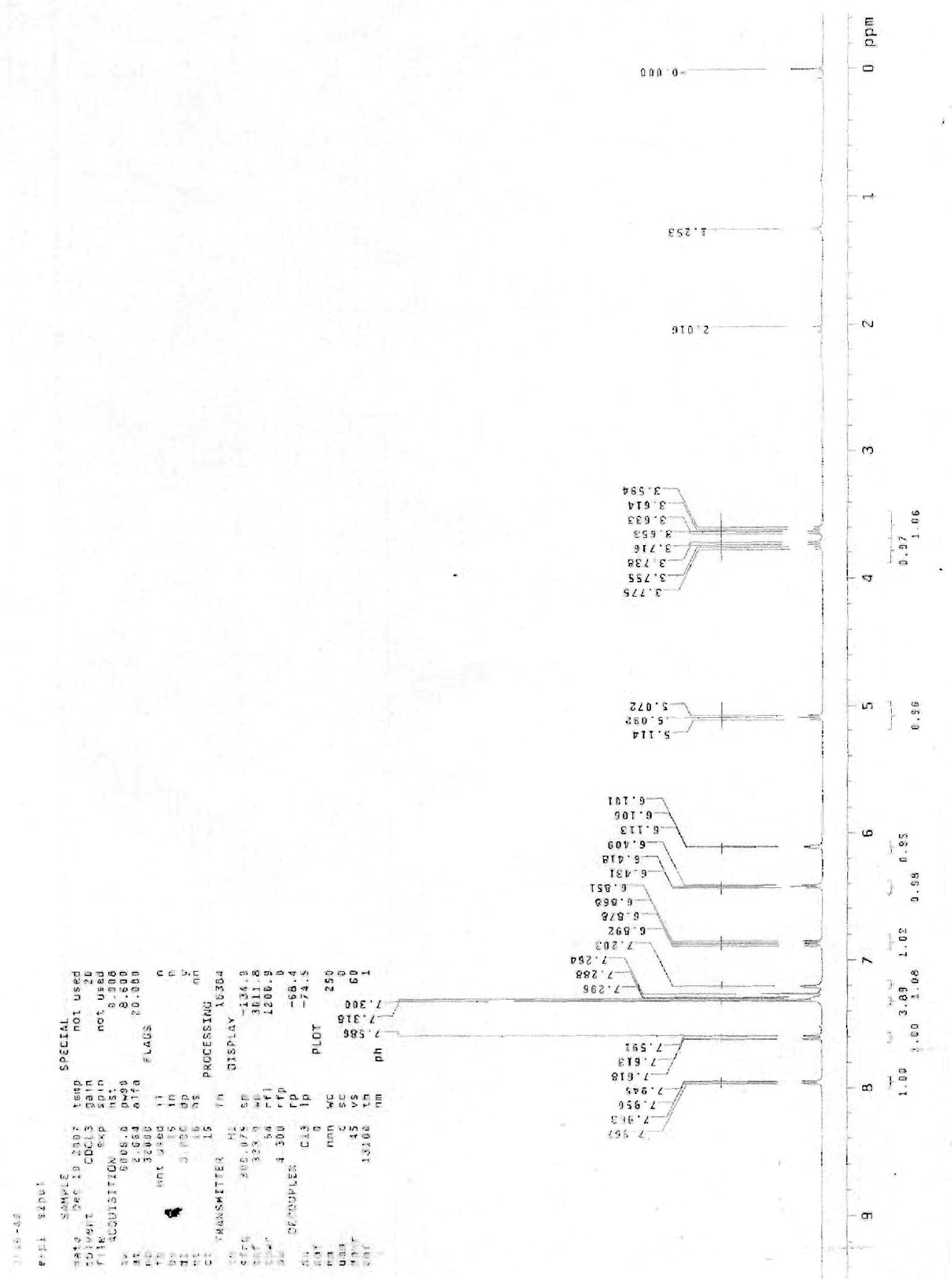

Fig. S-31: ${ }^{1} \mathrm{H}$ Spectra of 5,6-dihydro-6-hydroxymethyl-5-(4-chlorophenyl) pyrido[2,3-e $]$ pyrrolo[1,2-a]pyrazine $3 \mathrm{j}$. 


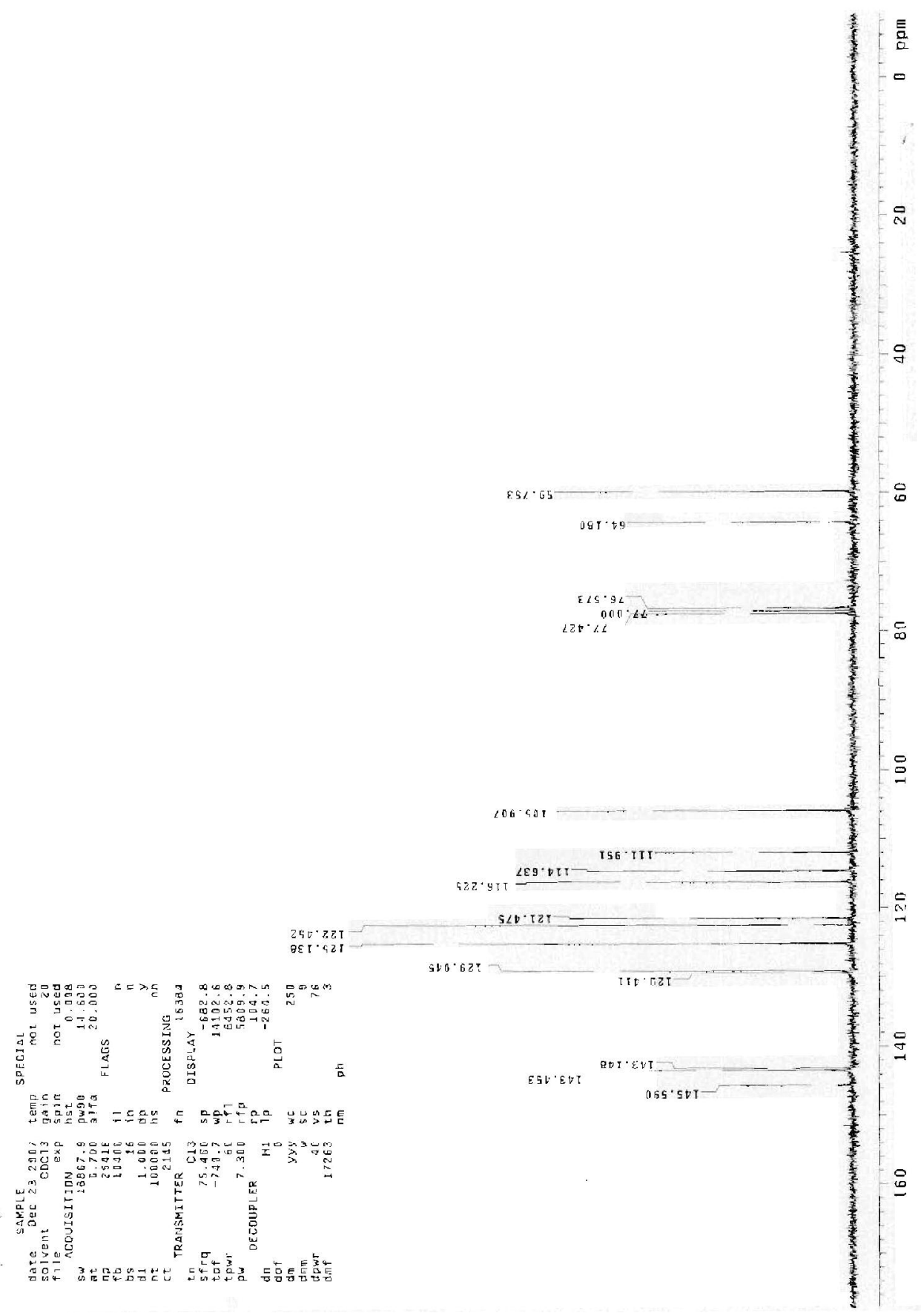

Fig. S-32: ${ }^{13} \mathrm{C}$ Spectra of 5,6-dihydro-6-hydroxymethyl-5-(4-chlorophenyl) pyrido[2,3-e]pyrrolo[1,2-a]pyrazine $3 \mathrm{j}$. 


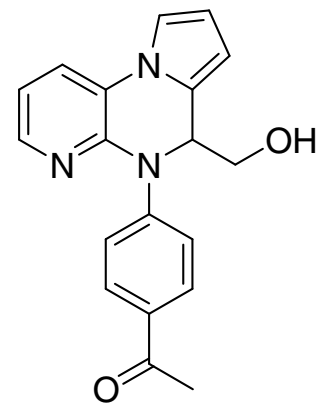

$\mathrm{C}_{19} \mathrm{H}_{17} \mathrm{~N}_{3} \mathrm{O}_{2}$

Exact Mass: 319.13

3k Mol. Wt.: 319.36
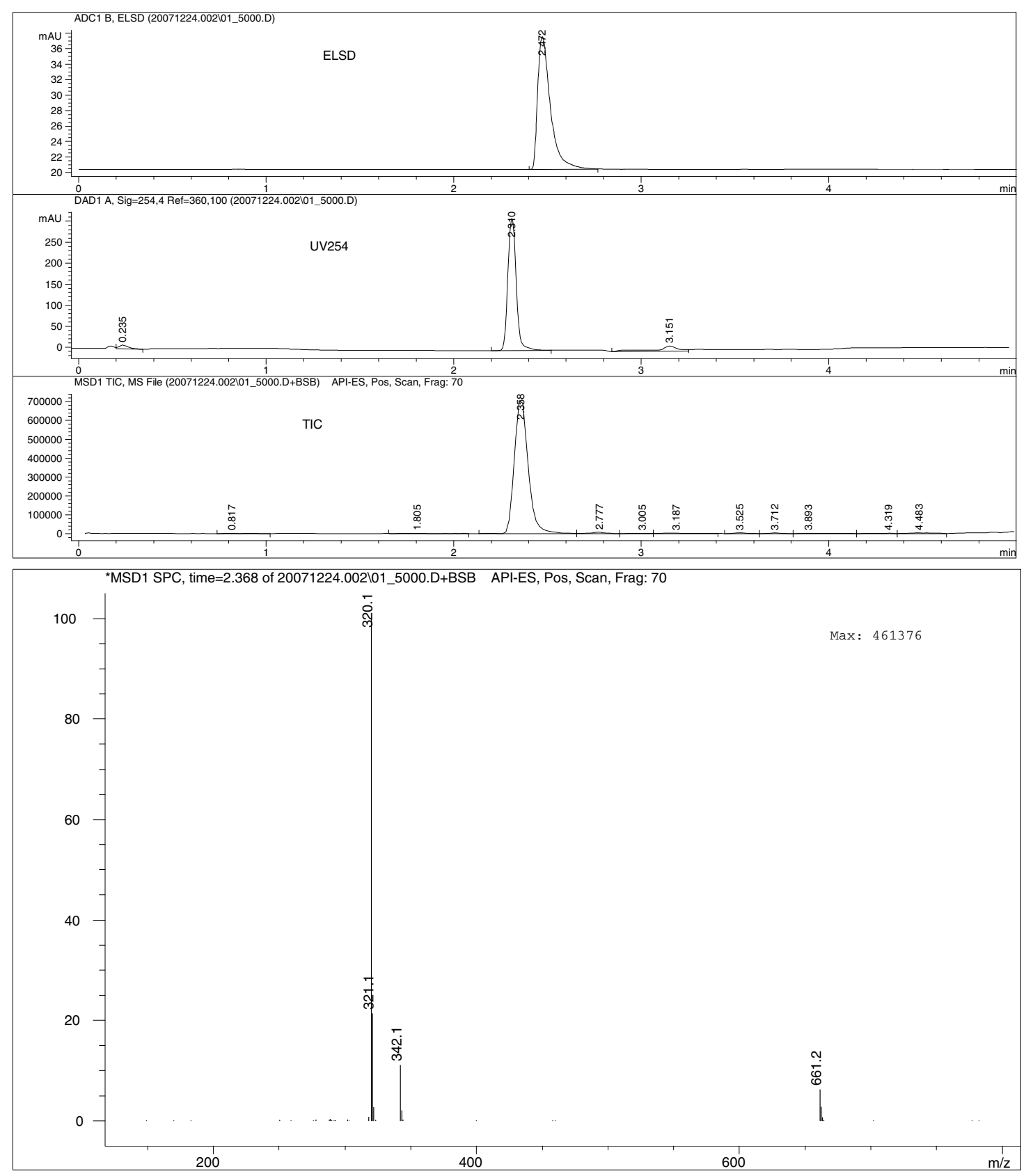

Fig. S-33: LC-MS-ELSD of 5,6-dihydro-6-hydroxymethyl-5-(4-acetylphenyl) pyrido[2,3-e $]$ pyrrolo[1,2-a]pyrazine 3k. 


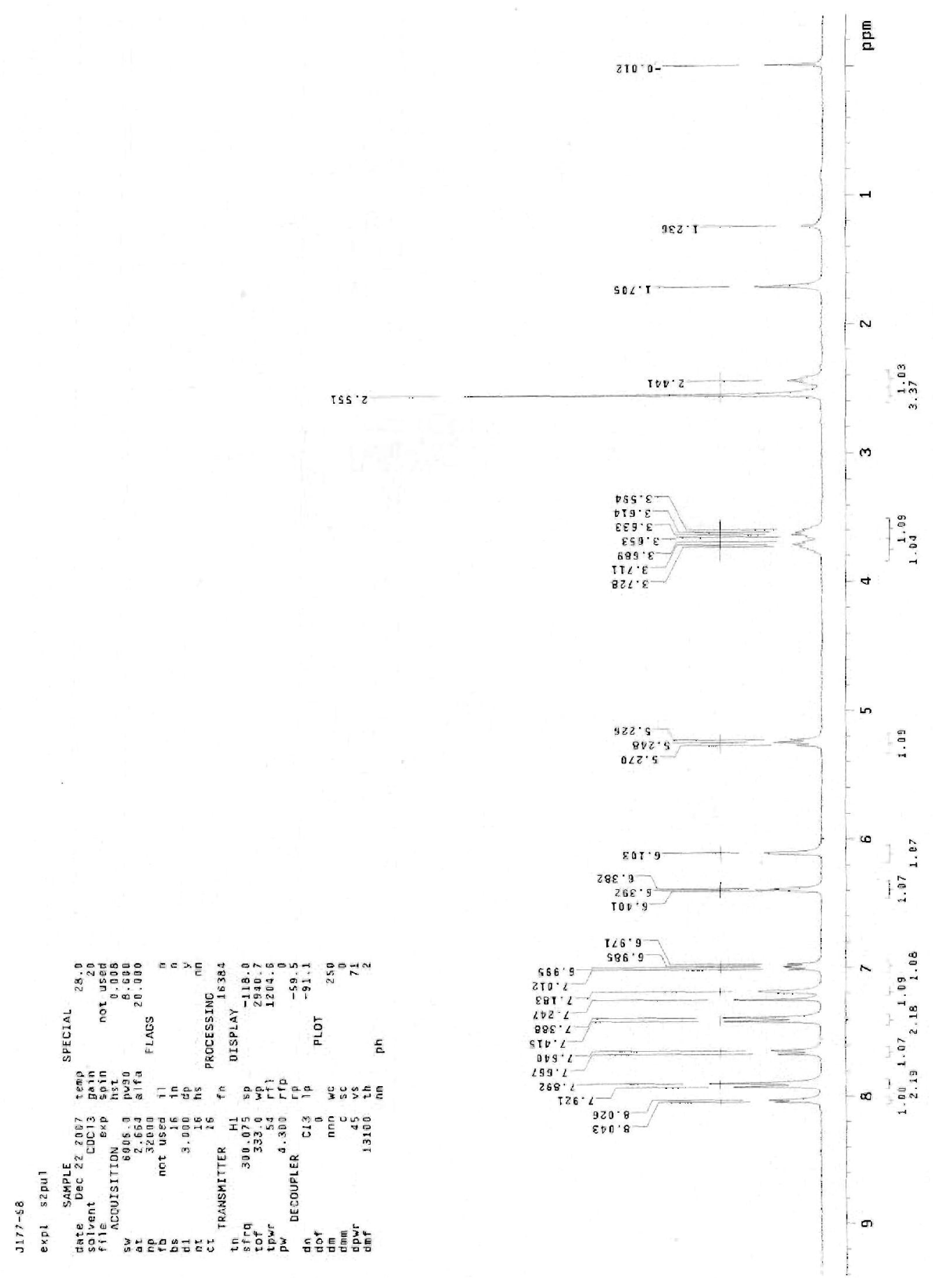

Fig. S-34: $\quad{ }^{1} \mathrm{H} \quad$ Spectra of 5,6-dihydro-6-hydroxymethyl-5-(4-acetylphenyl) pyrido[2,3-e]pyrrolo[1,2-a]pyrazine $3 \mathrm{k}$. 


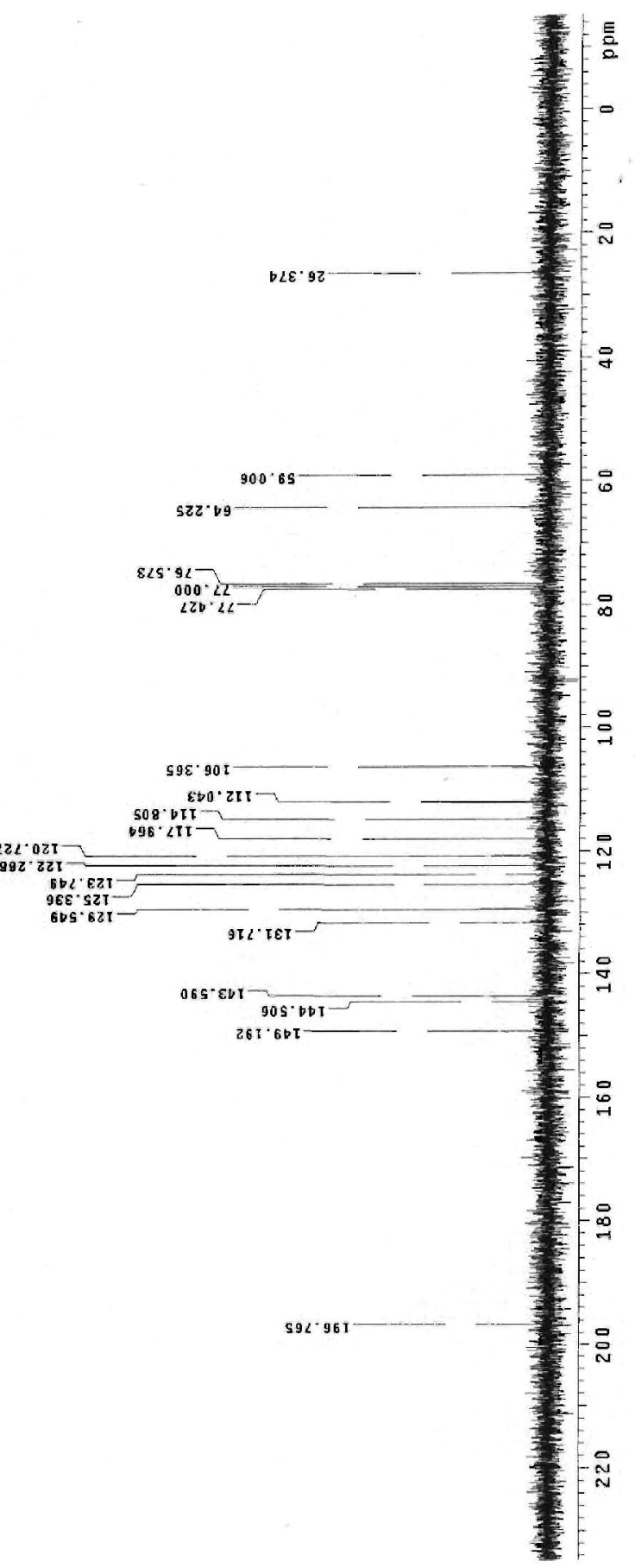

Fig. S-35: ${ }^{13} \mathrm{C}$ Spectra of 5,6-dihydro-6-hydroxymethyl-5-(4-acetylphenyl) pyrido[2,3-e]pyrrolo[1,2-a]pyrazine $3 \mathrm{k}$. 


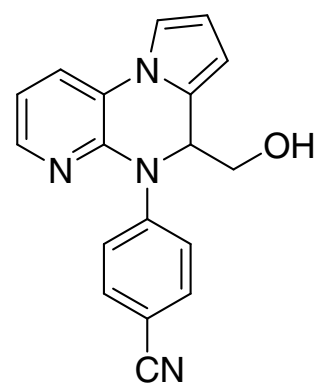

$\mathrm{C}_{18} \mathrm{H}_{14} \mathrm{~N}_{4} \mathrm{O}$

Exact Mass: 302.12

31 Mol. Wt.: 302.33
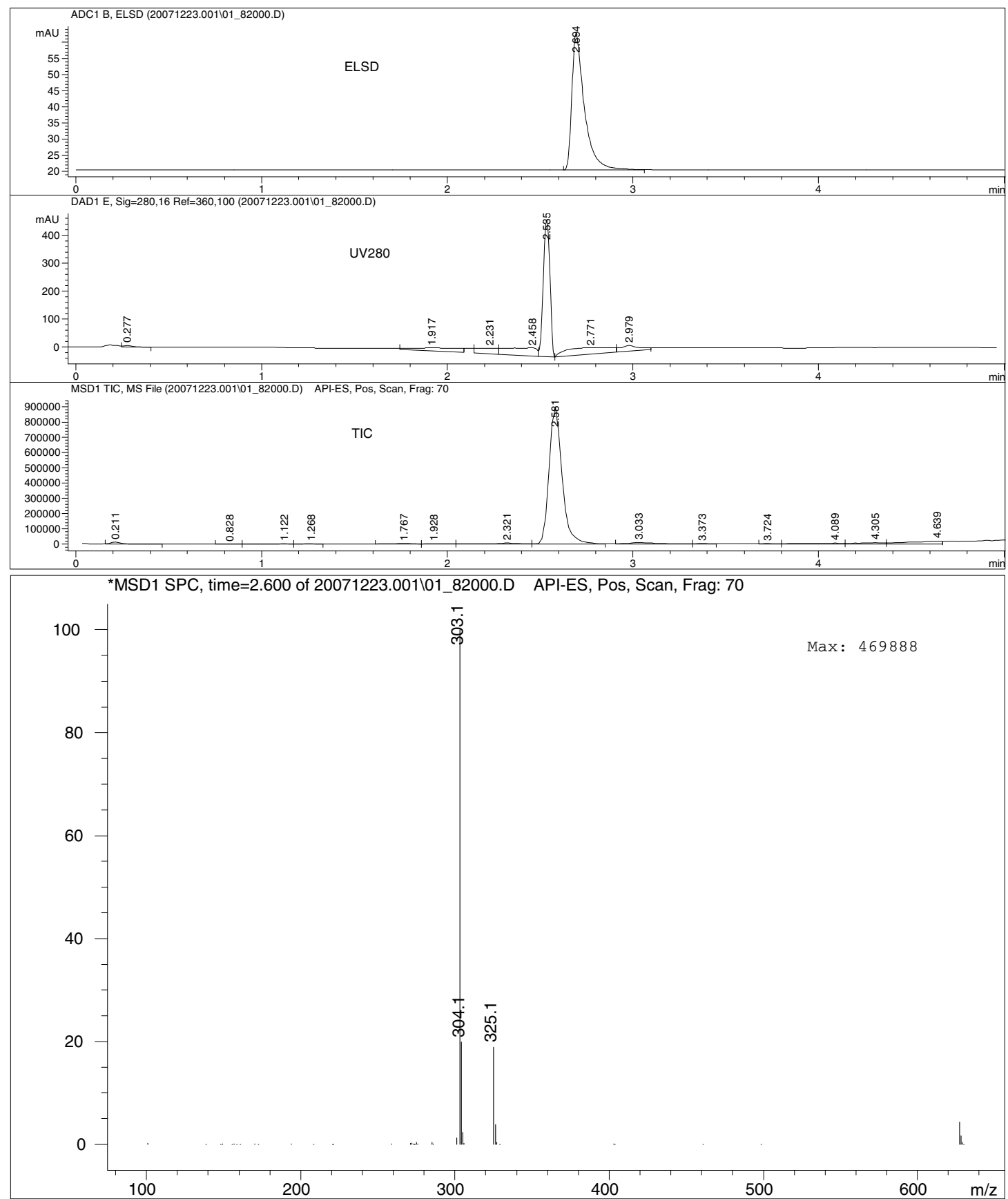

Fig. S-36: LC-MS-ELSD of 5,6-dihydro-6-hydroxymethyl-5-(4-cyanophenyl) pyrido[2,3-e]pyrrolo[1,2-a]pyrazine 31 . 


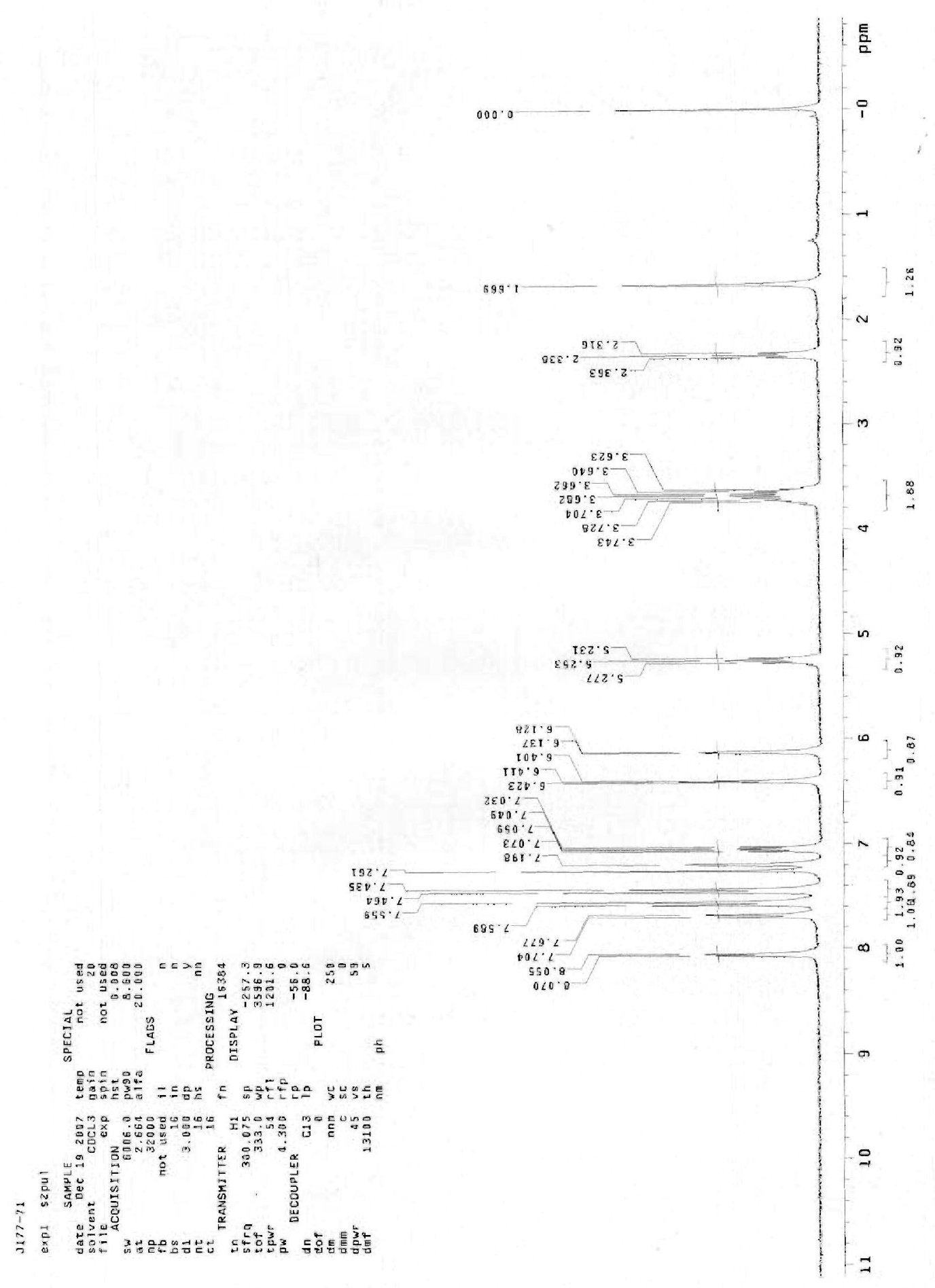

Fig. S-37: ${ }^{1} \mathrm{H} \quad$ Spectra of 5,6-dihydro-6-hydroxymethyl-5-(4-cyanophenyl) pyrido[2,3-e]pyrrolo[1,2-a]pyrazine 31 . 


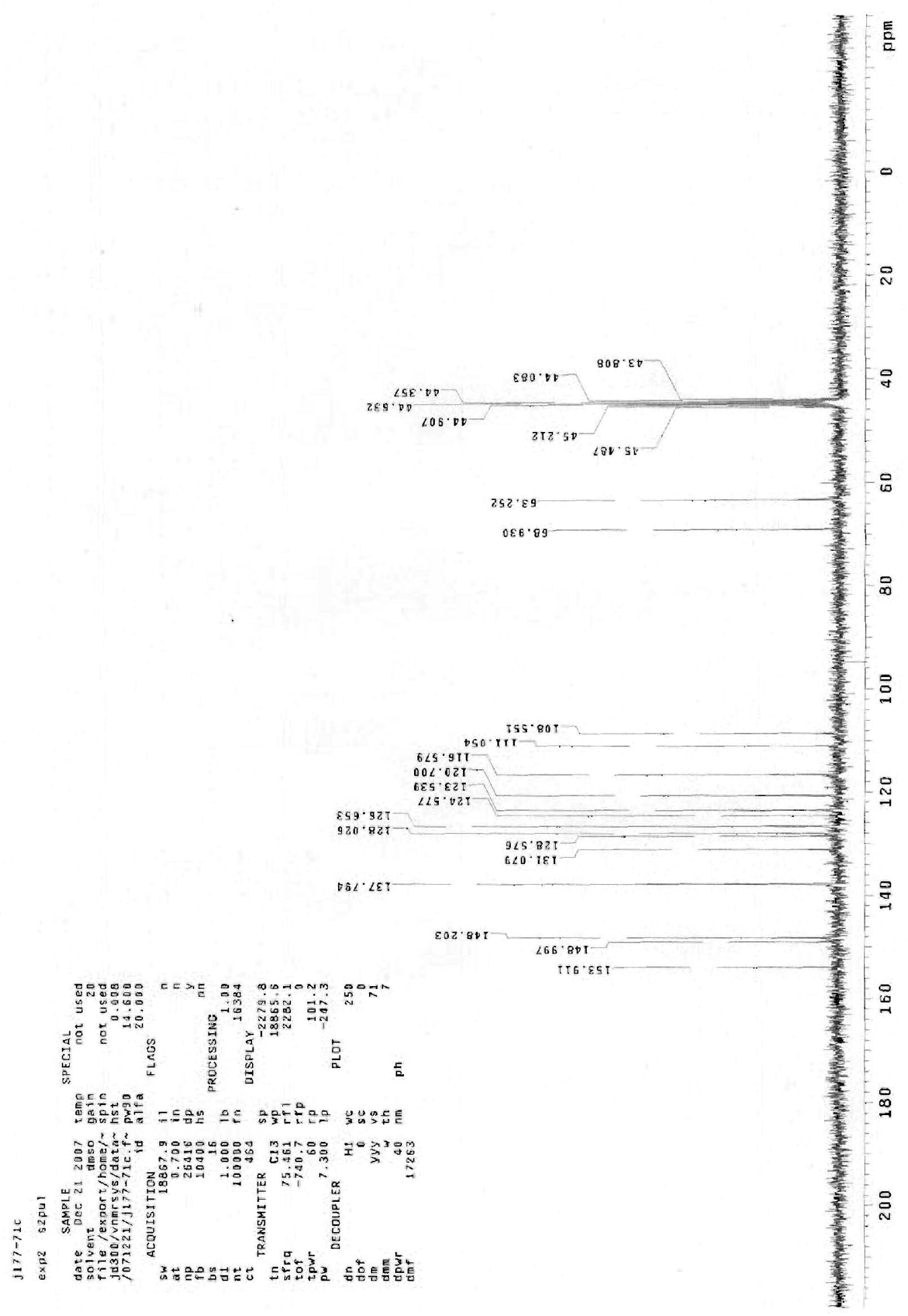

Fig. S-38: ${ }^{13} \mathrm{C}$ Spectra of 5,6-dihydro-6-hydroxymethyl-5-(4-cyanophenyl) pyrido[2,3-e $]$ pyrrolo[1,2-a]pyrazine 31 . 
<smiles>O=[N+]([O-])c1ccc(N2c3ncccc3-n3cccc3C2CO)cc1</smiles>

$\mathrm{C}_{17} \mathrm{H}_{14} \mathrm{~N}_{4} \mathrm{O}_{3}$

Exact Mass: 322.11

3m Mol. Wt.: 322.32

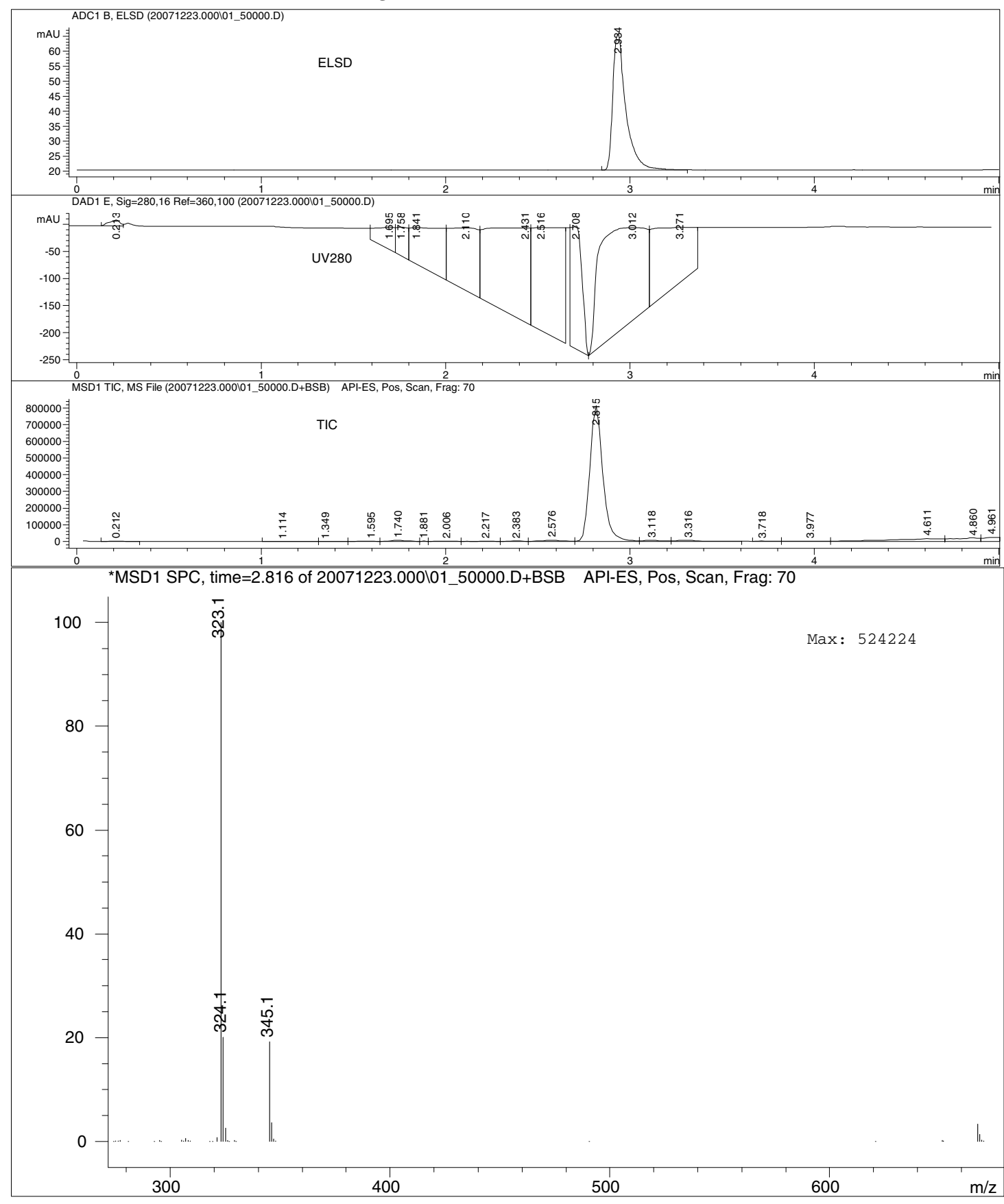

Fig. S-39: LC-MS-ELSD of 5,6-dihydro-6-hydroxymethyl-5-(4-nitrophenyl) pyrido[2,3-e $]$ pyrrolo[1,2-a]pyrazine $3 \mathrm{~m}$. 


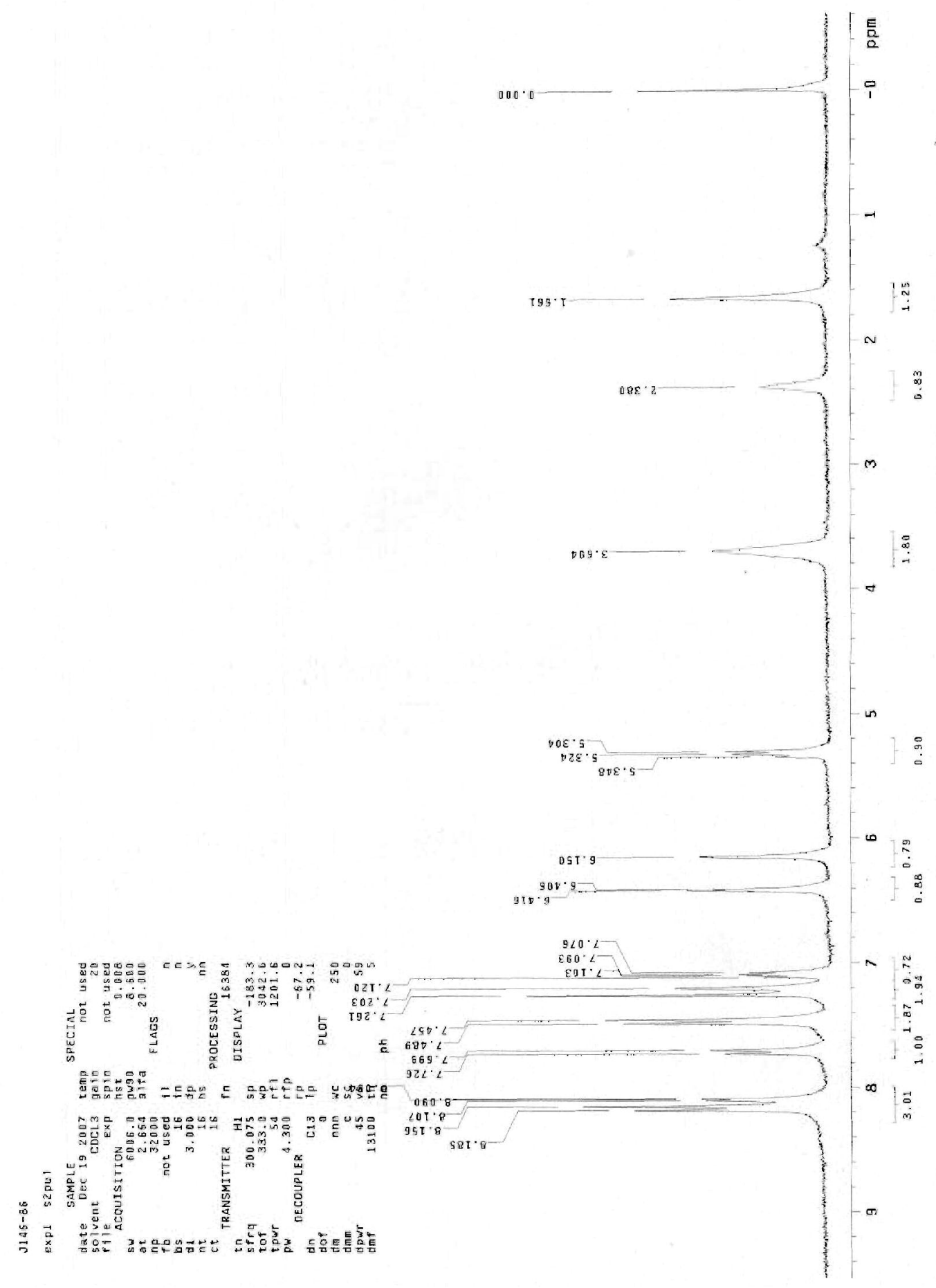

Fig. S-40: ${ }^{1} \mathrm{H}$ Spectra of 5,6-dihydro-6-hydroxymethyl-5-(4-nitrophenyl) pyrido[2,3-e $]$ pyrrolo[1,2-a]pyrazine $3 \mathrm{~m}$. 


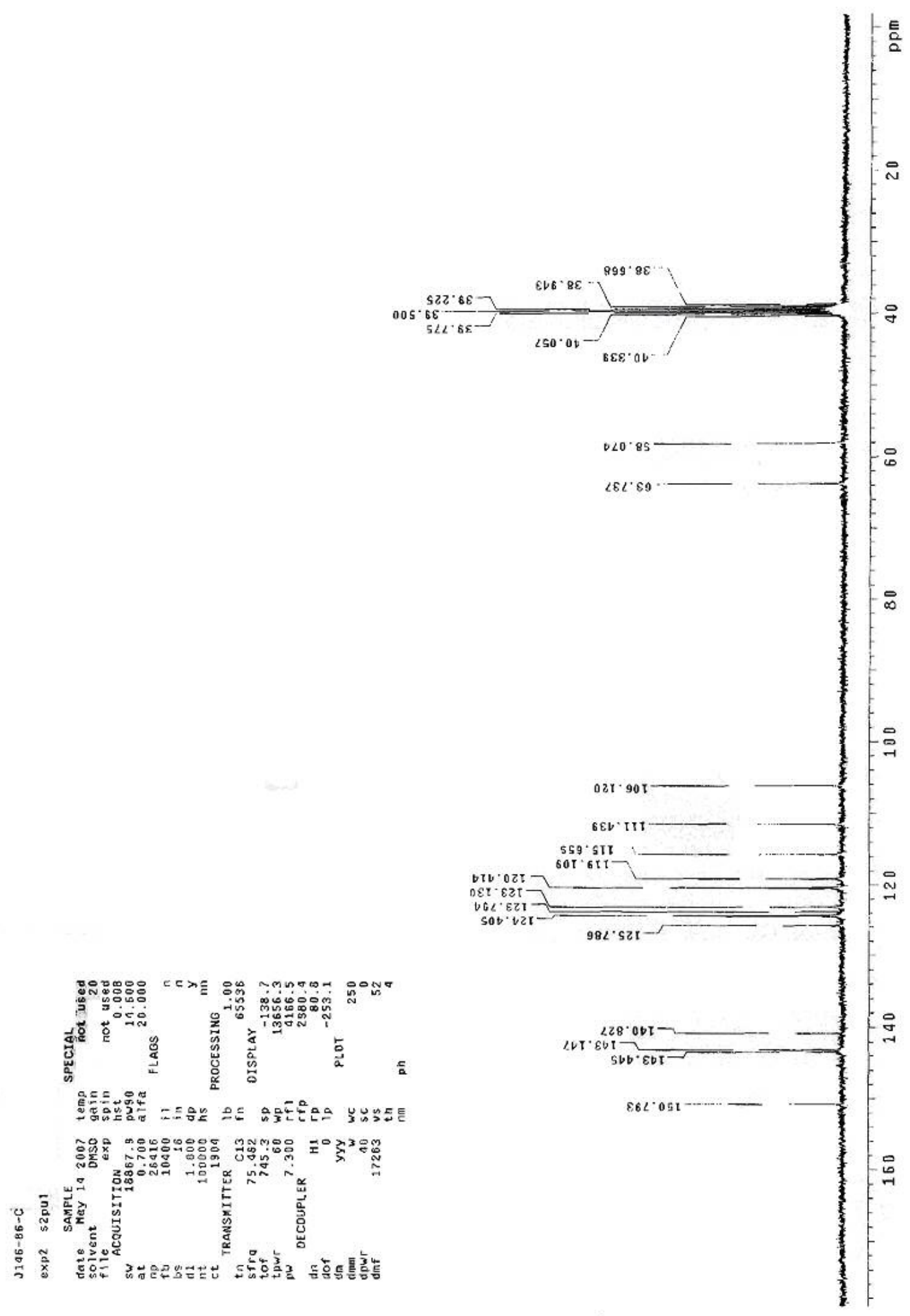

Fig. S-41: ${ }^{13} \mathrm{C}$ Spectra of 5,6-dihydro-6-hydroxymethyl-5-(4-nitrophenyl) pyrido[2,3-e $]$ pyrrolo[1,2-a]pyrazine $3 \mathrm{~m}$. 


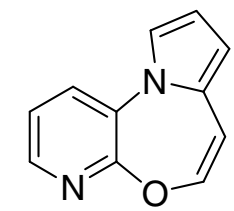

$\mathrm{C}_{11} \mathrm{H}_{8} \mathrm{~N}_{2} \mathrm{O}$

Exact Mass: 184.06

7 Mol. Wt.: 184.19

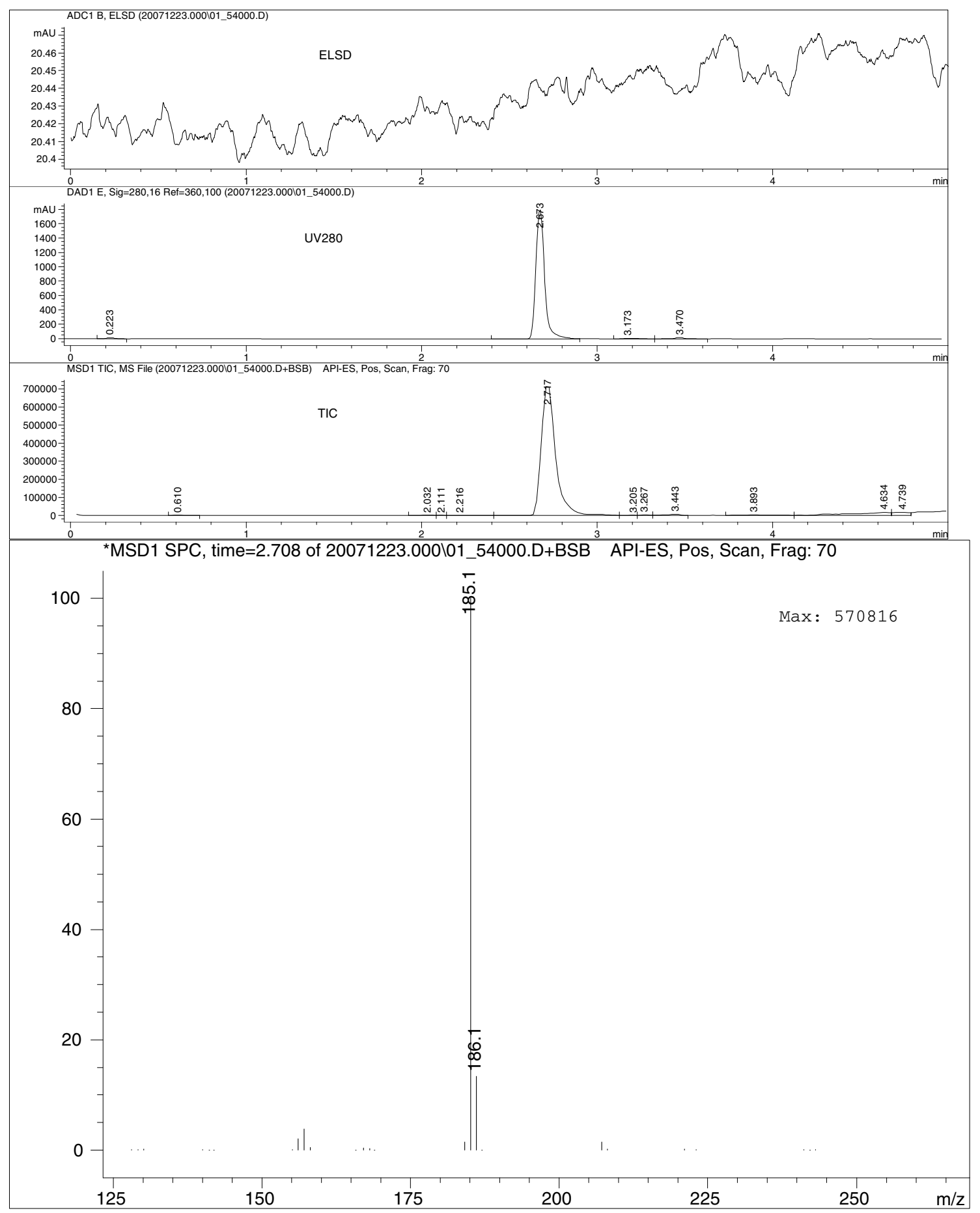

Fig. S-42: LC-MS-ELSD of pyrido[2,3-b]pyrrolo[1,2-d][1,4]oxazepine 7. 

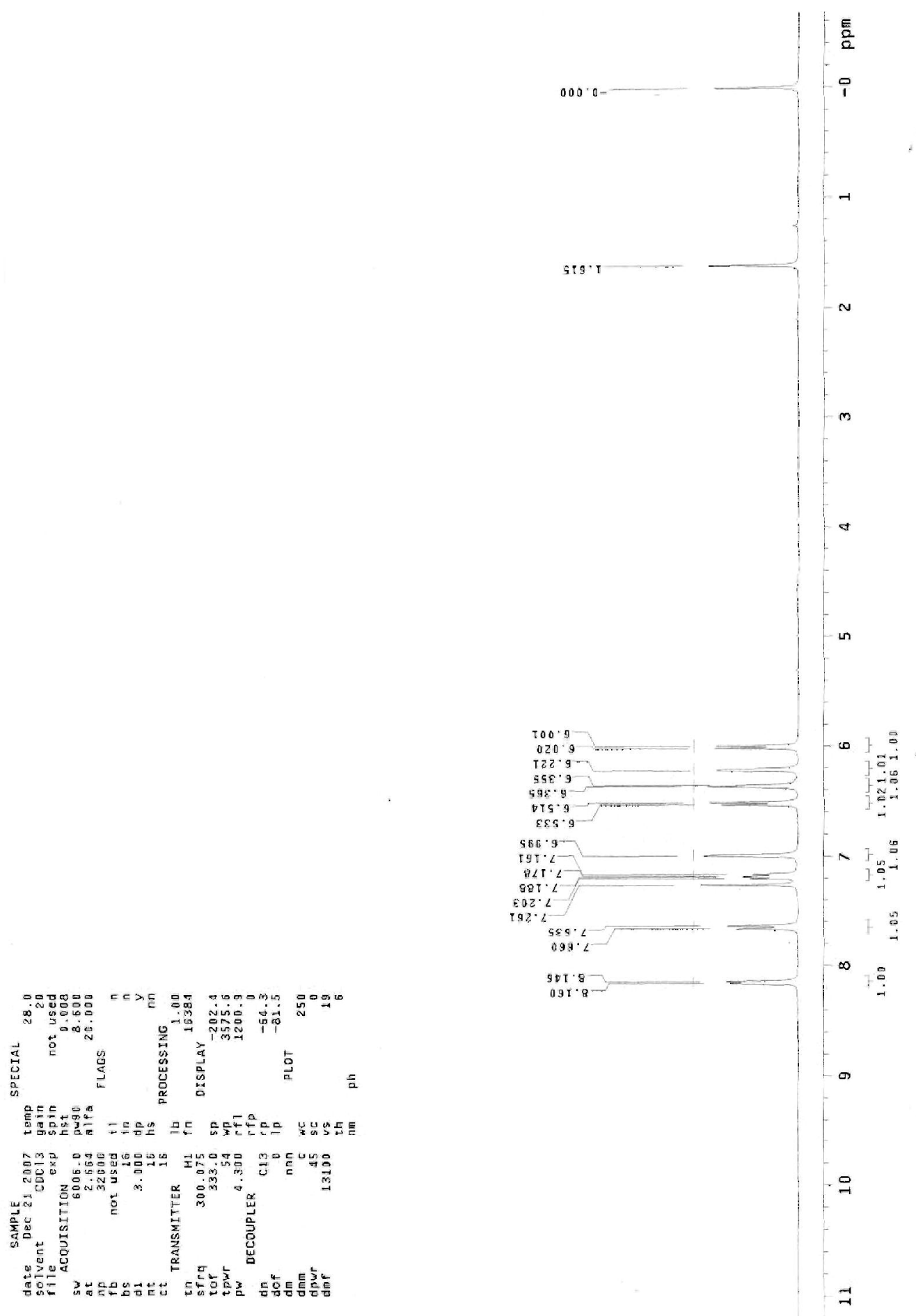

Fig. S-43: ${ }^{1} \mathrm{H}$ Spectra of pyrido[2,3-b]pyrrolo[1,2-d][1,4]oxazepine 7. 


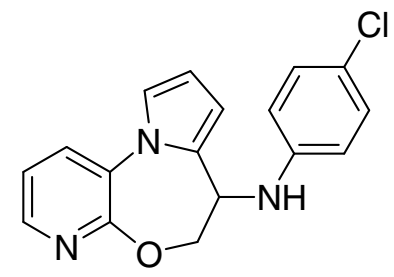

$\mathrm{C}_{17} \mathrm{H}_{14} \mathrm{ClN}_{3} \mathrm{O}$

Exact Mass: 311.08

2j Mol. Wt.: 311.77

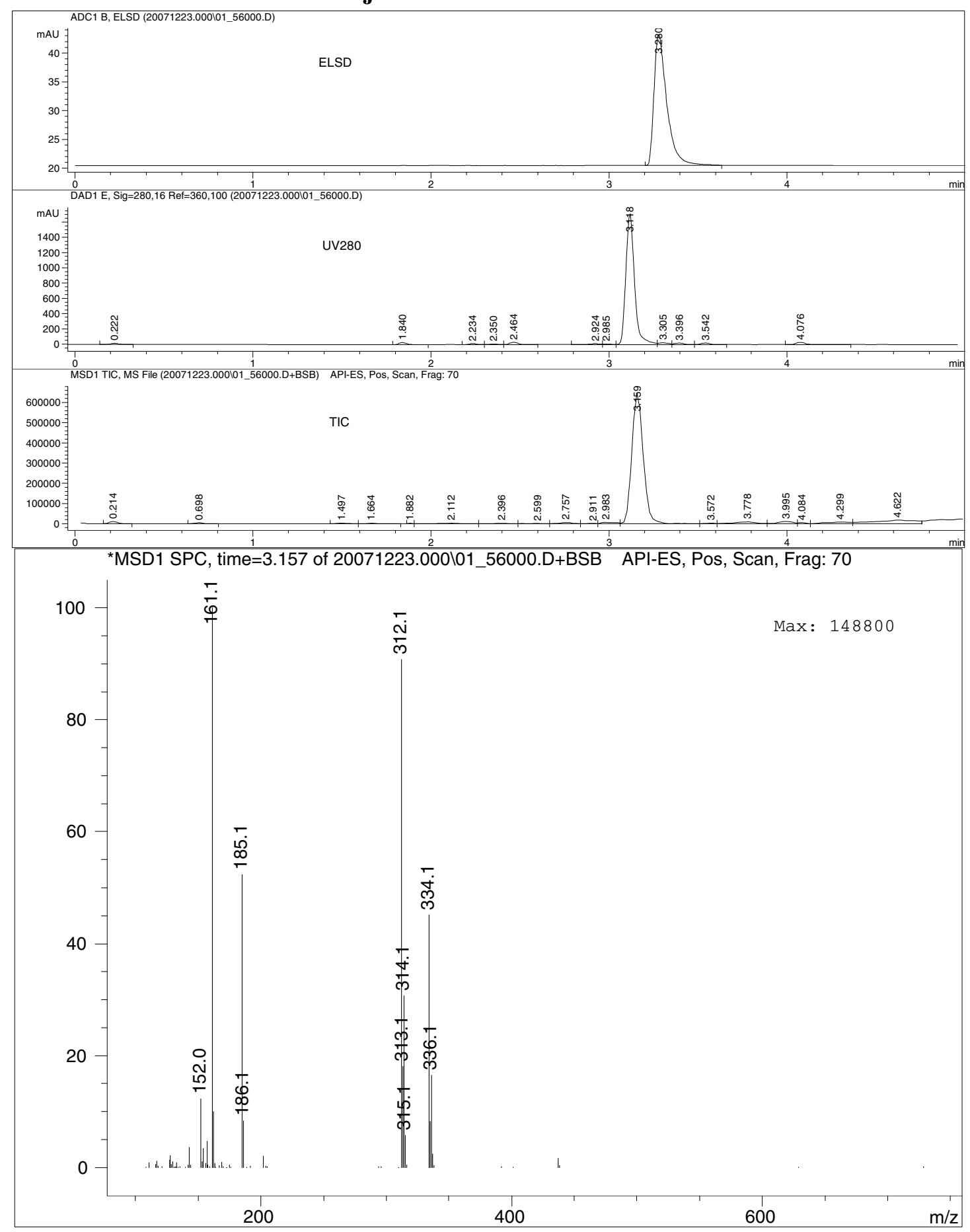

Fig. S-44: LC-MS-ELSD of 4-chlorophenylamino-6,7-dihydropyrido[2,3-b] pyrrolo[1,2-d][1,4]oxazepine $2 \mathrm{j}$. 


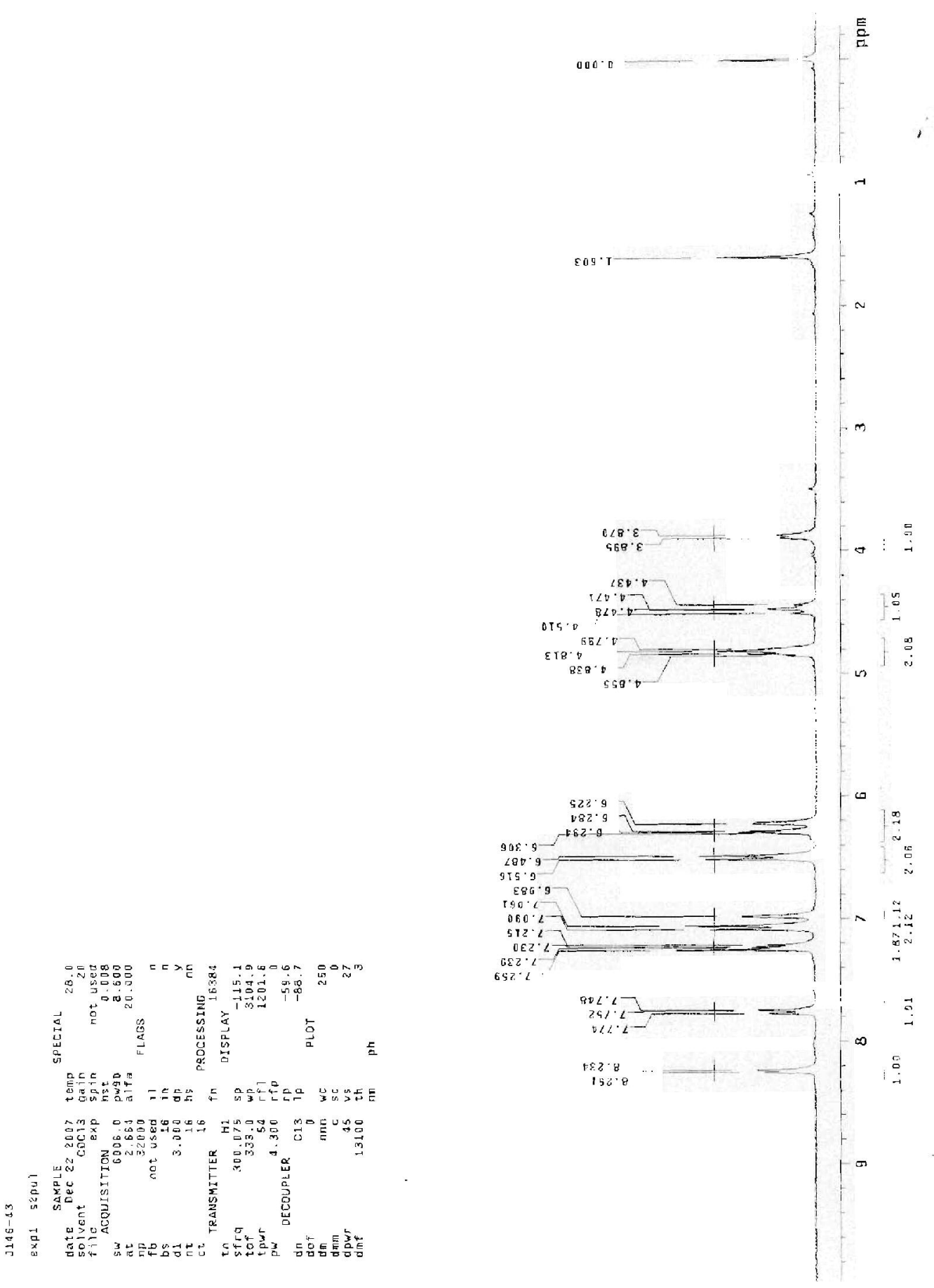

Fig. S-45: ${ }^{1} \mathrm{H}$ Spectra of 4-chlorophenylamino-6,7-dihydropyrido[2,3-b] pyrrolo[1,2-d][1,4]oxazepine $2 \mathrm{j}$. 


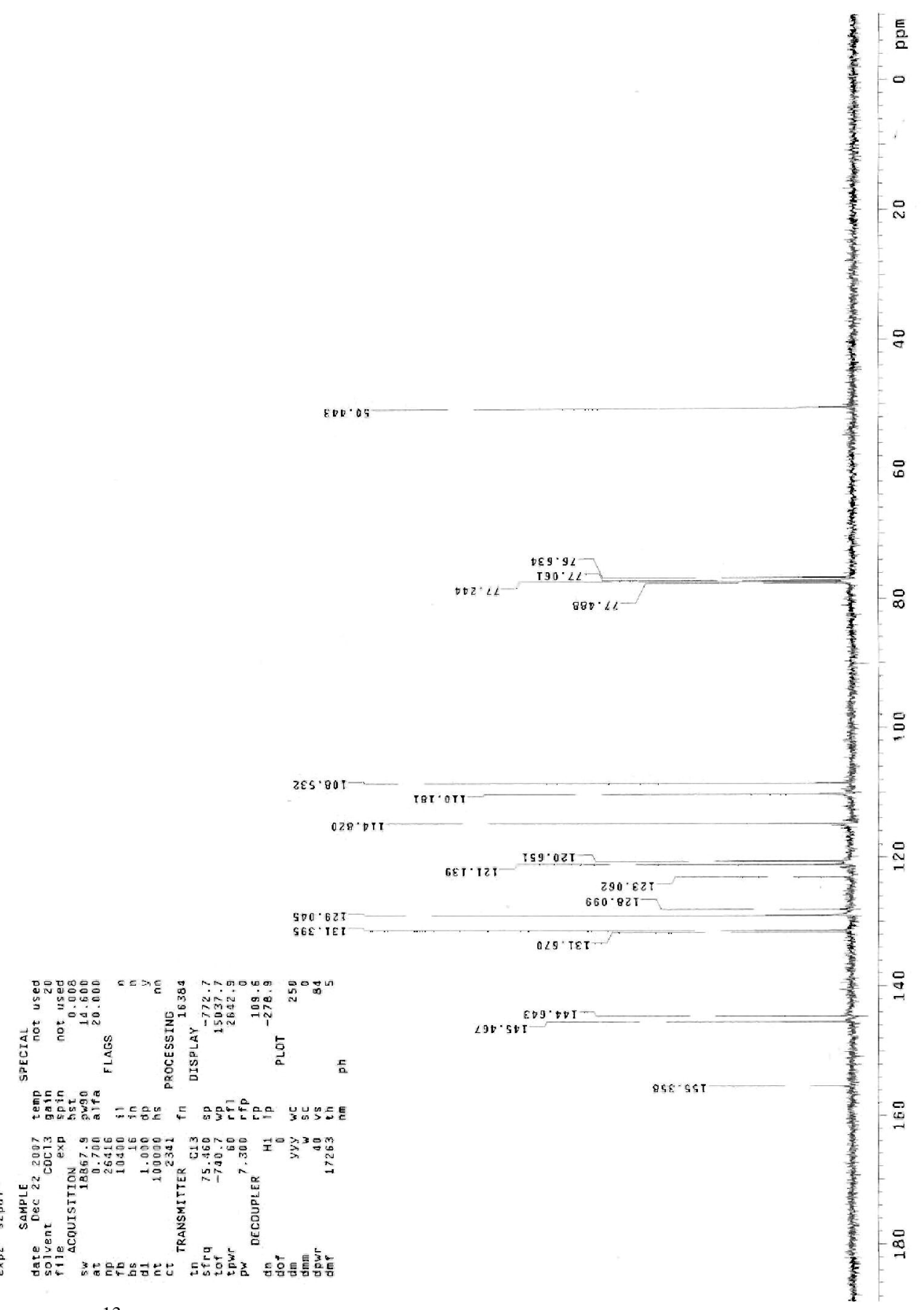

Fig. S-46: ${ }^{13} \mathrm{C}$ Spectra of 4-chlorophenylamino-6,7-dihydropyrido[2,3-b] pyrrolo[1,2-d][1,4]oxazepine $2 \mathrm{j}$. 


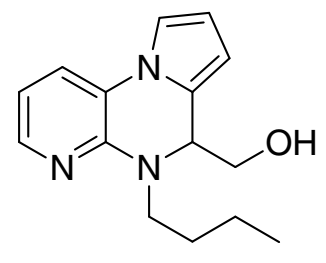

$\mathrm{C}_{15} \mathrm{H}_{19} \mathrm{~N}_{3} \mathrm{O}$

Exact Mass: 257.15

3n Mol. Wt.: 257.33
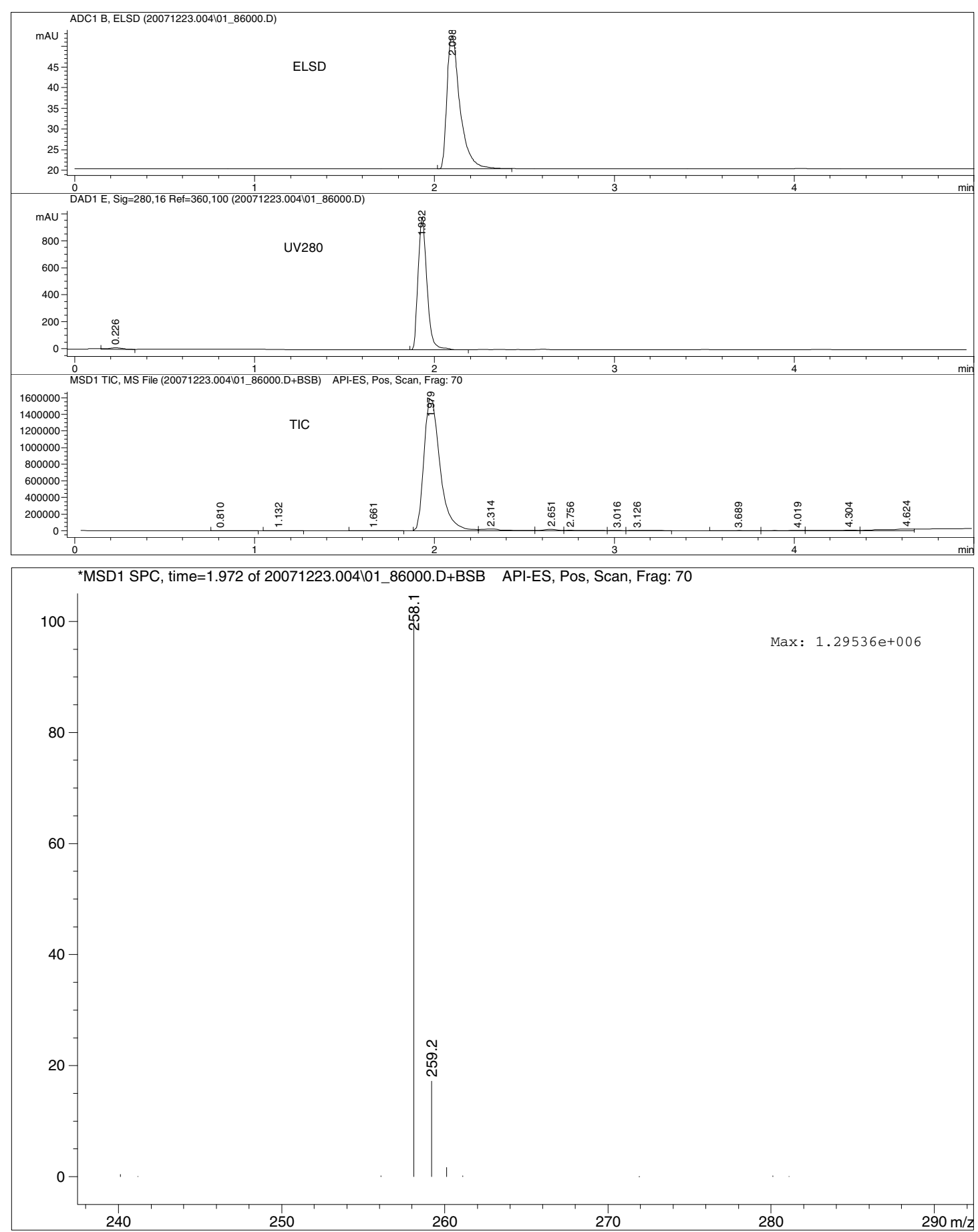

Fig. S-47: LC-MS-ELSD of 5-(n-butyl)-5,6-dihydro-6-hydroxymethylpyrido [2,3-e]pyrrolo[1,2-a]pyrazine 3n. 

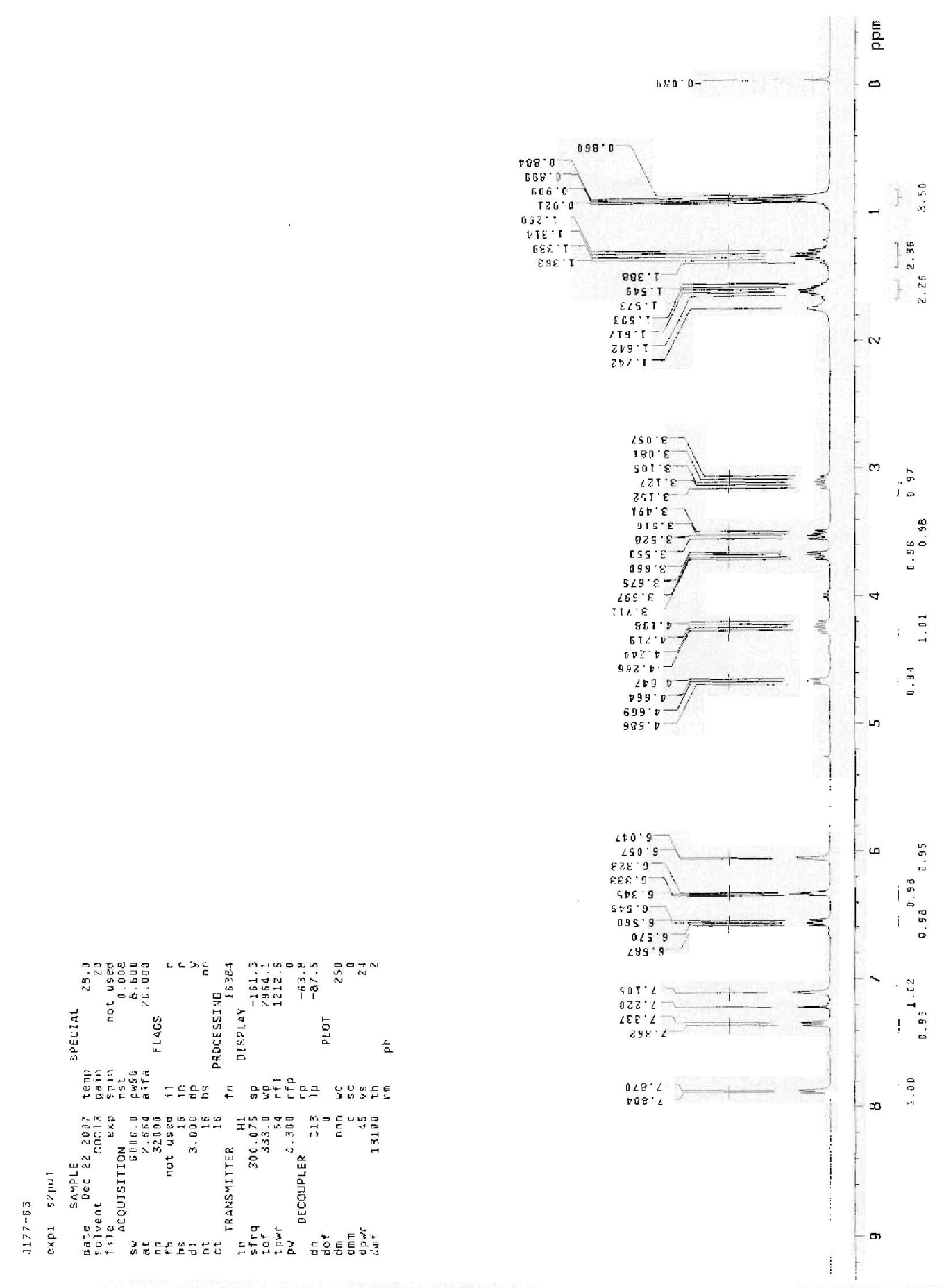

Fig. S-48: ${ }^{1} \mathrm{H} \quad$ Spectra of 5-(n-butyl)-5,6-dihydro-6-hydroxymethylpyrido [2,3-e]pyrrolo[1,2-a]pyrazine 3n. 


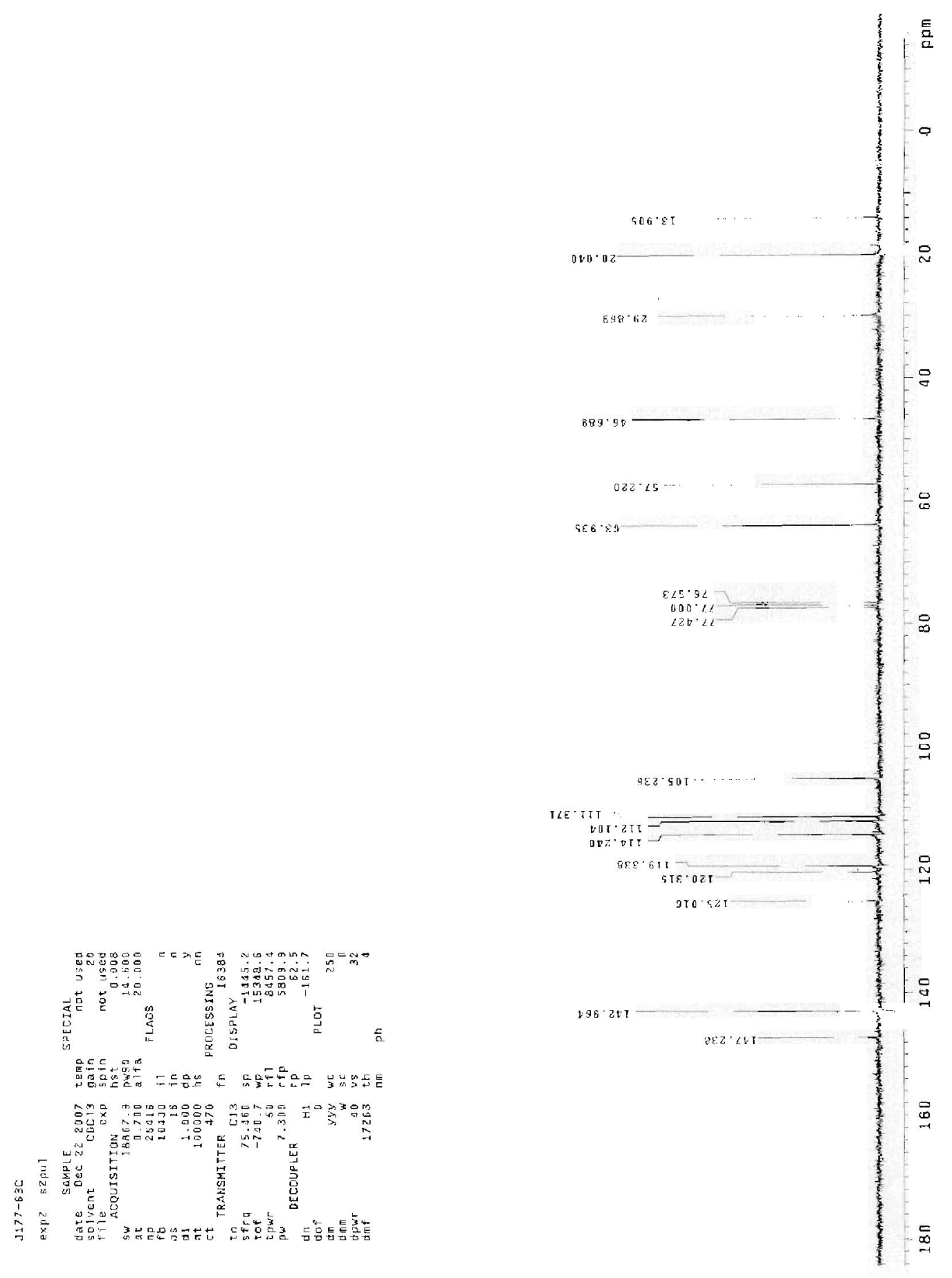

Fig. S-49: ${ }^{13} \mathrm{C}$ Spectra of 5-(n-butyl)-5,6-dihydro-6-hydroxymethylpyrido [2,3-e]pyrrolo[1,2-a]pyrazine 3n. 


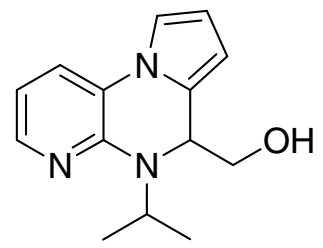

$\mathrm{C}_{14} \mathrm{H}_{17} \mathrm{~N}_{3} \mathrm{O}$

Exact Mass: 243.14

3o Mol. Wt.: 243.3

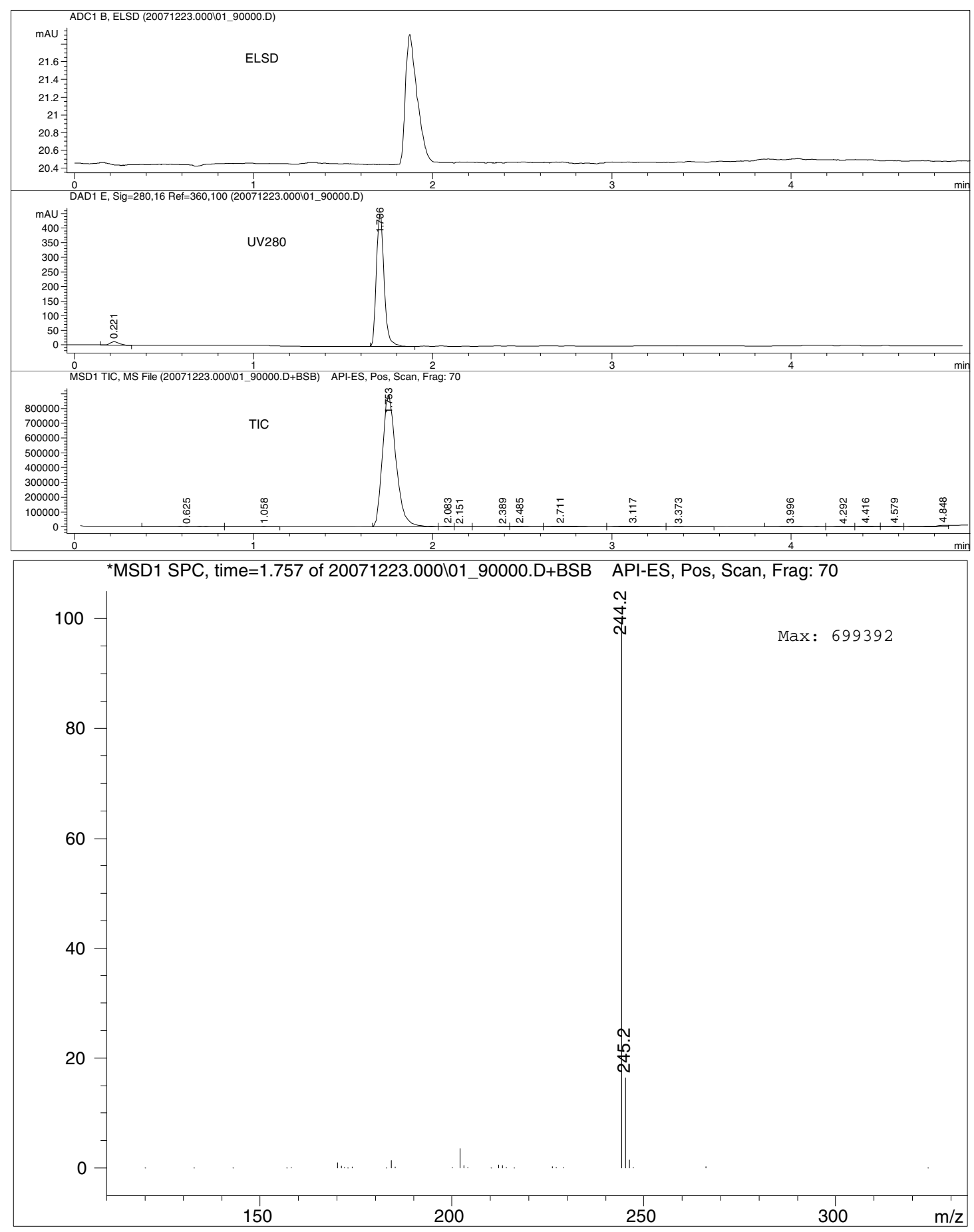

Fig. S-50: LC-MS-ELSD of 5,6-dihydro-6-hydroxymethyl-5-(iso-propyl)pyrido [2,3-e]pyrrolo[1,2-a]pyrazine 30. 


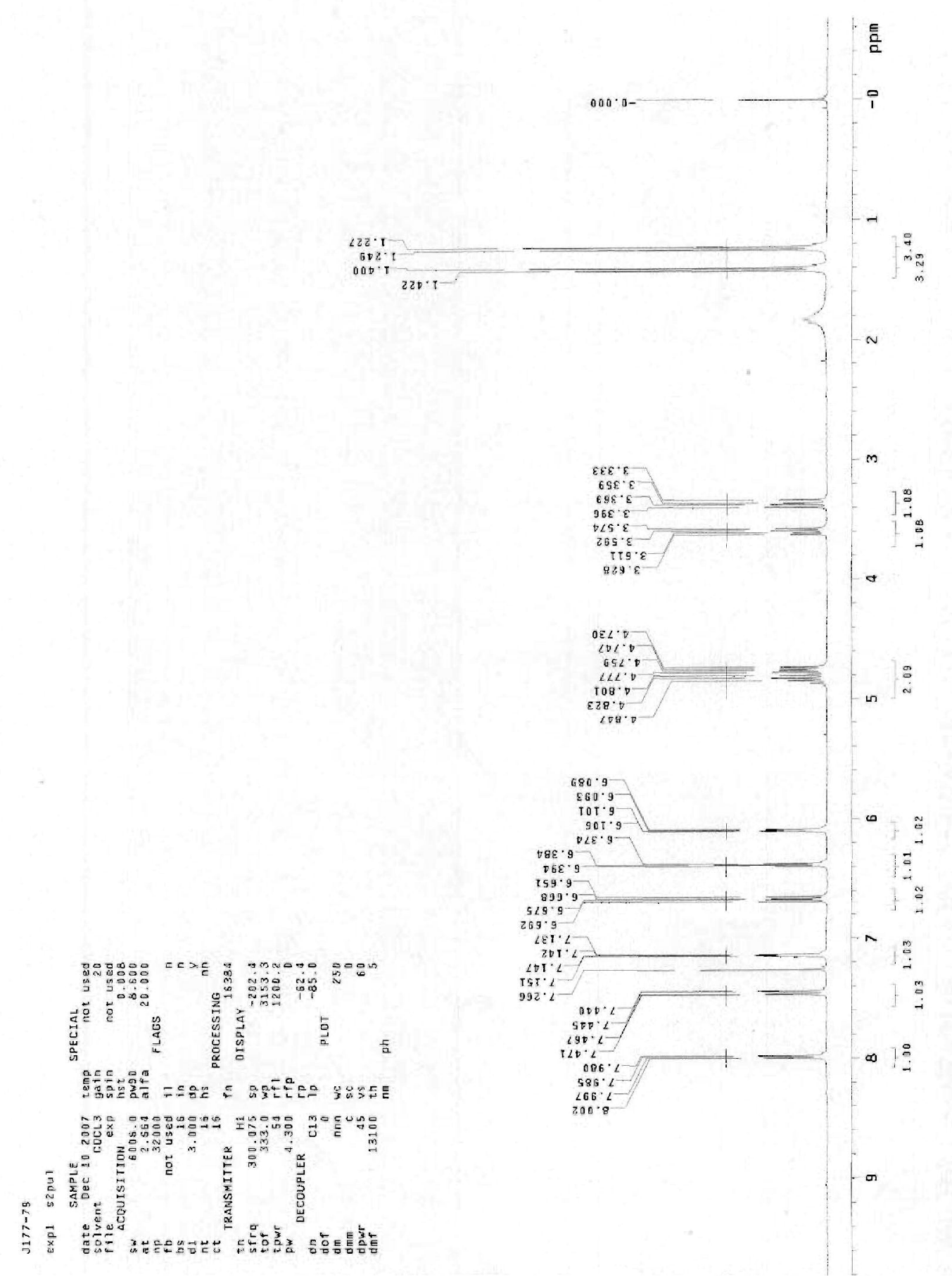

Fig. S-51: ${ }^{1} \mathrm{H}$ Spectra of 5,6-dihydro-6-hydroxymethyl-5-(iso-propyl)pyrido [2,3-e]pyrrolo[1,2-a]pyrazine 30 . 


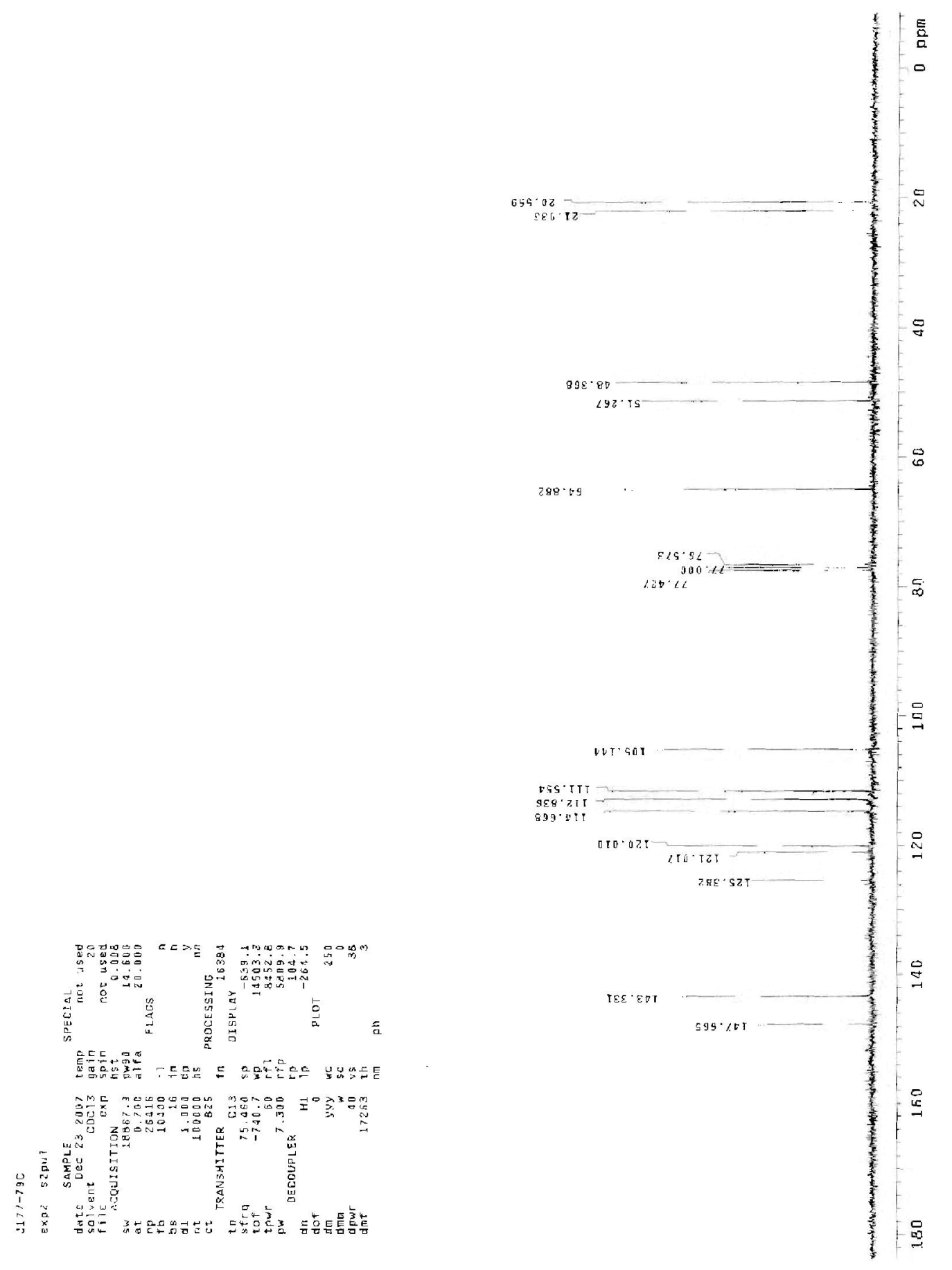

Fig. S-52: ${ }^{13} \mathrm{C}$ Spectra of 5,6-dihydro-6-hydroxymethyl-5-(iso-propyl)pyrido [2,3-e]pyrrolo[1,2-a]pyrazine 30 . 


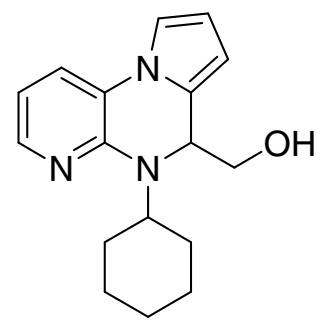

$\mathrm{C}_{17} \mathrm{H}_{21} \mathrm{~N}_{3} \mathrm{O}$

Exact Mass: 283.17

3p Mol. Wt.: 283.37

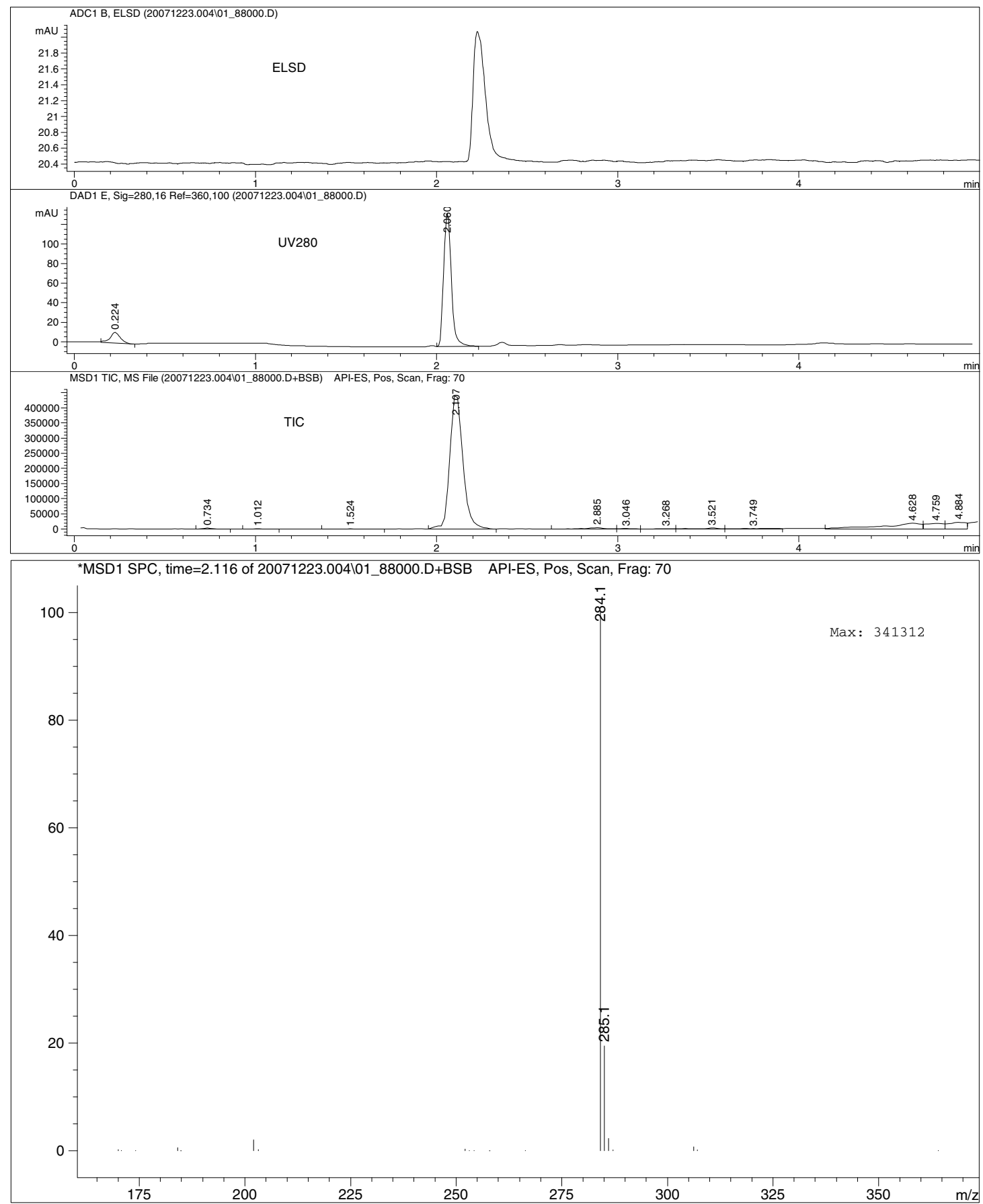

Fig. S-53: LC-MS-ELSD of 5-cyclohexyl-5,6-dihydro-6-hydroxymethylpyrido [2,3-e]pyrrolo[1,2-a]pyrazine 3p. 


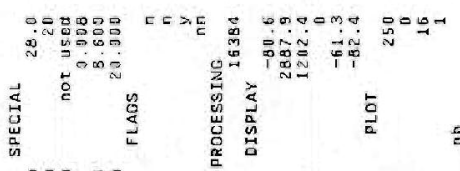

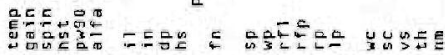

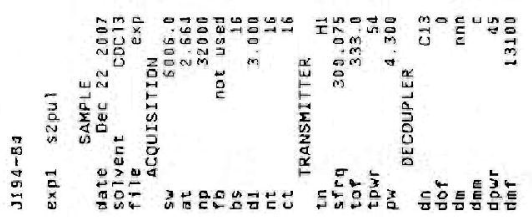

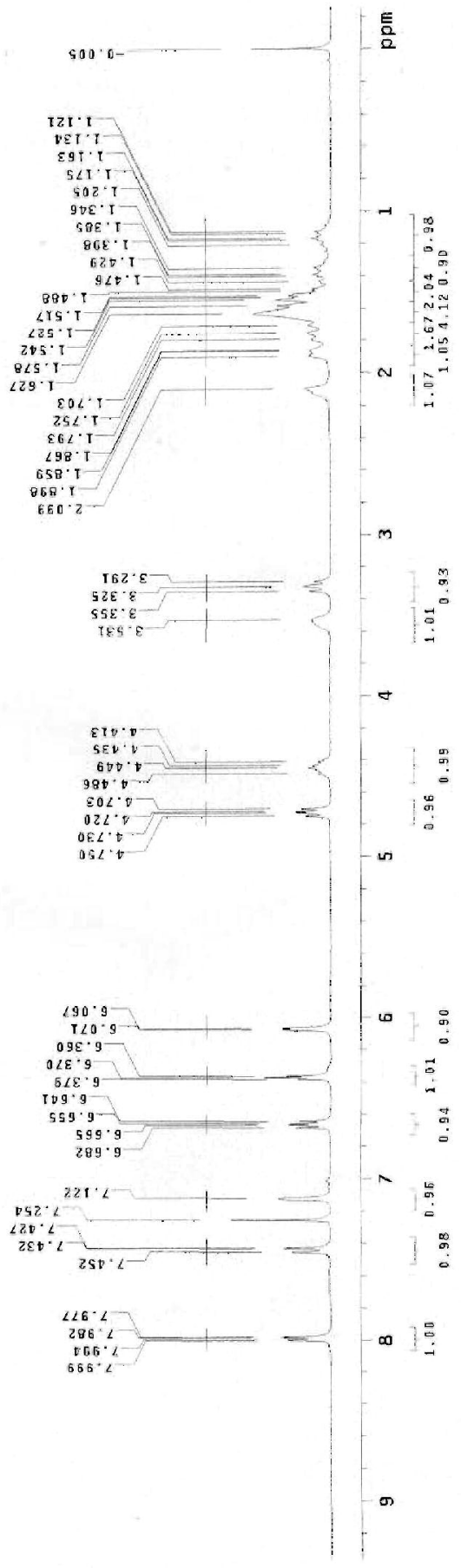

Fig. S-54: ${ }^{1} \mathrm{H}$ Spectra of 5-cyclohexyl-5,6-dihydro-6-hydroxymethylpyrido $[2,3-e]$ pyrrolo $[1,2-a]$ pyrazine $3 p$. 

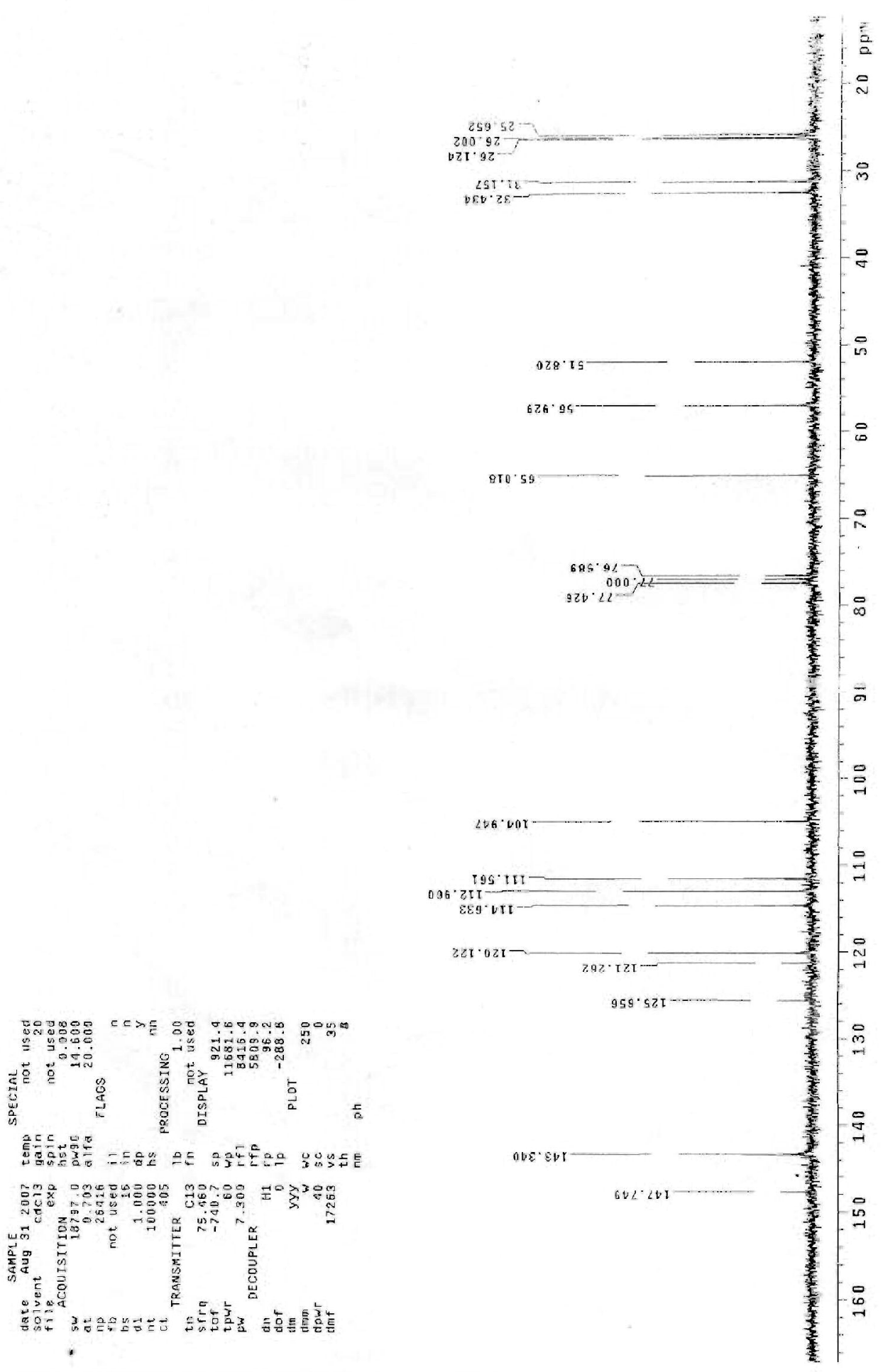

Fig. S-55: ${ }^{13} \mathrm{C}$ Spectra of 5-cyclohexyl-5,6-dihydro-6-hydroxymethylpyrido [2,3-e]pyrrolo[1,2-a]pyrazine 3p. 


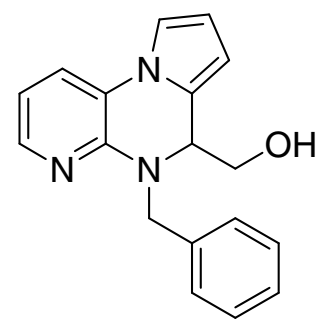

$\mathrm{C}_{18} \mathrm{H}_{17} \mathrm{~N}_{3} \mathrm{O}$

Exact Mass: 291.14

3q Mol. Wt.: 291.35

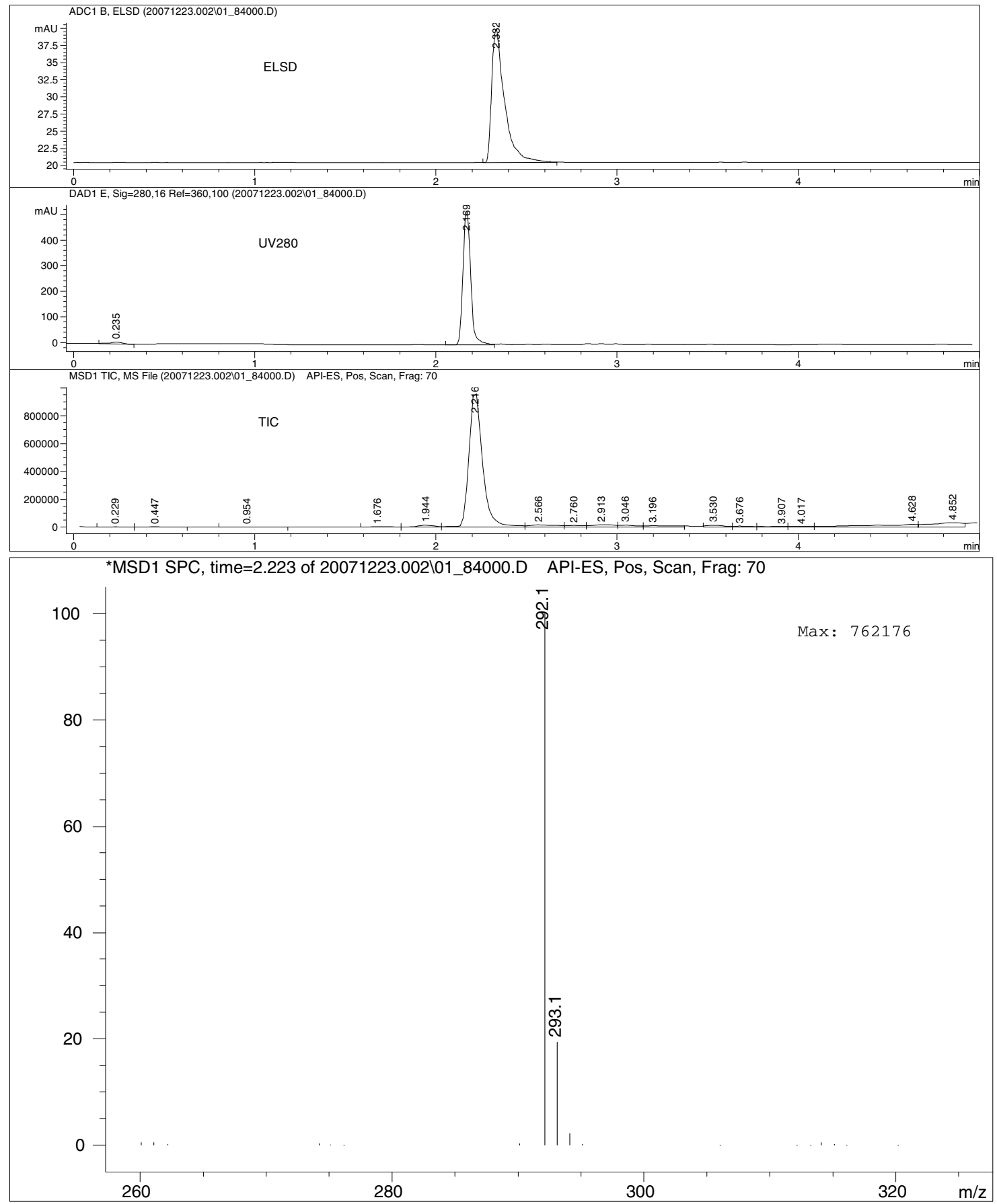

Fig. S-56: LC-MS-ELSD of 5-benzyl-5,6-dihydro-6-hydroxymethylpyrido [2,3-e $]$ pyrrolo[1,2-a]pyrazine 3q. 


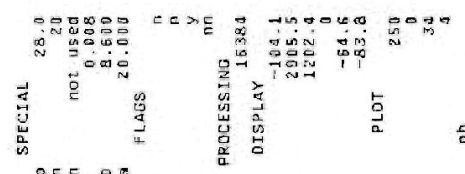

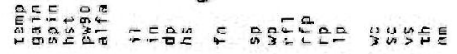

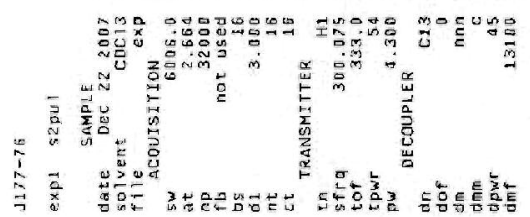

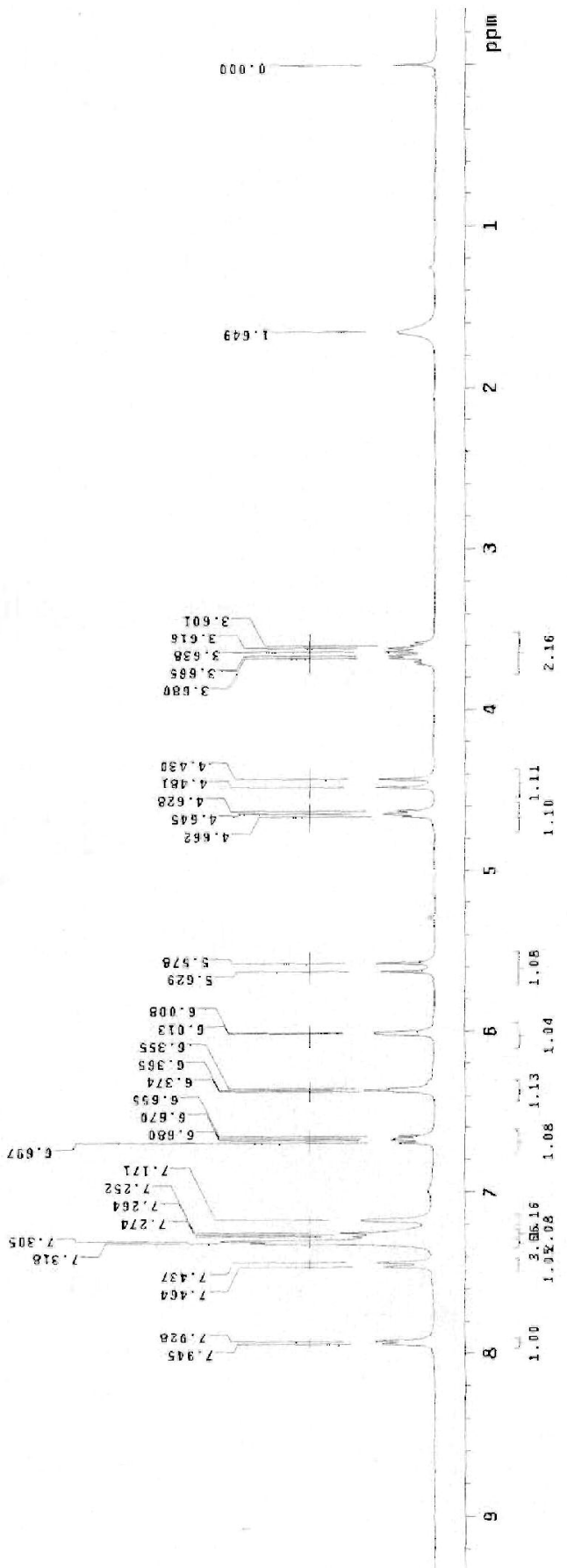

Fig. S-57: $\quad{ }^{1} \mathrm{H} \quad$ Spectra of 5-benzyl-5,6-dihydro-6-hydroxymethylpyrido $[2,3-e]$ pyrrolo[1,2-a]pyrazine 3q. 


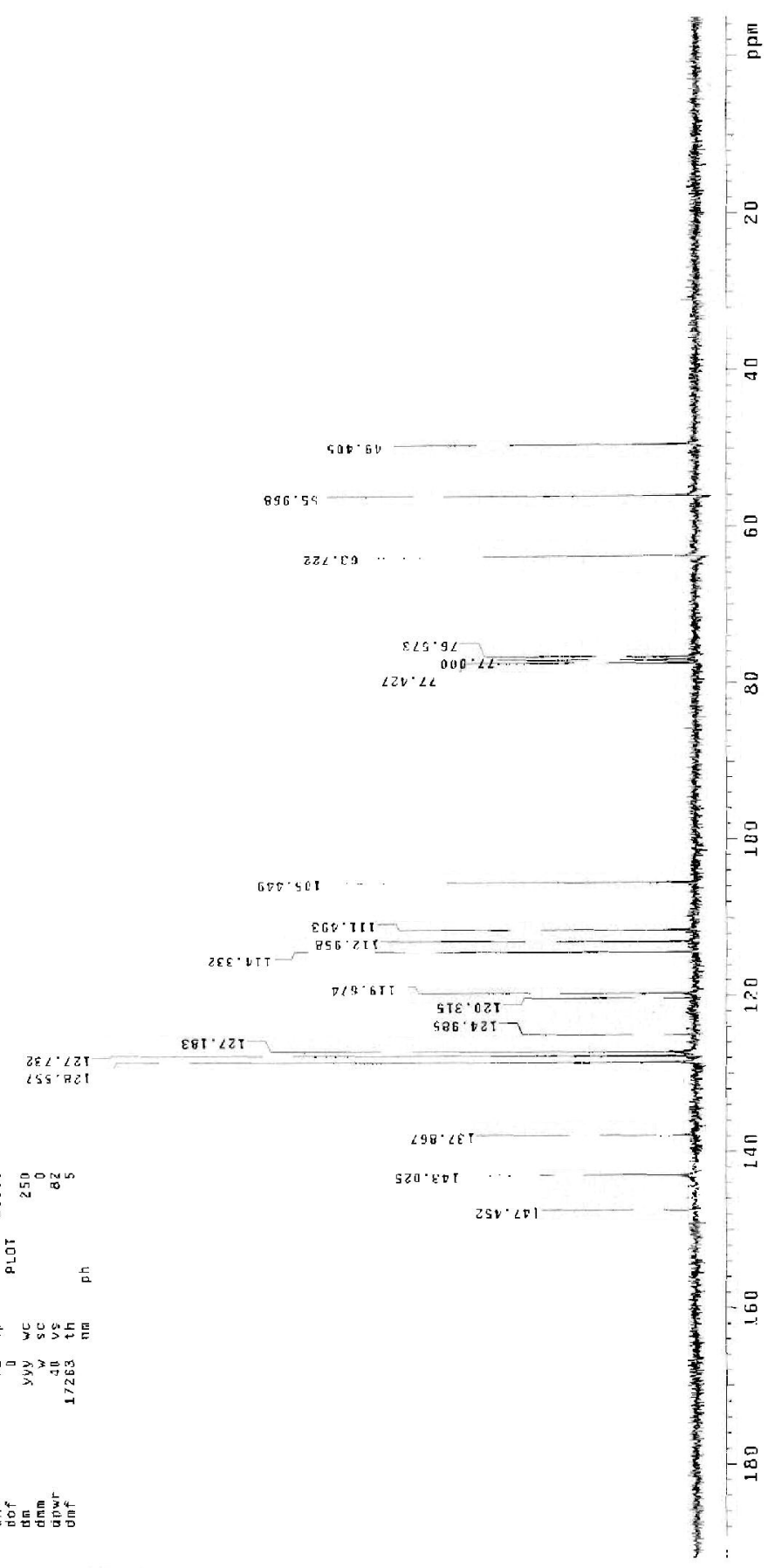

Fig. S-58: ${ }^{13} \mathrm{C}$ Spectra of 5-benzyl-5,6-dihydro-6-hydroxymethylpyrido $[2,3-e]$ pyrrolo $[1,2-a]$ pyrazine $3 q$. 